\title{
Baryon Asymmetry, Neutrino Mixing and \\ Supersymmetric SO(10) Unification
}

\author{
Dissertation \\ zur Erlangung des Doktorgrades \\ des Fachbereichs Physik \\ der Universität Hamburg
}

vorgelegt von

Michael Plümacher

aus Antananarivo

Hamburg

1998 
Gutachter der Dissertation:

Gutachter der Disputation:

Datum der Disputation:

Sprecher des Fachbereichs Physik

und Vorsitzender des

Promotionsausschusses:
Prof. Dr. W. Buchmüller

Prof. Dr. G. Kramer

Prof. Dr. W. Buchmüller

Prof. Dr. J. Bartels

19. Mai 1998

Prof. Dr. B. Kramer 


\begin{abstract}
The baryon asymmetry of the universe can be explained by the out-of-equilibrium decays of heavy right-handed neutrinos. We analyse this mechanism in the framework of a supersymmetric extension of the Standard Model and show that lepton number violating scatterings are indispensable for baryogenesis, even though they may wash-out a generated asymmetry. By assuming a similar pattern of mixings and masses for neutrinos and up-type quarks, as suggested by $\mathrm{SO}(10)$ unification, we can generate the observed baryon asymmetry without any fine tuning, if $(B-L)$ is broken at the unification scale $\Lambda_{\mathrm{GUT}} \sim 10^{16} \mathrm{GeV}$ and, if $m_{\nu_{\mu}} \sim 3 \cdot 10^{-3} \mathrm{eV}$ as preferred by the MSW solution to the solar neutrino deficit.
\end{abstract}

\title{
Zusammenfassung
}

Die Baryonasymmetrie des Universums kann durch den Zerfall schwerer rechtshändiger Neutrinos außerhalb des thermischen Gleichgewichts erklärt werden. Wir untersuchen dies im Rahmen einer supersymmetrischen Erweiterung des Standard-Modells und zeigen, daß leptonzahlverletzende Streuprozesse, die eine erzeugte Asymmetrie wieder vernichten können, für die Baryogenese unverzichtbar sind. Nimmt man für Quarks und Leptonen ähnliche Massen und Mischungswinkel an wie von SO(10)-vereinheitlichten Modellen nahegelegt — so kann man die beobachtete Baryonasymmetrie erzeugen. Dazu wird $(B-L)$ an der Vereinheitlichungsskala $\Lambda_{\mathrm{GUT}} \sim 10^{16} \mathrm{GeV}$ gebrochen, und $m_{\nu_{\mu}} \sim$ $3 \cdot 10^{-3} \mathrm{eV}$ angenommen, wie es die MSW-Lösung des solaren NeutrinoProblems nahelegt. 



\section{Contents}

$\begin{array}{lr}\text { Introduction } & 1\end{array}$

\begin{tabular}{|lll|}
\hline CP Asymmetry in Majorana Neutrino Decays & 4
\end{tabular}

1.1 Self-energy and vertex corrections . . . . . . . . . . . . . . . . . . . 4

1.2 Transition matrix elements $\ldots \ldots \ldots \ldots \ldots \ldots \ldots$

$1.3 \quad$ CP asymmetry in heavy neutrino decays $\ldots \ldots \ldots \ldots$. . . . . . . . . . . 11

$1.4 \quad \mathrm{CP}$ asymmetries in two-body processe $\ldots \ldots \ldots \ldots \ldots \ldots$. . . . . . . . . . 12

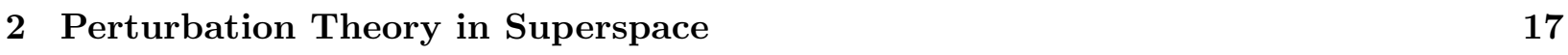

2.1 Superspact . . . . . . . . . . . . . . . . . . . . . 17

2.2 Superfields . . . . . . . . . . . . . . . . . . . . . . . . 19

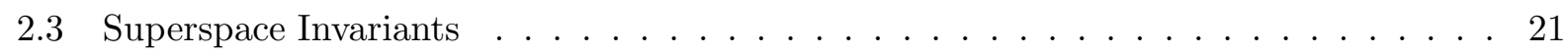

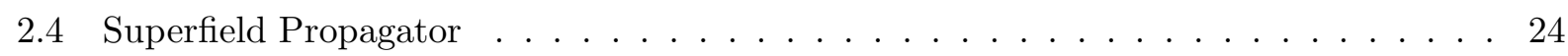

2.5 The Generating Functional $\ldots \ldots \ldots$. . . . . . . . . . . . . . 25

2.6 Feynman Rules in Superspace $\ldots \ldots \ldots \ldots \ldots \ldots$. . . . . . . . . . . 28

\begin{tabular}{|ll|}
3 & Supersymmetric Leptogenesis \\
\hline
\end{tabular}

3.1 The Superpotential $\ldots \ldots \ldots \ldots \ldots$

3.2 The Decay Channels of Heavy Neutrinos $\ldots \ldots \ldots$. . . . . . . . . . . . 33

3.3 Lepton Number Violating Scatterings $\ldots \ldots$. . . . . . . . . . . . . . . . . 40

3.4 Interactions with a Top or a Stop f . . . . . . . . . . . . . . . . . . . . 43

3.5 Neutrino Pair Creation and Annihilation . . . . . . . . . . . . . . . . . . . . . . 45

$3.6 \quad$ MSSM Processes $\ldots \ldots \ldots \ldots \ldots \ldots \ldots$

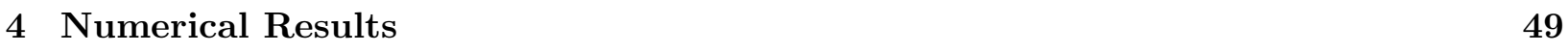

4.1 The Boltzmann Equations $\ldots \ldots \ldots \ldots \ldots$. . . . . . . . . . . . . 49 
4.2 The Generated Lepton Asymmetry $\ldots \ldots \ldots \ldots$. . . . . . . . . . . 52

\begin{tabular}{|ll|l|}
5 & SO(10) Unification and Neutrino Mixing & 57
\end{tabular}

5.1 Neutrino Masses and Mixings $\ldots \ldots \ldots \ldots \ldots$. . . . . . . . . . . 57

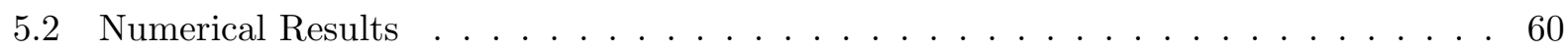

\begin{tabular}{lr}
\hline Conclusions & 65
\end{tabular}

\begin{tabular}{|l|l|}
\hline A One-Loop Integrals & 67 \\
\hline
\end{tabular}

A.1 One-Point Function . . . . . . . . . . . . . . . . . . . . 67

A.2 Two-Point Functions $\ldots \ldots \ldots \ldots \ldots \ldots \ldots$. . . . . . . . . . . . . . . . . .

A.3 Three-Point Functions $\ldots \ldots \ldots \ldots \ldots \ldots$

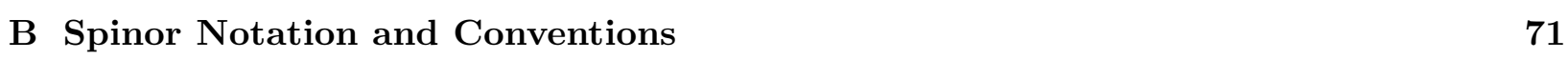

B.1 Weyl Spinors . . . . . . . . . . . . . . . . . . . . . 71

B.2 Four-Component Spinors . . . . . . . . . . . . . . . . . . . 73

B.3 Superfield Products . . . . . . . . . . . . . . . . . . . . 75

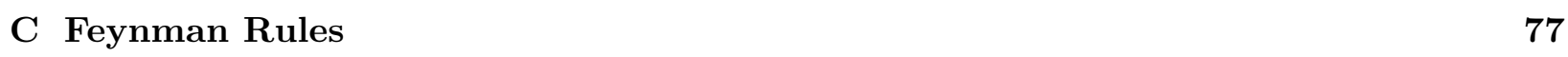

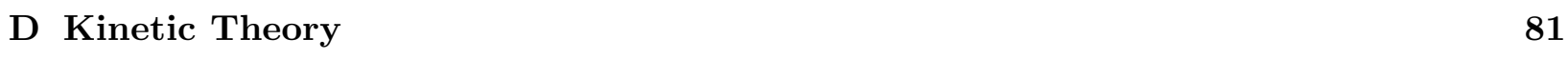

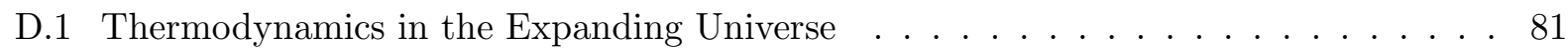

D.2 Boltzmann Equations . . . . . . . . . . . . . . . . 82

\begin{tabular}{|lr|}
\hline E & Reduced Cross Sections \\
\hline
\end{tabular}

E.1 Lepton Number Violating Scatterings $\ldots \ldots \ldots$. . . . . . . . . . . . . . . . 84

E.2 Scattering off a Top or a Stop . . . . . . . . . . . . . . . . . . . . . . . . . . . . . 89

E.3 Neutrino Pair Creation and Annihilation . . . . . . . . . . . . . . . . . . . . . 90

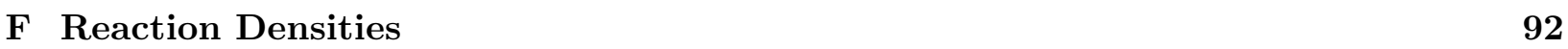

F.1 Bessel functions . . . . . . . . . . . . . . . . . . . . . . . . . 92

F.2 Lepton Number Violating Scatterings $\ldots \ldots \ldots$. . . . . . . . . . . . . . . . 95

F.3 Stop Neutrino Scatterings $\ldots \ldots \ldots$. . . . . . . . . . . . . . . . . . . 97

F.4 Pair Creation and Annihilation of Neutrinos $\ldots \ldots \ldots \ldots$. . . . . . . . . . . . 98 
seeker of truth

follow no path

all paths lead where

truth is here

E. E. Cummings 泪]

\section{Introduction}

The observed baryon asymmetry of the universe is one of the most intriguing problems of particle physics and cosmology. This asymmetry, which is usually expressed as ratio of the baryon density $n_{B}$ to the entropy density $s$ of the universe,

$$
Y_{B}=\frac{n_{B}}{s}=(0.6-1) \cdot 10^{-10},
$$

could in principle be an initial condition of the cosmological evolution. However, this is not compatible with an inflationary phase which seems to be required in a consistent cosmological model [2]. Hence, the baryon asymmetry has to be generated dynamically during the evolution of the universe. This is possible if baryon number is not conserved, if $C$ and $C P$ are violated, and if the universe is not in thermal equilibrium [3].

Although the Standard Model (SM) contains all the necessary ingredients, it is not possible to explain the baryon asymmetry within the SM, i.e. one has to envisage extended theories. Grand unified theories (GUTs) are attractive for various reasons and there have been many attempts to generate $Y_{B}$ at the GUT scale [2]. However, these mechanisms are difficult to reconcile with inflationary scenarios which require reheating temperatures well below the GUT scale.

Preheating, i.e. the non-thermal decay of the oscillating inflaton at the end of inflation via parametric resonance [4], may re-open the window for GUT baryogenesis, since it enables the coherent decay of the inflaton condensate into particles that are more massive than the inflaton itself. How- 
ever, recent calculations indicate that parametric resonance may be ineffective in most inflationary models, if the back reaction of the produced particles onto the condensate, the rescattering of the decay products, and the expansion of the universe are taken into account [5].

In supersymmetric theories, the influence of baryon number carrying scalar condensates along flat directions of the scalar potential, i.e. Affleck-Dine baryogenesis [6], requires further studies, since it is not clear under which conditions this mechanism can generate a baryon asymmetry of the requested magnitude [7].

During the evolution of the early universe, the electroweak phase transition is the last opportunity to generate a baryon asymmetry without being in conflict with the strong experimental bounds on baryon number violation at low energies [8]. However, the thermodynamics of this transition indicates that such scenarios are rather unlikely [9].

Therefore, the baryon asymmetry has to be generated between the reheating scale and the electroweak scale, where baryon plus lepton number $(B+L)$ violating anomalous processes are in thermal equilibrium [10], thereby making a $(B-L)$ violation necessary for baryogenesis. Hence, no asymmetry can be generated within GUT scenarios based on the gauge group SU(5), where $(B-L)$ is a conserved quantity.

Gauge groups containing $\mathrm{SO}(10)$ predict the existence of right-handed neutrinos. In such theories $(B-L)$ is spontaneously broken, one consequence being that the right-handed neutrinos can acquire large Majorana masses, thereby explaining the smallness of the light neutrino masses via the see-saw mechanism [11]. Heavy right-handed Majorana neutrinos violate lepton number in their decays, thus implementing the required $(B-L)$ breaking as lepton number violation. This leptogenesis mechanism was first suggested by Fukugita and Yanagida [12] and has subsequently been studied by several authors (see, e.g., refs. 13 21]). As detailed studies have shown, the observed baryon asymmetry can be generated in non-supersymmetric [13, 14, 15] and supersymmetric theories 16,17.

If one assumes a similar pattern of mass ratios and mixings for leptons and quarks and, if $m_{\nu_{\mu}} \sim 3 \cdot 10^{-3} \mathrm{eV}$ as preferred by the MSW solution to the solar neutrino problem, leptogenesis implies that $(B-L)$ is broken at the unification scale [15]. This suggests a grand unified theory based on the group $\mathrm{SO}(10)$, or one of its extensions, which is directly broken into the standard model gauge group at the unification scale $\sim 10^{16} \mathrm{GeV}$. However, for a successful gauge coupling unification, such a GUT scenario requires low-energy supersymmetry.

Supersymmetric leptogenesis has already been considered in refs. 16, 18, in the approximation that there are no lepton number violating scatterings which can inhibit the generation of a lepton 
number. Another usually neglected problem of leptogenesis scenarios is the necessary production of the right-handed neutrinos after reheating. In non-supersymmetric scenarios one has to assume additional interactions of the right-handed neutrinos for successful leptogenesis [14].

In this thesis, we investigate supersymmetric leptogenesis within the framework of the minimal supersymmetric standard model (MSSM), to which we add right-handed Majorana neutrinos, as suggested by $\mathrm{SO}(10)$ unification [17]. Since $C P$ asymmetries in the decays of these neutrinos are one of the principal ingredients of this model, we start by considering possible sources of $C P$ violation in decays of Majorana neutrinos in the next chapter. In addition to the usually considered one-loop vertex corrections [2], we show how self-energy contributions to the $C P$ asymmetry, which have previously been considered in refs. [18 21], can be consistently taken into account [22]. For simplicity we only consider the non-supersymmetric leptogenesis scenario. However, our results are easily generalized to the supersymmetric case.

In chapter 2 we present superfield techniques, which simplify calculations in theories with exact supersymmetry These techniques are used in chapter 3 where we introduce supersymmetric leptogenesis. In particular, we discuss the neutrino decays and scattering processes that one has to take into account to be consistent [17]. In chapter 0 we develop the full network of Boltzmann equations necessary to get a reliable relation between the input parameters and the final baryon asymmetry. We work out the parameter dependence of the generated baryon asymmetry, and show that by neglecting the lepton number violating scatterings one largely overestimates the generated asymmetry, and that in our scenario the Yukawa interactions are strong enough to produce a thermal population of right-handed neutrinos at high temperatures. Finally, we show in chapter 5 that by assuming a similar pattern of masses and mixings for leptons and quarks one gets the required value for the baryon asymmetry without any fine tuning, provided $(B-L)$ is broken at the GUT scale, and the Dirac mass scale for the neutrinos is of order of the top quark mass, as suggested by $\mathrm{SO}(10)$ unification 15:17.

In appendix $\mathrm{A}$ we summarize some standard formulae for one-loop integrals. In appendix $\mathrm{B}$ we introduce our spinor notation and compile formulae which are needed for the superfield calculations of chapters 2 and 3, while the Feynman rules for component field calculations are presented in appendix Q. After a brief review of thermodynamics in an expanding universe in appendix D, we present the cross sections for the scattering processes discussed in chapter 3 in appendix $\mathbf{E}$. Finally, in appendix $\mathrm{E}$ we discuss some limiting cases in which the corresponding reaction densities can be calculated analytically. 


\section{Chapter 1}

\section{CP Asymmetry in Majorana Neutrino Decays}

In this chapter we study how self-energy diagrams can be consistently taken into account when computing $C P$ asymmetries in heavy particle decays. This is not obvious, since the naive prescription leads to a well-defined result for the $C P$ asymmetry, whereas the individual partial decay widths are infinite.

We investigate this problem in the case of heavy Majorana neutrinos, which are obtained as mass eigenstates if right-handed neutrinos are added to the standard model. Since they are unstable, they cannot appear as in- or out-states of S-matrix elements. Rather, their properties are defined by Smatrix elements for scatterings of stable particles mediated by the unstable neutrino [23]. By using a resummed propagator for the intermediate neutrino, we can separate two-body scattering processes in resonance contributions and remainder. While the $C P$ asymmetries of two-body processes vanish [24], the resonance contributions yield a finite $C P$ asymmetry which can be assigned to the intermediate neutrino

\subsection{Self-energy and vertex corrections}

We consider the standard model with three additional right-handed neutrinos. The corresponding Lagrangian for Yukawa couplings and masses of charged leptons and neutrinos reads

$$
\mathcal{L}_{Y}=\overline{l_{\mathrm{L}}} H \lambda_{l}^{*} e_{\mathrm{R}}+\overline{l_{\mathrm{L}}} \epsilon H^{\dagger} \lambda_{\nu}^{*} \nu_{\mathrm{R}}-\frac{1}{2} \overline{\nu_{\mathrm{R}}^{c}} M \nu_{\mathrm{R}}+\text { h.c. },
$$

where $l_{\mathrm{L}}=\left(\nu_{\mathrm{L}}, e_{\mathrm{L}}\right)$ is the left-handed lepton doublet and $H=\left(H^{+}, H^{0}\right)$ is the standard model Higgs doublet. $\lambda_{l}, \lambda_{\nu}$ and $M$ are $3 \times 3$ complex matrices in the case of three generations. One 
(a)

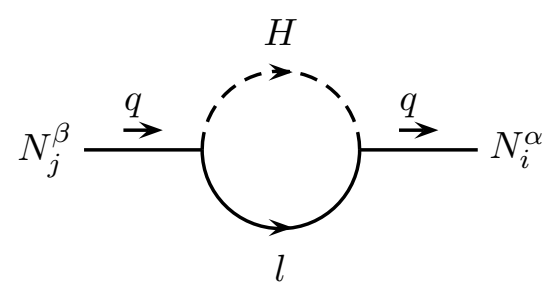

(b)

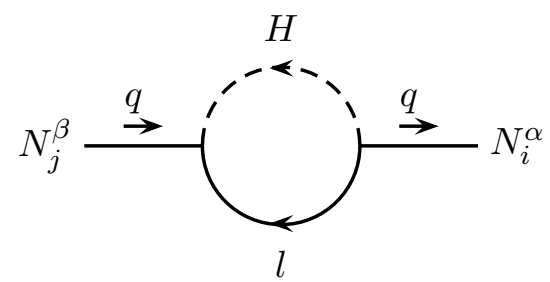

Figure 1.1: Leading order contributions to the self-energy of the heavy Majorana neutrinos. The fermion flow has been chosen parallel to the external momentum $q$.

can always choose a basis for the fields $\nu_{\mathrm{R}}$ such that the mass matrix $M$ is diagonal and real with eigenvalues $M_{i}$. The corresponding physical mass eigenstates are then the three Majorana neutrinos $N_{i}=\nu_{\mathrm{R}_{i}}+\nu_{\mathrm{R}_{i}}^{c}$. At tree level the propagator matrix of these Majorana neutrinos reads

$$
i S_{0}(q)=\frac{i}{q-M+i \epsilon}
$$

This propagator has poles at $q^{2}=M_{i}^{2}$ corresponding to stable particles, whereas the physical Majorana neutrinos are unstable. This is taken into account by summing self-energy diagrams in the usual way, which leads to the resummed propagator

$$
i S(q)=\frac{i}{q-M-\Sigma(q)} .
$$

At one-loop level the two diagrams in fig. 1.1 yield the self energy円

$$
\Sigma_{\alpha \beta}^{i j}(q)=\left(\not P_{\mathrm{R}}\right)_{\alpha \beta} \Sigma_{\mathrm{R}}^{i j}\left(q^{2}\right)+\left(\not q P_{\mathrm{L}}\right)_{\alpha \beta} \Sigma_{\mathrm{L}}^{i j}\left(q^{2}\right)
$$

where $P_{\mathrm{R}, \mathrm{L}}=\frac{1}{2}\left(1 \pm \gamma_{5}\right)$ are the projectors on right- and left-handed chiral states. $\Sigma_{\mathrm{R}}$ and $\Sigma_{\mathrm{L}}$ are the contributions of the diagrams figs. (1.1 a) and (1.1 b), respectively. They can be written as products of a complex function $a\left(q^{2}\right)$ and a hermitian matrix $K$,

$$
\Sigma_{\mathrm{L}}\left(q^{2}\right)=\left(\Sigma_{\mathrm{R}}\left(q^{2}\right)\right)^{T}=a\left(q^{2}\right) K, \quad K=\lambda_{\nu}^{\dagger} \lambda_{\nu}
$$

$a\left(q^{2}\right)$ is given by the usual form factor $B_{0}\left(q^{2}, 0,0\right)$ defined in eq. (A.5), whose finite part reads in the $\overline{M S}$-scheme,

$$
a\left(q^{2}\right)=\frac{1}{16 \pi^{2}}\left(\ln \frac{\left|q^{2}\right|}{\mu^{2}}-2-i \pi \Theta\left(q^{2}\right)\right) .
$$

\footnotetext{
${ }^{1}$ For the calculations we use the non-supersymmetric subset of the Feynman rules in App. Q, by identifying the SM Higgs doublet $H$ with the supersymmetric scalar Higgs doublet $H_{2}$.
} 

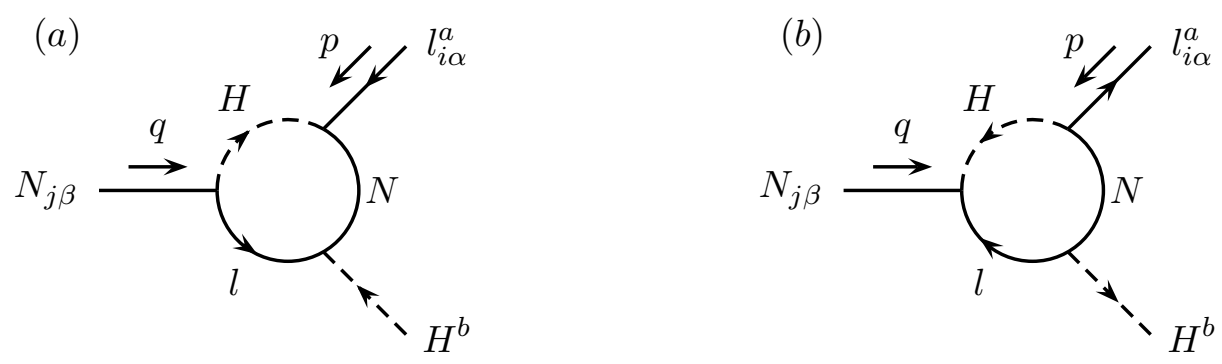

Figure 1.2: One-loop corrections to the couplings of heavy Majorana neutrinos $N_{j}$ to anti-lepton Higgs states (a) and lepton Higgs states (b). The fermion flow has been chosen according to the external lepton lines.

For simplicity we will often omit the argument of $a$ in the following, however one should keep in mind that $a$ depends on $q^{2}$.

According to eqs. (1.3) and (1.4) the resummed propagator $S(q)$ satisfies

$$
\left[q\left(\left(1-\Sigma_{\mathrm{R}}\left(q^{2}\right)\right) P_{\mathrm{R}}+\left(1-\Sigma_{\mathrm{L}}\left(q^{2}\right)\right) P_{\mathrm{L}}\right)-M\right] S(q)=1 .
$$

The fermion propagator $S(q)$ consists of four chiral parts

$$
S(q)=P_{\mathrm{R}} S^{\mathrm{RR}}\left(q^{2}\right)+P_{\mathrm{L}} S^{\mathrm{LL}}\left(q^{2}\right)+P_{\mathrm{L}} \not S^{\mathrm{LR}}\left(q^{2}\right)+P_{\mathrm{R}} \not S^{\mathrm{RL}}\left(q^{2}\right) .
$$

Inserting this decomposition into eq. (1.7), and multiplying the resulting equation from the left and the right with chiral projectors $P_{R, L}$, yields a system of four coupled linear equations for the four parts of the propagator. The solution reads

$$
\begin{aligned}
S^{\mathrm{RR}}\left(q^{2}\right) & =\left[\left(1-\Sigma_{\mathrm{L}}\left(q^{2}\right)\right) \frac{q^{2}}{M}\left(1-\Sigma_{\mathrm{R}}\left(q^{2}\right)\right)-M\right]^{-1}, \\
S^{\mathrm{LR}}\left(q^{2}\right) & =\frac{1}{M}\left(1-\Sigma_{\mathrm{R}}\left(q^{2}\right)\right) S^{\mathrm{RR}}\left(q^{2}\right), \\
S^{\mathrm{LL}}\left(q^{2}\right) & =\left[\left(1-\Sigma_{\mathrm{R}}\left(q^{2}\right)\right) \frac{q^{2}}{M}\left(1-\Sigma_{\mathrm{L}}\left(q^{2}\right)\right)-M\right]^{-1}, \\
S^{\mathrm{RL}}\left(q^{2}\right) & =\frac{1}{M}\left(1-\Sigma_{\mathrm{L}}\left(q^{2}\right)\right) S^{\mathrm{LL}}\left(q^{2}\right) .
\end{aligned}
$$

As discussed below, the diagonal elements of $S(q)$ have approximately the usual Breit-Wigner form.

In addition to the self-energy we need the one-loop vertex function. The two expressions for the coupling of $N$ to $\bar{l}, H^{\dagger}$ (fig. 1.2 a) and $N$ to $l, H$ (fig. 1.2 b) can be written as

$$
\bar{\Gamma}_{\beta \alpha, a b}^{j i}(q, p)=+i \epsilon_{a b}\left[\left(K M b(q, p) \lambda_{\nu}^{T}\right)_{j i} q_{\mu}+\left(K M c(q, p) \lambda_{\nu}^{T}\right)_{j i} p_{\mu}\right]\left(P_{\mathrm{R}} \gamma^{\mu}\right)_{\beta \alpha},
$$




$$
\Gamma_{\alpha \beta, a b}^{i j}(q, p)=-i \epsilon_{a b}\left[\left(\lambda_{\nu}^{*} M b(q, p) K\right)_{i j} q_{\mu}+\left(\lambda_{\nu}^{*} M c(q, p) K\right)_{i j} p_{\mu}\right]\left(P_{\mathrm{R}} \gamma^{\mu}\right)_{\alpha \beta}
$$

Here $b(q, p)$ and $c(q, p)$ are diagonal matrices whose elements are given by the standard three point form factors $C_{0}$ and $C_{12}$ defined in appendix A.3,

$$
\begin{aligned}
b_{k}(q, p) & =\frac{1}{16 \pi^{2}}\left[C_{0}\left(-p-q, q, M_{k}, 0,0\right)+C_{12}\left(-p-q, q, M_{k}, 0,0\right)\right] \\
c_{k}(q, p) & =\frac{1}{16 \pi^{2}}\left[C_{0}\left(-p-q, q, M_{k}, 0,0\right)+2 C_{12}\left(-p-q, q, M_{k}, 0,0\right)\right] .
\end{aligned}
$$

Since we shall only consider amplitudes with massless on-shell leptons, the terms proportional to $c_{k}$ will not contribute. We shall only need the imaginary part of $b_{k}$ which is given by

$$
\operatorname{Im}\left\{b_{k}\left(q^{2}\right)\right\}=\frac{1}{16 \pi \sqrt{q^{2}} M_{k}} f\left(\frac{M_{k}^{2}}{q^{2}}\right) \Theta\left(q^{2}\right),
$$

where the function $f$ is defined as

$$
f(x)=\sqrt{x}\left(1-(1+x) \ln \left(\frac{1+x}{x}\right)\right) .
$$

\subsection{Transition matrix elements}

The two lepton-number violating and the two lepton-number conserving processes are shown in figs. 1.3 $\mathrm{a}-1.3 \mathrm{~d}$. Consider first the contributions of the full propagator, where the full vertices are replaced by tree couplings. The four scattering amplitudes read

$$
\begin{array}{ccc}
\left\langle\bar{l}_{j}^{d}\left(p^{\prime}\right) H^{e \dagger}\left(q-p^{\prime}\right) \mid l_{i}^{a}(p) H^{b}(q-p)\right\rangle= & +i \epsilon_{a b} \epsilon_{d e}\left(\lambda_{\nu}^{T}\right)_{l j}\left(\lambda_{\nu}^{T}\right)_{k i} \\
& \left(C P_{\mathrm{L}} v\left(p^{\prime}\right)\right)^{T} S_{l k}^{\mathrm{LL}}(q)\left(P_{\mathrm{L}} u(p)\right), \\
\left\langle l_{j}^{d}\left(p^{\prime}\right) H^{e}\left(q-p^{\prime}\right) \mid \bar{l}_{i}^{a}(p) H^{b \dagger}(q-p)\right\rangle= & +i \epsilon_{a b} \epsilon_{d e}\left(\lambda_{\nu}^{\dagger}\right)_{l j}\left(\lambda_{\nu}^{\dagger}\right)_{k i} \\
\left\langle l_{j}^{d}\left(p^{\prime}\right) H^{e}\left(q-p^{\prime}\right) \mid l_{i}^{a}(p) H^{b}(q-p)\right\rangle= & -i \epsilon_{a b} \epsilon_{d e}\left(\lambda_{\nu}^{\dagger}\right)_{l j}\left(\lambda_{\nu}^{T}\right)_{k i} \\
& \left.\left(\bar{u}\left(p^{\prime}\right) P_{\mathrm{R}}\right) S_{l k}^{\mathrm{RL}}(q) P_{\mathrm{R}}\right) S_{l k}^{\mathrm{RR}}(q)\left(P_{\mathrm{L}} u(p)\right) \\
\left.\left\langle\bar{l}_{j}^{d}\left(p^{\prime}\right) H^{e \dagger}\left(q-p^{\prime}\right) \mid \bar{l}_{i}^{a}(p) H^{b \dagger}(q-p)\right\rangle\right)^{T} & -i \epsilon_{a b} \epsilon_{d e}\left(\lambda_{\nu}^{T}\right)_{l j}\left(\lambda_{\nu}^{\dagger}\right)_{k i} \\
& \left(C P_{\mathrm{L}} v\left(p^{\prime}\right)\right)^{T} S_{l k}^{\mathrm{LR}}(q) q\left(\bar{v}(p) P_{\mathrm{R}} C\right)^{T}
\end{array}
$$

Here $a, b, d, e$ denote the $\mathrm{SU}(2)$ indices of lepton and Higgs fields and $i, j, k, l$ are generation indices. The relative signs follow from Fermi statistics. 
We are particularly interested in the contributions of a single heavy neutrino to the scattering amplitudes. In order to determine these contributions we have to find the poles and the residues of the propagator matrix. Here an unfamiliar complication arises due to the fact that the self-energy matrix is different for left- and right-handed states. Hence, the different chiral projections of the propagator matrix are diagonalized by different matrices.

$S^{\mathrm{LL}}$ and $S^{\mathrm{RR}}$ are symmetric complex matrices, since $\Sigma_{\mathrm{L}}\left(q^{2}\right)=\left(\Sigma_{\mathrm{R}}\left(q^{2}\right)\right)^{T}$. Hence, $S^{\mathrm{LL}}$ and $S^{\mathrm{RR}}$ can be diagonalized by complex orthogonal matrices $V$ and $U$, respectively,

$$
S^{\mathrm{LL}}\left(q^{2}\right)=V^{T}\left(q^{2}\right) M D\left(q^{2}\right) V\left(q^{2}\right), \quad S^{\mathrm{RR}}\left(q^{2}\right)=U^{T}\left(q^{2}\right) M D\left(q^{2}\right) U\left(q^{2}\right) .
$$

Splitting the self-energy into a diagonal and an off-diagonal part,

$$
\Sigma_{\mathrm{L}}\left(q^{2}\right)=\Sigma_{\mathrm{D}}\left(q^{2}\right)+\Sigma_{\mathrm{N}}\left(q^{2}\right),
$$

one finds

$$
D^{-1}\left(q^{2}\right)=q^{2}\left(1-\Sigma_{\mathrm{D}}\left(q^{2}\right)\right)^{2}-M^{2}+\mathcal{O}\left(\Sigma_{\mathrm{N}}^{2}\right) .
$$

One can easily identify real and imaginary parts of the propagator poles. The pole masses are given by

$$
\bar{M}_{i}^{2}=Z_{M_{i}} M_{i}^{2}, \quad Z_{M_{i}}=1+\frac{K_{i i}}{8 \pi^{2}}\left(\ln \frac{M_{i}^{2}}{\mu^{2}}-2\right),
$$

and the widths are $\Gamma_{i}=K_{i i} M_{i} /(8 \pi)$. In the vicinity of the poles the propagator has the familiar Breit-Wigner form

$$
D_{i}\left(q^{2}\right) \simeq \frac{Z_{i}}{q^{2}-\bar{M}_{i}^{2}+i \bar{M}_{i} \Gamma_{i}}, \quad Z_{i}=1+\frac{K_{i i}}{8 \pi^{2}}\left(\ln \frac{M_{i}^{2}}{\mu^{2}}-1\right) .
$$

We can now easily write down the contribution of a single resonance $N_{l}$ with spin $s$ to the lepton-Higgs scattering amplitudes. Suppressing spin indices for massless fermions, one has

$$
\begin{aligned}
\left\langle\bar{l}_{j}^{d}\left(p^{\prime}\right) H^{e \dagger}\left(q-p^{\prime}\right) \mid l_{i}^{a}(p) H^{b}(q-p)\right\rangle_{l}= & \left\langle\bar{l}_{j}^{d}\left(p^{\prime}\right) H^{\dagger}\left(q-p^{\prime}\right) \mid N_{l}(q, s)\right\rangle \\
& i D_{l}\left(q^{2}\right)\left\langle N_{l}(q, s) \mid l_{i}^{a}(p) H^{b}(q-p)\right\rangle, \\
\left\langle l_{j}^{d}\left(p^{\prime}\right) H^{e}\left(q-p^{\prime}\right) \mid \bar{l}_{i}^{a}(p) H^{b \dagger}(q-p)\right\rangle_{l}= & \left\langle l_{j}^{d}\left(p^{\prime}\right) H^{e}\left(q-p^{\prime}\right) \mid N_{l}(q, s)\right\rangle \\
& i D_{l}\left(q^{2}\right)\left\langle N_{l}(q, s) \mid \bar{l}_{i}^{a}(p) H^{b \dagger}(q-p)\right\rangle, \\
\left\langle l_{j}^{d}\left(p^{\prime}\right) H^{e}\left(q-p^{\prime}\right) \mid l_{i}^{a}(p) H^{b}(q-p)\right\rangle_{l}= & \left\langle l_{j}^{d}\left(p^{\prime}\right) H^{e}\left(q-p^{\prime}\right) \mid N_{l}(q, s)\right\rangle_{L C} \\
& i D_{l}\left(q^{2}\right)\left\langle N_{l}(q, s) \mid l_{i}^{a}(p) H^{b}(q-p)\right\rangle, \\
\left\langle\bar{l}_{j}^{d}\left(p^{\prime}\right) H^{e \dagger}\left(q-p^{\prime}\right) \mid \bar{l}_{i}^{a}(p) H^{b \dagger}(q-p)\right\rangle_{l}= & \left\langle\bar{l}_{j}^{d}\left(p^{\prime}\right) H^{e \dagger}\left(q-p^{\prime}\right) \mid N_{l}(q, s)\right\rangle_{L C} \\
& i D_{l}\left(q^{2}\right)\left\langle N_{l}(q, s) \mid \bar{l}_{i}^{a}(p) H^{b \dagger}(q-p)\right\rangle .
\end{aligned}
$$


(a)

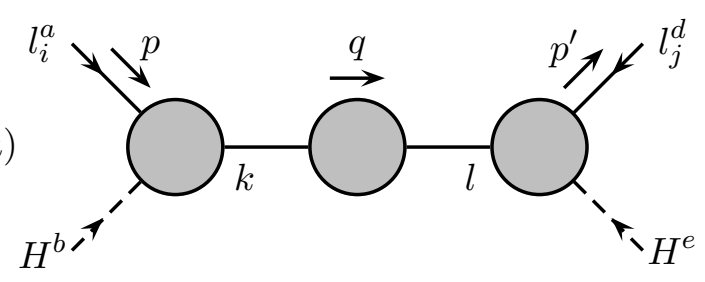

(c)

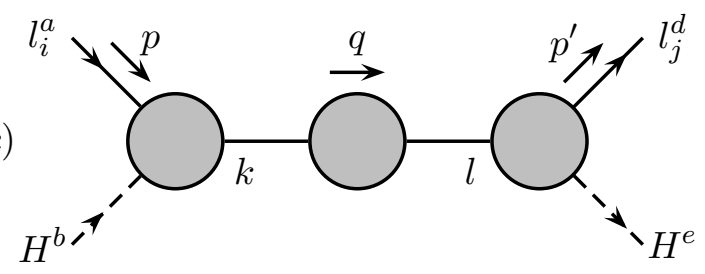

(b)

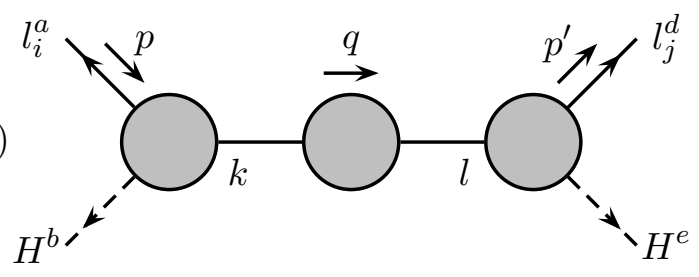

$(d)$

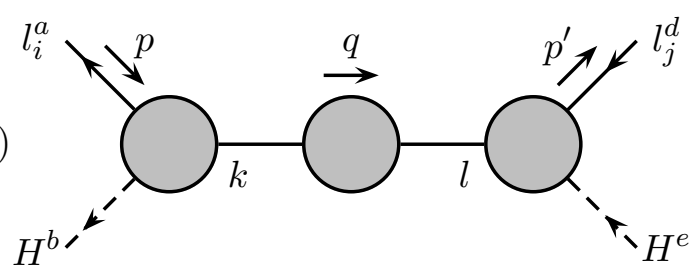

Figure 1.3: s-channel contributions to lepton-Higgs scattering, including full propagators and vertices.

Here the subscript $L C$ distinguishes an amplitude defined by a lepton-number conserving process from the same amplitude defined by a lepton-number violating process. From eqs. (1.9)-(1.12) and (1.23) one finds

$$
\begin{array}{rlrl}
\left\langle N_{l}(q, s) \mid l_{i}^{a}(p) H^{b}(q-p)\right\rangle & = & +i \epsilon_{a b}\left(V\left(q^{2}\right) \lambda_{\nu}^{T}\right)_{l i} \bar{u}_{s}\left(q, M_{l}\right) P_{\mathrm{L}} u(p), \\
\left\langle\bar{l}_{j}^{d}\left(p^{\prime}\right) H^{e \dagger}\left(q-p^{\prime}\right) \mid N_{l}(q, s)\right\rangle= & -i \epsilon_{d e}\left(\lambda_{\nu} V^{T}\left(q^{2}\right)\right)_{j l} \bar{v}_{s}\left(q, M_{l}\right) P_{\mathrm{L}} v\left(p^{\prime}\right), \\
\left\langle N_{l}(q, s) \mid \bar{l}_{i}^{a}(p) H^{b \dagger}(q-p)\right\rangle= & -i \epsilon_{a b}\left(U\left(q^{2}\right) \lambda_{\nu}^{\dagger}\right)_{l i} \bar{v}(p) P_{\mathrm{R}} v_{s}\left(q, M_{l}\right), \\
\left\langle l_{j}^{d}\left(p^{\prime}\right) H^{e}\left(q-p^{\prime}\right) \mid N_{l}(q, s)\right\rangle= & +i \epsilon_{d e}\left(\lambda_{\nu}^{*} U^{T}\left(q^{2}\right)\right)_{j l} \bar{u}\left(p^{\prime}\right) P_{\mathrm{R}} u_{s}\left(q, M_{l}\right), \\
\left\langle l_{j}^{d}\left(p^{\prime}\right) H^{e}\left(q-p^{\prime}\right) \mid N_{l}(q, s)\right\rangle_{L C}= & +i \epsilon_{d e}\left(\lambda_{\nu}^{*} \frac{1}{M}\left(1-\Sigma_{\mathrm{L}}\left(q^{2}\right)\right) V^{T}\left(q^{2}\right) M\right)_{j l} \\
\left\langle\bar{l}_{j}^{d}\left(p^{\prime}\right) H^{e \dagger}\left(q-p^{\prime}\right) \mid N_{l}(q, s)\right\rangle_{L C}= & -i \epsilon_{d e}\left(\lambda_{\nu} \frac{1}{M}\left(1-\Sigma_{\mathrm{R}}\left(q^{2}\right)\right) P^{T} u_{s}\left(q, q_{l}^{2}\right) M\right)_{j l} \\
& & \bar{v}_{s}\left(q, M_{l}\right) P_{\mathrm{L}} v\left(p^{\prime}\right),
\end{array}
$$

where we have used the identity $C \bar{v}_{s}^{T}(p)=u_{s}(p)$.

Eqs. (1.32) and (1.33) describe the coupling of the Majorana field $N$ to the lepton fields $l_{i}$ and the Higgs field $H$, and eqs. (1.34) and (1.35) give the couplings of $N$ to the charge conjugated fields $\bar{l}_{i}$ and $H^{\dagger}$. In the case of $C P$ conservation, one has $\lambda_{\nu_{i j}}=\lambda_{\nu_{i j}}^{*}$, which implies $K=K^{T}$ and therefore

$$
\Sigma_{\mathrm{L}}\left(q^{2}\right)=\Sigma_{\mathrm{R}}\left(q^{2}\right), \quad V\left(q^{2}\right)=U\left(q^{2}\right)
$$


This yields

$$
\left\langle N_{l}(q, s) \mid l_{i}^{a}(p) H^{b}(q-p)\right\rangle=\left\langle N_{l}(\tilde{q}, s) \mid \bar{l}_{i}^{a}(\tilde{p}) H^{b \dagger}(\tilde{q}-\tilde{p})\right\rangle,
$$

with $\tilde{q}=\left(q_{0},-\vec{q}\right), \tilde{p}=\left(p_{0},-\vec{p}\right)$, as required by $C P$ invariance.

The amplitudes given in eqs. (1.32) - (1.35) have been obtained from the lepton-number violating processes figs. 1.3 a and 1.3 b. The lepton-number conserving processes figs. 1.3 $\mathrm{c}$ and 1.3 $\mathrm{d}$ yield the amplitudes given in eqs. (1.36) and (1.37). The consistent definition of an on-shell contribution of a single heavy Majorana neutrino to the two-body scattering amplitudes requires that the transition amplitudes extracted from lepton-number conserving and lepton-number violating processes are consistent. This implies

$$
\begin{aligned}
\left\langle l_{j}^{d}\left(p^{\prime}\right) H^{e}\left(q-p^{\prime}\right) \mid N_{l}(q, s)\right\rangle & =\left\langle l_{j}^{d}\left(p^{\prime}\right) H^{e}\left(q-p^{\prime}\right) \mid N_{l}(q, s)\right\rangle_{L C}, \\
\left\langle\bar{l}_{j}^{d}\left(p^{\prime}\right) H^{e \dagger}\left(q-p^{\prime}\right) \mid N_{l}(q, s)\right\rangle & =\left\langle\bar{l}_{j}^{d}\left(p^{\prime}\right) H^{e \dagger}\left(q-p^{\prime}\right) \mid N_{l}(q, s)\right\rangle_{L C} .
\end{aligned}
$$

From eqs. (1.33), (1.35), (1.36) and (1.37) it is clear that these relations are fulfilled if the mixing matrices $V\left(q^{2}\right)$ and $U\left(q^{2}\right)$ satisfy certain consistency relations. Assuming that the matrix $\lambda_{\nu}$ has an inverse, one reads off

$$
\begin{aligned}
& U_{i j}\left(M_{i}^{2}\right)=\left(M V\left(M_{i}^{2}\right)\left(1-\Sigma_{\mathrm{R}}\left(M_{i}^{2}\right)\right) \frac{1}{M}\right)_{i j}, \\
& V_{i j}\left(M_{i}^{2}\right)=\left(M U\left(M_{i}^{2}\right)\left(1-\Sigma_{\mathrm{L}}\left(M_{i}^{2}\right)\right) \frac{1}{M}\right)_{i j} .
\end{aligned}
$$

The matrices $V$ and $U$ are determined by the requirement that the expressions (cf. eqs. (1.23))

$$
\begin{aligned}
& V\left(q^{2}\right)\left(S^{\mathrm{LL}}\left(q^{2}\right)\right)^{-1} V^{T}\left(q^{2}\right)=V\left(q^{2}\right)\left(\left(1-\Sigma_{\mathrm{R}}\left(q^{2}\right)\right) \frac{q^{2}}{M}\left(1-\Sigma_{\mathrm{L}}\left(q^{2}\right)\right)-M\right) V^{T}\left(q^{2}\right), \\
& U\left(q^{2}\right)\left(S^{\mathrm{RR}}\left(q^{2}\right)\right)^{-1} U^{T}\left(q^{2}\right)=U\left(q^{2}\right)\left(\left(1-\Sigma_{\mathrm{L}}\left(q^{2}\right)\right) \frac{q^{2}}{M}\left(1-\Sigma_{\mathrm{R}}\left(q^{2}\right)\right)-M\right) U^{T}\left(q^{2}\right),
\end{aligned}
$$

are diagonal on-shell, i.e., at $q^{2}=M_{i}^{2}$. Using $\Sigma_{\mathrm{L}}=\Sigma_{\mathrm{D}}+\Sigma_{\mathrm{N}}$, and writing

$$
\begin{aligned}
& V\left(q^{2}\right)=1+v\left(q^{2}\right), \quad v\left(q^{2}\right)=-v^{T}\left(q^{2}\right), \\
& U\left(q^{2}\right)=1+u\left(q^{2}\right), \quad u\left(q^{2}\right)=-u^{T}\left(q^{2}\right),
\end{aligned}
$$

a straightforward calculation yields

$$
\begin{aligned}
& v_{i j}\left(q^{2}\right)=w_{i j}\left(q^{2}\right)\left(M_{i} \Sigma_{\mathrm{N}_{j i}}\left(q^{2}\right)+M_{j} \Sigma_{\mathrm{N}_{i j}}\left(q^{2}\right)\right), \\
& u_{i j}\left(q^{2}\right)=w_{i j}\left(q^{2}\right)\left(M_{i} \Sigma_{\mathrm{N}_{i j}}\left(q^{2}\right)+M_{j} \Sigma_{\mathrm{N}_{j i}}\left(q^{2}\right)\right)
\end{aligned}
$$


where

$$
w_{i j}\left(q^{2}\right)^{-1}=\left(M_{i}-M_{j}\right)\left(1+\frac{M_{i} M_{j}}{q^{2}}\right)-2 a\left(q^{2}\right)\left(M_{i} K_{j j}-M_{j} K_{i i}\right) .
$$

These equations give the matrices $V$ and $U$ to leading order in $\Sigma_{\mathrm{N}}$. They are meaningful as long as the matrix elements of $\Sigma_{\mathrm{N}}$ are small compared to those of $w^{-1}$.

Inserting eqs. (1.48) and (1.49) in eqs. (1.42) and (1.43), one finds that the consistency conditions for the mixing matrices $V$ and $U$ are fulfilled to leading order in $\Sigma_{\mathrm{N}}$. We conclude that the contribution of a single heavy neutrino to two-body scattering processes can indeed be consistently defined. The pole masses are given by eq. (1.26) and the couplings to lepton-Higgs initial and final states are given by eqs. (1.32)-(1.35).

\subsection{CP asymmetry in heavy neutrino decays}

It is now straightforward to evaluate the $C P$ asymmetry in the decay of a heavy Majorana neutrino,

$$
\varepsilon_{i}=\frac{\Gamma\left(N_{i} \rightarrow l H\right)-\Gamma\left(N_{i} \rightarrow \bar{l} H^{\dagger}\right)}{\Gamma\left(N_{i} \rightarrow l H\right)+\Gamma\left(N_{i} \rightarrow \bar{l} H^{\dagger}\right)} .
$$

From eqs. (1.33) and (1.35) one obtains for the partial decay widths, including mixing effects,

$$
\begin{aligned}
\Gamma_{\mathrm{M}}\left(N_{i} \rightarrow \bar{l} H^{\dagger}\right) & \propto \sum_{j}\left|\left(\lambda_{\nu} V^{T}\left(M_{i}^{2}\right)\right)_{j i}\right|^{2} \\
\Gamma_{\mathrm{M}}\left(N_{i} \rightarrow l H\right) & \propto \sum_{j}\left|\left(\lambda_{\nu}^{*} U^{T}\left(M_{i}^{2}\right)\right)_{j i}\right|^{2} .
\end{aligned}
$$

To leading order in $\lambda_{\nu}^{2}$ this yields the asymmetry (cf. eqs. (1.46), (1.47)),

$$
\varepsilon_{i}^{\mathrm{M}}=\frac{1}{K_{i i}} \operatorname{Re}\left\{\left(u\left(M_{i}^{2}\right) K\right)_{i i}-\left(v\left(M_{i}^{2}\right) K^{T}\right)_{i i}\right\}
$$

Using eqs. (1.48) - (1.50) and (1.6), one finally obtains

$$
\varepsilon_{i}^{\mathrm{M}}=-\frac{1}{8 \pi} \sum_{j}\left|w_{i j}\left(M_{i}^{2}\right)\right|^{2}\left(M_{i}^{2}-M_{j}^{2}\right) \frac{M_{j}}{M_{i}} \frac{\operatorname{Im}\left\{K_{\mathrm{N}_{i j}}^{2}\right\}}{K_{i i}} .
$$

Consider first the case where differences between heavy neutrino masses are large, i.e., $\mid M_{i}-$ $M_{j}|\gg| \Gamma_{i}-\Gamma_{j} \mid$. Eq. 1.55) then simplifies to

$$
\varepsilon_{i}^{\mathrm{M}}=-\frac{1}{8 \pi} \sum_{j} \frac{M_{i} M_{j}}{M_{i}^{2}-M_{j}^{2}} \frac{\operatorname{Im}\left\{K_{\mathrm{N}_{i j}}^{2}\right\}}{K_{i i}} .
$$

This is the familiar $C P$ asymmetry due to flavour mixing [18]. It has previously been obtained by considering directly the self-energy correction to the Majorana neutrino decay, without any 

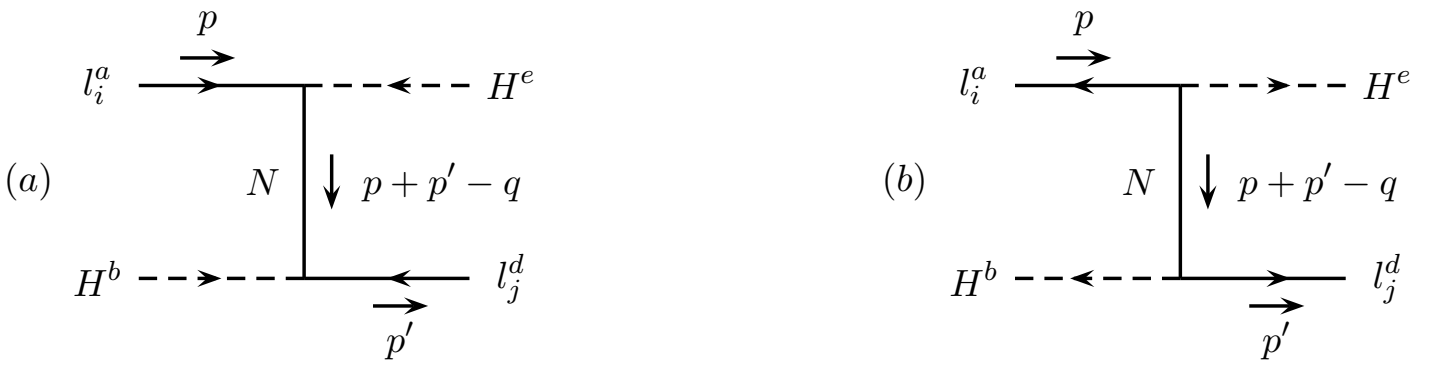

Figure 1.4: u-channel contributions to lepton-Higgs scattering.

resummation. The $C P$ asymmetry $\varepsilon_{i}$ reaches its maximum for $\left|M_{i}-M_{j}\right| \sim\left|\Gamma_{i}-\Gamma_{j}\right|$, where the perturbative expansion breaks down.

Interesting is also the limiting case where the heavy neutrinos become mass degenerate. From eq. (1.55) it is obvious that the $C P$ asymmetry vanishes in this limit. The vanishing of the $C P$ asymmetry for mass degenerate heavy neutrinos is expected on general grounds, since in this case the $C P$ violating phases of the matrix $K$ can be eliminated by a change of basis.

The $C P$ asymmetry due to the vertex corrections is easily obtained using eqs. (1.13), (1.14), (1.17) and (1.18). The partial decay widths corresponding to the full vertex read

$$
\begin{aligned}
\Gamma_{\mathrm{V}}\left(N_{i} \rightarrow \bar{l} H^{\dagger}\right) & \propto \sum_{j}\left|\left(\lambda_{\nu}\left(1-M b K^{T} M\right)\right)_{j i}\right|^{2}, \\
\Gamma_{\mathrm{V}}\left(N_{i} \rightarrow l H\right) & \propto \sum_{j}\left|\left(\lambda_{\nu}^{*}(1-M b K M)\right)_{j i}\right|^{2} .
\end{aligned}
$$

For the corresponding $C P$ asymmetry (1.51) one obtains the familiar result

$$
\varepsilon_{i}^{\mathrm{V}}=-\frac{1}{8 \pi} \sum_{j} \frac{\operatorname{Im}\left\{K_{\mathrm{N}_{i j}}^{2}\right\}}{K_{i i}} f\left(\frac{M_{j}^{2}}{M_{i}^{2}}\right),
$$

where the function $f(x)$ has been defined in eq. (1.18).

\subsection{CP asymmetries in two-body processes}

Let us now consider the $C P$ asymmetries in two-body processes. Here we have to take into account the s-channel amplitudes shown in figs. 1.3 a and 1.3 b, with vertex functions up to one-loop, and the two u-channel amplitudes depicted in figs. 1.4 and 1.4 b. For the u-channel amplitudes vertex and self-energy corrections can be omitted to leading order since the absorptive parts vanish. 
In the following we shall evaluate various contributions to the $C P$ asymmetry

$$
\varepsilon \equiv \frac{\Delta|\mathcal{M}|^{2}}{2|\mathcal{M}|^{2}} \equiv \frac{\left|\mathcal{M}\left(\bar{l} H^{\dagger} \rightarrow l H\right)\right|^{2}-\left|\mathcal{M}\left(l H \rightarrow \bar{l} H^{\dagger}\right)\right|^{2}}{\left|\mathcal{M}\left(\bar{l} H^{\dagger} \rightarrow l H\right)\right|^{2}+\left|\mathcal{M}\left(l H \rightarrow \bar{l} H^{\dagger}\right)\right|^{2}},
$$

where we always sum over generations in initial and final states. There are contributions from the full s-channel propagator, $\Delta|\mathcal{M}|_{s}^{2}$, from the interference between s-channel amplitudes at treelevel and with one-loop vertex corrections, $\Delta|\mathcal{M}|_{s, \Gamma}^{2}$, the interference between tree-level s-channel and u-channel amplitudes, $\Delta|\mathcal{M}|_{s, u}^{2}$, and the interference between s-channel with one-loop vertex corrections and u-channel amplitudes, $\Delta|\mathcal{M}|_{u, \Gamma}^{2}$.

Consider first the $C P$ asymmetry $\varepsilon_{s}$ due to the full propagator. The contribution of a single intermediate neutrino $N_{i}$ is (cf. (1.28), (1.32), (1.33))

$$
\left|\mathcal{M}_{i}\left(l H \rightarrow \bar{l} H^{\dagger}\right)\right|_{s}^{2} \propto\left|D_{i}\left(q^{2}\right)\right|^{2} \sum_{j}\left|\left(V\left(q^{2}\right) \lambda_{\nu}^{T}\right)_{i j}\right|^{2} \sum_{k}\left|\left(\lambda_{\nu} V^{T}\left(q^{2}\right)\right)_{k i}\right|^{2}
$$

Comparison with eq. (1.52) yields immediately

$$
\left|\mathcal{M}_{i}\left(l H \rightarrow \bar{l} H^{\dagger}\right)\right|_{s}^{2} \propto\left|D_{i}\left(q^{2}\right)\right|^{2} \Gamma_{\mathrm{M}}\left(N_{i} \rightarrow \bar{l} H^{\dagger}\right)^{2}
$$

Similarly, one has for the charge conjugated process

$$
\left|\mathcal{M}_{i}\left(\bar{l} H^{\dagger} \rightarrow l H\right)\right|_{s}^{2} \propto\left|D_{i}\left(q^{2}\right)\right|^{2} \Gamma_{\mathrm{M}}\left(N_{i} \rightarrow l H\right)^{2}
$$

The corresponding $C P$ asymmetry is, as expected, twice the asymmetry in the decay due to mixing,

$$
\varepsilon_{s}^{(i)}=\frac{\Delta\left|\mathcal{M}_{i}\right|_{s}^{2}}{2\left|\mathcal{M}_{i}\right|_{s}^{2}} \simeq 2 \varepsilon_{i}^{\mathrm{M}}
$$

It is very instructive to compare the contribution of a single resonance with the $C P$ asymmetry $\varepsilon_{s}$ for the full propagator. Due to the structure of the propagators $S^{\mathrm{LL}}$ and $S^{\mathrm{RR}}$ it is difficult to evaluate $\varepsilon_{s}$ exactly. However, one may easily calculate $\varepsilon_{s}$ perturbatively in powers of $\Sigma_{\mathrm{N}}$, like the mixing matrices $V\left(q^{2}\right)$ and $U\left(q^{2}\right)$ in the previous section.

The full propagator (cf. (1.7)) reads to first order in $\Sigma_{\mathrm{N}}$,

$$
S(q)=S_{\mathrm{D}}(q)+S_{\mathrm{D}}(q) d\left[\Sigma_{\mathrm{N}}^{T}\left(q^{2}\right) P_{\mathrm{R}}+\Sigma_{\mathrm{N}}\left(q^{2}\right) P_{\mathrm{L}}\right] S_{\mathrm{D}}(q)+\ldots
$$

where (cf. 1.25)

$$
S_{\mathrm{D}}(q)=\left[\not\left(1-\Sigma_{\mathrm{D}}\left(q^{2}\right)\right)+M\right] D\left(q^{2}\right) .
$$


It is now straightforward to calculate the matrix elements of the two-body processes, summed over generations in initial and final states,

$$
\begin{aligned}
\left|\mathcal{M}\left(l H \rightarrow \bar{l} H^{\dagger}\right)\right|_{s}^{2}= & 16 p \cdot p^{\prime} q^{2}\left(\frac{1}{2 q^{2}} \operatorname{Tr}\left[K M D\left(q^{2}\right) K^{T} M D^{*}\left(q^{2}\right)\right]+\right. \\
& \operatorname{Re}\left\{\operatorname { T r } \left[K M D\left(q^{2}\right) \Sigma_{\mathrm{N}}^{T}\left(q^{2}\right)\left(1-\Sigma_{\mathrm{D}}\left(q^{2}\right)\right) D\left(q^{2}\right) K^{T} M D^{*}\left(q^{2}\right)+\right.\right. \\
& \left.\left.\left.K D\left(q^{2}\right)\left(1-\Sigma_{\mathrm{D}}\left(q^{2}\right)\right) \Sigma_{\mathrm{N}}\left(q^{2}\right) M D\left(q^{2}\right) K^{T} M D^{*}\left(q^{2}\right)\right]\right\}+\ldots\right) .
\end{aligned}
$$

This yields for the sum and the difference of the $C P$ conjugated matrix elements,

$$
\begin{aligned}
2|\mathcal{M}|_{s}^{2} & =16 p \cdot p^{\prime} \sum_{i, j} A_{i j}+\ldots \\
\Delta|\mathcal{M}|_{s}^{2} & =-16 p \cdot p^{\prime} \sum_{i, j}\left(B_{i j}+C_{i j}\right)+\ldots
\end{aligned}
$$

where

$$
\begin{aligned}
A_{i j} & =\operatorname{Re}\left\{K_{i j}^{2} M_{i} M_{j} D_{j}\left(q^{2}\right) D_{i}^{*}\left(q^{2}\right)\right\}, \\
B_{i j} & =i \operatorname{Im}\left\{K_{\mathrm{N}_{i j}}\right\}^{2} M_{i} M_{j} D_{j}\left(q^{2}\right) D_{i}^{*}\left(q^{2}\right), \\
C_{i j} & =4 q^{2} \operatorname{Re}\left\{i a\left(q^{2}\right) \operatorname{Im}\left\{K_{\mathrm{N}_{i j}}^{2}\right\} M_{i} M_{j}\left(1-\Sigma_{\mathrm{D}}\left(q^{2}\right)_{i}\right) K_{i i} D_{j}\left(q^{2}\right)\left|D_{i}\left(q^{2}\right)\right|^{2}\right\} .
\end{aligned}
$$

For $q^{2} \simeq M_{i}^{2}$ the expressions $A_{i j}$ and $C_{i j}$ are dominated by the contribution of a single resonance $N_{i}$

$$
\begin{aligned}
A_{i i} & \simeq K_{i i}^{2} M_{i}^{2}\left|D_{i}\left(q^{2}\right)\right|^{2}, \\
C_{i j} & \simeq \frac{1}{4 \pi} \operatorname{Im}\left\{K_{\mathrm{N}_{i j}}^{2}\right\} \frac{M_{i}^{3} M_{j}}{M_{i}^{2}-M_{j}^{2}} K_{i i}\left|D_{i}\left(q^{2}\right)\right|^{2} .
\end{aligned}
$$

From eqs. (1.56), (1.73) and (1.74) one reads off that the sum over $C_{i j}$ yields precisely the contribution of the resonance $N_{i}$ to the $C P$ asymmetry,

$$
-\frac{\sum_{j} C_{i j}}{A_{i i}}=-\frac{1}{4 \pi} \sum_{j} \frac{M_{i} M_{j}}{M_{i}^{2}-M_{j}^{2}} \frac{\operatorname{Im}\left\{K_{\mathrm{N}_{i j}}^{2}\right\}}{K_{i i}}=2 \varepsilon_{i}^{\mathrm{M}} .
$$

The second contribution to the $C P$ asymmetry $\varepsilon_{s}$ is due to the sum over $B_{i j}$ (cf. eq. (1.71)). $B_{i j}$ involves two different propagators $(i \neq j)$ and corresponds to an interference term. Using $D_{j}^{*-1}\left(q^{2}\right)=q^{2}-M_{j}^{2}-2 a^{*}\left(q^{2}\right) q^{2} K_{j j}$ and $2 q^{2} \operatorname{Im}\left\{a\left(q^{2}\right)\right\} K_{i i}=-\operatorname{Im}\left\{D_{i}^{-1}\left(q^{2}\right)\right\}$, one can rewrite $C_{i j}$ as follows,

$$
C_{i j}=-i \operatorname{Im}\left\{K_{\mathrm{N}_{i j}}^{2}\right\} M_{i} M_{j} D_{j}^{*-1}\left(q^{2}\right) D_{i}^{-1}\left(q^{2}\right)\left|D_{i}\left(q^{2}\right)\right|^{2}\left|D_{j}\left(q^{2}\right)\right|^{2}
$$


Comparing eqs. (1.71) and (1.76) it is obvious that the sum of both terms, i.e., the $C P$ asymmetry $\varepsilon_{s}$ corresponding to the full propagator, is identically zero! The pole contribution is cancelled by the interference of the pole term with an off-shell propagator.

The contribution to the $C P$ asymmetry $\Delta|\mathcal{M}|_{s, \Gamma}^{2}$ can be computed in a similar manner. The diagrams fig. 1.3 a and 1.3 b yield two contributions for the two vertices. After some algebra one obtains the result (cf. (1.15)

$$
\begin{aligned}
\Delta|\mathcal{M}|_{s, \Gamma}^{2} & =-64 p \cdot p^{\prime} q^{2} \sum_{i, j, k} D_{i j k}+\ldots \\
D_{i j k} & =\operatorname{Im}\left\{K_{i k} K_{j k} K_{i j}\right\} \operatorname{Im}\left\{b_{k}\left(q^{2}\right)\right\} M_{k} M_{j} D_{i}\left(q^{2}\right) D_{j}^{*}\left(q^{2}\right)
\end{aligned}
$$

For $q^{2} \simeq M_{i}^{2}$, one reads off that the sum over $D_{i j k}$ yields, as expected, twice the vertex $C P$ asymmetry,

$$
\varepsilon_{s, \Gamma}\left(M_{i}^{2}\right) \simeq \frac{\sum_{k} D_{i i k}}{A_{i i}}=-\frac{1}{4 \pi} \sum_{k} \frac{\operatorname{Im}\left\{K_{\mathrm{N}_{i k}}^{2}\right\}}{K_{i i}} f\left(\frac{M_{k}^{2}}{M_{i}^{2}}\right)=2 \varepsilon_{i}^{\mathrm{V}} .
$$

A result very similar to eqs. (1.77), (1.78) is obtained for the asymmetry $\Delta|\mathcal{M}|_{s, u}^{2}$, the interference between tree-level s-channel and u-channel amplitudes. One finds $\left(u=\left(q-p-p^{\prime}\right)^{2}\right)$,

$$
\begin{aligned}
\Delta|\mathcal{M}|_{s, u}^{2} & =-32 p \cdot p^{\prime} q^{2} \sum_{i, j, k} E_{i j k}+\ldots \\
E_{i j k} & =\operatorname{Im}\left\{K_{i k} K_{j k} K_{i j}\right\} \operatorname{Im}\left\{a\left(q^{2}\right)\right\} M_{k} M_{j} D_{i}\left(q^{2}\right) D_{j}^{*}\left(q^{2}\right) D_{k}^{*}(u)
\end{aligned}
$$

Integrating the expressions over phase space and using

$$
\int_{-q^{2}}^{0} d u \frac{2 p \cdot p^{\prime}}{u-M_{k}^{2}}=\frac{q^{2} \sqrt{q^{2}}}{M_{k}} f\left(\frac{M_{k}^{2}}{q^{2}}\right)
$$

one finds the cancellation

$$
\int_{-q^{2}}^{0} d u\left(\Delta|\mathcal{M}|_{s, \Gamma}^{2}+\Delta|\mathcal{M}|_{s, u}^{2}\right)=0 .
$$

Finally, we have to consider the $C P$ asymmetry $\Delta|\mathcal{M}|_{u, \Gamma}^{2}$. A straightforward calculation yields

$$
\begin{aligned}
\Delta|\mathcal{M}|_{u, \Gamma}^{2} & =-32 p \cdot p^{\prime} q^{2} \sum_{i, j, k} F_{i j k}+\ldots \\
F_{i j k} & =\operatorname{Im}\left\{K_{i k} K_{j k} K_{j i}\right\} \operatorname{Im}\left\{b_{k}\left(q^{2}\right)\right\} M_{k} M_{j} D_{i}\left(q^{2}\right) D_{j}^{*}(u)
\end{aligned}
$$

After integration over $u$ the resulting matrix $\bar{F}_{i j k}$ is antisymmetric in the indices $j$ and $k$. As a consequence, the asymmetry $\Delta|\mathcal{M}|_{u, \Gamma}^{2}$ is identically zero. 
As we have seen, the total $C P$ asymmetry vanishes to leading order in $\lambda_{\nu}^{2}$. This result has previously been obtained in [24]. It follows from unitarity and $C P T$ invariance. The considered T-matrix elements satisfy the unitarity relation

$$
2 \operatorname{Im}\langle l H|T| l H\rangle=\left\langle l H\left|T^{\dagger} T\right| l H\right\rangle
$$

If, in perturbation theory, the leading contribution to the right-hand side is given by two-particle intermediate states, one has

$$
\sum_{l}\left\langle l H\left|T^{\dagger} T\right| l H\right\rangle=\sum_{l, l^{\prime}}\left(\left|\left\langle l^{\prime} H|T| l H\right\rangle\right|^{2}+\left|\left\langle\overline{l^{\prime}} H^{\dagger}|T| l H\right\rangle\right|^{2}\right)+\ldots
$$

CPT invariance implies

$$
\left\langle l^{\prime} H|T| l H\right\rangle=\left\langle\bar{l} H^{\dagger}|T| \bar{l}^{\prime} H^{\dagger}\right\rangle .
$$

From eqs. (1.86) - (1.88) one then obtains

$$
\sum_{l, l^{\prime}}\left(\left|\left\langle\bar{l}^{\prime} H^{\dagger}|T| l H\right\rangle\right|^{2}-\left|\left\langle l^{\prime} H|T| \bar{l} H^{\dagger}\right\rangle\right|^{2}\right)+\ldots=0 .
$$

In [24] it was concluded that away from resonance poles, where ordinary perturbation theory holds, the $C P$ asymmetry (1.89) vanishes to order $\lambda_{\nu}^{6}$. Corrections due to four-particle intermediate states are $\mathcal{O}\left(\lambda_{\nu}^{8}\right)$. In this paper we have developed a resummed perturbative expansion in powers of $\Sigma_{\mathrm{N}}$ which is also valid for $s \simeq M_{i}^{2}$. The same argument then implies that in this case the $C P$ asymmetry (1.89) vanishes to order $\lambda_{\nu}^{2}$ whith corrections $\mathcal{O}\left(\lambda_{\nu}^{4}\right)$.

The nature of the cancelation is different for different subprocesses. For the full propagator, the $C P$ asymmetry vanishes identically for fixed external momenta. Interference contributions between various s-channel and u-channel amplitudes cancel after phase space integration. In applications at finite temperature the standard practice [2] is to treat in the Boltzmann equations resonance contributions and the remaining two-body cross sections differently. This procedure yields for the $C P$ asymmetry of the decaying heavy neutrino $N_{i}$ the sum of mixing and vertex contribution, $\varepsilon_{i}=\varepsilon_{i}^{\mathrm{M}}+\varepsilon_{i}^{\mathrm{V}}$. 


\section{Chapter 2}

\section{Perturbation Theory in Superspace}

In this chapter we give a short review of the superspace formulation of theories with global supersymmetry. Since in the following chapters we will only encounter chiral superfields, we restrict ourselves to chiral superfields and leave aside the superspace formulation of gauge theories, which can be found e.g. in ref. [25]. In particular, we will present the powerful calculational tool of perturbation theory in superspace, which drastically simplifies calculations of $S$-matrix elements in theories with exact supersymmetry.

\subsection{Superspace}

Supersymmetry transformations are generated by operators $Q$ which transform bosons into fermions, i.e. these generators have fermionic character [26,27]. The operators $Q$ and their Hermitian adjoints $\bar{Q}$ can be chosen to be Weyl spinors币, which obey anticommutation relations

$$
\begin{aligned}
& \left\{Q_{\alpha}, Q_{\beta}\right\}=\left\{\bar{Q}_{\dot{\alpha}}, \bar{Q}_{\dot{\beta}}\right\}=0, \\
& \left\{Q_{\alpha}, \bar{Q}_{\dot{\alpha}}\right\}=2 \sigma_{\alpha \dot{\alpha}}{ }^{\mu} P_{\mu}, \\
& {\left[Q_{\alpha}, P_{\mu}\right]=\left[\bar{Q}_{\dot{\alpha}}, P_{\mu}\right]=0}
\end{aligned}
$$

where $P_{\mu}$ is the energy-momentum operator. Together with the familiar commutation relations for the generators $P_{\mu}$ and $M_{\mu \nu}$ of the Poincaré group, $Q$ and $\bar{Q}$ form a closed algebra, the so-called super-Poincaré algebra.

A compact technique for working out representations of the supersymmetry algebra was proposed by Salam and Strathdee [28]. They introduced Grassmann variables $\theta^{\alpha}(\alpha=1,2)$ and $\bar{\theta}$.

\footnotetext{
${ }^{1}$ For our notations and conventions see app. B.
} 
$(\dot{\alpha}=1,2)$,

$$
\left\{\theta^{\alpha}, \theta^{\beta}\right\}=\left\{\bar{\theta}^{\dot{\alpha}}, \bar{\theta}^{\dot{\beta}}\right\}=\left\{\theta^{\alpha}, \bar{\theta}^{\dot{\beta}}\right\}=0 .
$$

With these anticommuting parameters the supersymmetry algebra can be expressed in terms of commutators,

$$
\begin{aligned}
& {[\theta Q, \theta Q]=[\bar{Q} \bar{\theta}, \bar{Q} \bar{\theta}]=0,} \\
& {[\theta Q, \bar{Q} \bar{\theta}]=2 \theta \sigma^{\mu} \bar{\theta} P_{\mu},} \\
& {\left[\theta Q, P_{\mu}\right]=\left[\bar{Q} \bar{\theta}, P_{\mu}\right]=0 .}
\end{aligned}
$$

Now we are able to exponentiate the super-Poincaré algebra into a group in such a way that the product of two group elements is again a group element. An element of this super-Poincaré group is given by

$$
G(x, \theta, \bar{\theta})=\mathrm{e}^{i(x \cdot P+\theta Q+\bar{\theta} \bar{Q})} .
$$

These group elements generate transformations in the eight-dimensional superspace parametrized by the coordinates $\left(x^{\mu}, \theta^{\alpha}, \bar{\theta}_{\dot{\alpha}}\right)$. In the following we use $z=\left(x^{\mu}, \theta^{\alpha}, \bar{\theta}_{\dot{\alpha}}\right)$ to denote a point in superspace.

Left action of the group element $G(a, \eta, \bar{\eta})$ induces a motion in superspace,

$$
\left(x^{\mu}, \theta, \bar{\theta}\right) \rightarrow\left(x^{\mu}+a^{\mu}+i \eta \sigma^{\mu} \bar{\theta}-i \theta \sigma^{\mu} \bar{\eta}, \theta+\eta, \bar{\theta}+\bar{\eta}\right)
$$

This transformation is realized by the differential operator representation of the algebra

$$
\begin{aligned}
& P_{\mu}=i \partial_{\mu} \\
& Q_{\alpha}=\frac{\partial}{\partial \theta^{\alpha}}+i \sigma_{\alpha \dot{\alpha}}^{\mu} \bar{\theta}^{\dot{\alpha}} \partial_{\mu} \\
& \bar{Q}_{\dot{\alpha}}=-\frac{\partial}{\partial \bar{\theta}^{\dot{\alpha}}}-i \theta^{\alpha} \sigma_{\alpha \dot{\alpha}}{ }^{\mu} \partial_{\mu}
\end{aligned}
$$

where differentiation with respect to spinor parameters $\theta$ and $\bar{\theta}$ is defined by

$$
\frac{\partial}{\partial \theta^{\alpha}} \theta^{\beta} \equiv \delta_{\alpha}^{\beta}, \quad \frac{\partial}{\partial \bar{\theta}^{\dot{\alpha}}} \bar{\theta}^{\dot{\beta}} \equiv \delta_{\dot{\alpha}}^{\dot{\beta}} .
$$

The usual rules for raising and lowering spinor indices (cf. app. B) therefore give an additional sign, if the index position in the differentiations is changed,

$$
\varepsilon^{\alpha \beta} \frac{\partial}{\partial \theta^{\beta}}=-\frac{\partial}{\partial \theta_{\alpha}}, \quad \varepsilon_{\dot{\alpha} \dot{\beta}} \frac{\partial}{\partial \bar{\theta}_{\dot{\beta}}}=-\frac{\partial}{\partial \bar{\theta}^{\dot{\alpha}}} .
$$


Correspondingly, right action of the group elements induces an anti-realization of the superPoincaré group generated by covariant derivatives $D$ and $\bar{D}$,

$$
\begin{aligned}
D_{\alpha} & =\frac{\partial}{\partial \theta^{\alpha}}-i \sigma_{\alpha \dot{\alpha}}{ }^{\mu} \bar{\theta}^{\dot{\alpha}} \partial_{\mu} \\
\bar{D}_{\dot{\alpha}} & =-\frac{\partial}{\partial \bar{\theta}^{\dot{\alpha}}}+i \theta^{\alpha} \sigma_{\alpha \dot{\alpha}}{ }^{\mu} \partial_{\mu},
\end{aligned}
$$

which obey the anticommutation rules

$$
\begin{aligned}
& \left\{D_{\alpha}, D_{\beta}\right\}=\left\{\bar{D}_{\dot{\alpha}}, \bar{D}_{\dot{\beta}}\right\}=0 \\
& \left\{D_{\alpha}, \bar{D}_{\dot{\alpha}}\right\}=2 \sigma_{\alpha \dot{\alpha}}{ }^{\mu} P_{\mu} .
\end{aligned}
$$

Furthermore, one can derive the following useful identities

$$
\begin{aligned}
D^{\alpha} D^{\beta} & =-\frac{1}{2} \varepsilon^{\alpha \beta} D^{2}, \\
\bar{D}^{\dot{\alpha}} \bar{D}^{\dot{\beta}} & =\frac{1}{2} \varepsilon^{\dot{\alpha} \dot{\beta}} \bar{D}^{2}, \\
D^{\alpha} \bar{D}^{2} D_{\alpha} & =\overline{D_{\dot{\alpha}}} D^{2} \bar{D}^{\dot{\alpha}}, \\
\bar{D}^{2} D^{2} \bar{D}^{2} & =-16 \square \bar{D}^{2}, \\
D^{2} \bar{D}^{2} D^{2} & =-16 \square D^{2},
\end{aligned}
$$

where the squared covariant derivatives are given by

$$
\begin{aligned}
D^{2} \equiv D^{\alpha} D_{\alpha} & =-\varepsilon^{\alpha \beta} \frac{\partial}{\partial \theta^{\alpha}} \frac{\partial}{\partial \theta^{\beta}}+2 i\left(\bar{\theta} \bar{\sigma}^{\mu} \frac{\partial}{\partial \theta}\right) \partial_{\mu}+\bar{\theta}^{2} \square \\
\bar{D}^{2} \equiv \bar{D}_{\dot{\alpha}} \bar{D}^{\dot{\alpha}} & =\varepsilon^{\dot{\alpha} \dot{\beta}} \frac{\partial}{\partial \bar{\theta}^{\dot{\alpha}}} \frac{\partial}{\partial \bar{\theta}^{\dot{\beta}}}+2 i\left(\theta \sigma^{\mu} \frac{\partial}{\partial \bar{\theta}}\right) \partial_{\mu}+\theta^{2} \square
\end{aligned}
$$

\subsection{Superfields}

Supersymmetric theories are most easily formulated in terms of superfields in superspace. In order to get a feeling for how to define a superfield, let us first consider an ordinary quantum field $\phi(x)$ which depends only on the coordinates $x^{\mu}$ of Minkowski space. Translations of these coordinates are generated by the operator $P_{\mu}$, and we can consider $\phi(x)$ to have been translated from $x^{\mu}=0$,

$$
\phi(x)=\mathrm{e}^{i x \cdot P} \phi(0) \mathrm{e}^{-i x \cdot P}
$$


In complete analogy, a superfield $F(x, \theta, \bar{\theta})$ can be defined as 27]

$$
F(x, \theta, \bar{\theta})=G(x, \theta, \bar{\theta}) F(0,0,0) G^{-1}(x, \theta, \bar{\theta})
$$

where $G(x, \theta, \bar{\theta})$ is an element of the super-Poincaré group given by eq. (2.8). This means that a superfield is defined as a Taylor expansion in $\theta$ and $\bar{\theta}$ with coefficients which are themselves local fields in Minkowski space. Due to the Grassmann nature of $\theta$ and $\bar{\theta}$, this expansion breaks off, and the most general superfield reads

$$
\begin{aligned}
F(x, \theta, \bar{\theta})= & f(x)+\theta \phi(x)+\bar{\theta} \bar{\chi}(x)+\theta^{2} m(x)+\bar{\theta}^{2} n(x)+\theta \sigma^{\mu} \bar{\theta} v_{\mu}(x) \\
& +\theta^{2} \bar{\theta} \bar{\lambda}(x)+\bar{\theta}^{2} \theta \psi(x)+\theta^{2} \bar{\theta}^{2} d(x) .
\end{aligned}
$$

This superfield contains as Taylor coefficients four complex scalar fields $f, m, n$ and $d$, one complex vector $v_{\mu}$, two spinors $\phi$ and $\psi$ in the $\left(\frac{1}{2}, 0\right)$ representation and two spinors $\bar{\chi}$ and $\bar{\lambda}$ in the $\left(0, \frac{1}{2}\right)$ representation of the Lorentz group, altogether 16 fermionic and 16 bosonic field components.

Consequently, superfields form linear representations of the supersymmetry algebra which are, in general, highly reducible. Irreducible representations can be constructed by imposing constraints on the superfields. Like all covariant derivatives, $D$ and $\bar{D}$ can be used to impose covariant conditions. Chiral superfields $\Phi$ are characterized by the condition

$$
\bar{D}_{\dot{\alpha}} \Phi=0
$$

This first order differential equation is most easily solved in terms of the variables $y^{\mu}=x^{\mu}-i \theta \sigma^{\mu} \bar{\theta}$ and $\theta$ since

$$
\bar{D}_{\dot{\alpha}}\left(x^{\mu}-i \theta \sigma^{\mu} \bar{\theta}\right)=0 \quad \text { and } \quad \bar{D}_{\dot{\alpha}} \theta=0 \text {. }
$$

Then an arbitrary function of $y$ and $\theta$ is a chiral superfield,

$$
\Phi(y, \theta)=A(y)+\sqrt{2} \theta \psi(y)+\theta^{2} F(y) .
$$

This is the most general solution to eq. (2.29), as may be seen by expressing the covariant derivatives in terms of $y, \theta$ and $\bar{\theta}$,

$$
\begin{aligned}
D_{\alpha} & =\frac{\partial}{\partial \theta^{\alpha}}-2 i \sigma_{\alpha \dot{\alpha}}{ }^{\mu} \bar{\theta}^{\dot{\alpha}} \frac{\partial}{\partial y^{\mu}} \\
\bar{D}_{\dot{\alpha}} & =-\frac{\partial}{\partial \bar{\theta}^{\dot{\alpha}}} .
\end{aligned}
$$


By Taylor expansion in $\theta$ and $\bar{\theta}$, we can write a chiral superfield as a function of the original superspace coordinates $x^{\mu}, \theta$ and $\bar{\theta}$,

$$
\begin{aligned}
\Phi(x, \theta, \bar{\theta})= & A(x)-i \theta \sigma^{\mu} \bar{\theta} \partial_{\mu} A(x)-\frac{1}{4} \theta^{2} \bar{\theta}^{2} \square A(x) \\
& +\sqrt{2} \theta \psi(x)+\frac{i}{\sqrt{2}} \theta^{2} \partial_{\mu} \psi(x) \sigma^{\mu} \bar{\theta}+\theta^{2} F(x) .
\end{aligned}
$$

Conjugation gives an antichiral superfield $\bar{\Phi}$ which satisfies the constraint

$$
D_{\alpha} \bar{\Phi}=0
$$

It is a natural function of $\bar{y}^{\mu}=x^{\mu}+i \theta \sigma^{\mu} \bar{\theta}$ and $\bar{\theta}$, and its power series expansio is obtained from eqs. (2.31) and (2.34) by conjugation.

Supersymmetry invariant actions can be constructed from chiral superfields and their products. It is clear from the expansion (2.31) that a product of chiral superfields is again a chiral superfield (cf. app. B.3), whereas a product of a chiral and an antichiral superfield will satisfy neither eq. (2.29) nor eq. 2.35). However, not every component of these product superfields can be used to construct supersymmetric actions. To be able to formulate supersymmetric theories we have to isolate the components which are invariant under supersymmetry transformations, up to total derivatives.

\subsection{Superspace Invariants}

The general method by which a translation invariant action is derived from fields is to integrate a Lagrange density $\mathcal{L}(x)$ over $d^{4} x$. The result is translationally invariant if surface terms vanish. Similarly, SUSY invariant actions can be constructed by integration over superspace, once we have defined an integral over the Grassmann variables $\theta$ and $\bar{\theta}$. This Berezin integral is determined by imposing linearity and translation invariance, except for the normalization which is fixed by the definitions 29]

$$
\int d^{2} \theta \theta^{2}=1 \quad \text { and } \quad \int d^{2} \bar{\theta} \bar{\theta}^{2}=1
$$

with all other integrals vanishing. The two-dimensional volume elements are defined by

$$
\begin{aligned}
& d^{2} \theta=-\frac{1}{4} d \theta^{\alpha} d \theta_{\alpha} \\
& d^{2} \bar{\theta}=-\frac{1}{4} d \bar{\theta} \dot{\alpha} d \bar{\theta}^{\dot{\alpha}} .
\end{aligned}
$$

For integration over superspace we introduce the following integration measures

$$
d^{6} s \equiv d^{4} x d^{2} \theta, \quad d^{6} \bar{s} \equiv d^{4} x d^{2} \bar{\theta},
$$




$$
d^{8} z \equiv d^{4} x d^{2} \theta d^{2} \bar{\theta}
$$

If we adopt the convention of dropping total divergences, i.e. surface integrals, the differential operators $-\frac{1}{4} D^{2}$ and $-\frac{1}{4} \bar{D}^{2}$ are equivalent to $d^{2} \theta$ and $d^{2} \bar{\theta}$ under a space-time volume integral,

$$
\begin{aligned}
\int d^{8} z F(x, \theta, \bar{\theta}) & =\int d^{6} s\left(-\frac{1}{4} \bar{D}^{2}\right) F(x, \theta, \bar{\theta}) \\
& =\int d^{6} \bar{s}\left(-\frac{1}{4} D^{2}\right) F(x, \theta, \bar{\theta}) \\
& =\int d^{4} x \frac{D^{2} \bar{D}^{2}}{16} F(x, \theta, \bar{\theta}) \\
& =\int d^{4} x \frac{\bar{D}^{2} D^{2}}{16} F(x, \theta, \bar{\theta}),
\end{aligned}
$$

where $F$ is an arbitrary function of $x, \theta$ and $\bar{\theta}$, i.e. a superfield. It follows that

$$
\int d^{8} z D_{\alpha} F(x, \theta, \bar{\theta})=\int d^{8} z \bar{D}_{\dot{\alpha}} F(x, \theta, \bar{\theta})=0 .
$$

Hence, we immediately get the following rules of integration by parts

$$
\begin{aligned}
& \int d^{8} z F_{1} D_{\alpha} F_{2}=\mp \int d^{8} z\left(D_{\alpha} F_{1}\right) F_{2}, \\
& \int d^{8} z F_{1} \bar{D}_{\alpha} F_{2}=\mp \int d^{8} z\left(\bar{D}_{\alpha} F_{1}\right) F_{2},
\end{aligned}
$$

where the upper (lower) sign is valid if $F_{1}$ is an even (odd) Grassmann function. Similarly, higher powers of covariant derivatives can be partially integrated by means of the following formulae

$$
\begin{aligned}
\int d^{8} z F_{1}\left(D^{2} F_{2}\right) & =\int d^{8} z\left(D^{2} F_{1}\right) F_{2} \\
\int d^{8} z F_{1}\left(\bar{D}^{2} F_{2}\right) & =\int d^{8} z\left(\bar{D}^{2} F_{1}\right) F_{2} \\
\int d^{8} z F_{1}\left(D^{2} \bar{D}^{2} F_{2}\right) & =\int d^{8} z\left(\bar{D}^{2} D^{2} F_{1}\right) F_{2}, \\
\int d^{8} z F_{1}\left(D^{\alpha} \bar{D}^{2} D_{\alpha} F_{2}\right) & =\mp \int d^{8} z\left(\bar{D}^{2} D^{\alpha} F_{1}\right) D_{\alpha} F_{2}, \\
& =\int d^{8} z\left(D^{\alpha} \bar{D}^{2} D_{\alpha} F_{1}\right) F_{2} .
\end{aligned}
$$

We may also define superspace delta distributions

$$
\begin{gathered}
\delta^{2}\left(\theta-\theta^{\prime}\right) \equiv\left(\theta-\theta^{\prime}\right)^{2}, \quad \delta^{2}\left(\bar{\theta}-\overline{\theta^{\prime}}\right) \equiv\left(\bar{\theta}-\overline{\theta^{\prime}}\right)^{2}, \\
\delta^{8}\left(z-z^{\prime}\right) \equiv \delta^{4}\left(x-x^{\prime}\right) \delta^{2}\left(\theta-\theta^{\prime}\right) \delta^{2}\left(\bar{\theta}-\overline{\theta^{\prime}}\right) .
\end{gathered}
$$


Applying covariant derivatives to these delta functions yields

$$
\begin{aligned}
D_{1}^{2} \delta^{2}\left(\theta_{1}-\theta_{2}\right) & =-4 \exp \left[i\left(\theta_{1}-\theta_{2}\right) \sigma^{\mu} \overline{\theta_{1}} \partial_{1, \mu}\right], \\
{\overline{D_{1}}}^{2} \delta^{2}\left(\overline{\theta_{1}}-\overline{\theta_{2}}\right) & =-4 \exp \left[-i \theta_{1} \sigma^{\mu}\left(\overline{\theta_{1}}-\overline{\theta_{2}}\right) \partial_{1, \mu}\right], \\
{\overline{D_{1}}}^{2} D_{1}^{2} \delta^{2}\left(\theta_{1}-\theta_{2}\right) \delta^{2}\left(\overline{\theta_{1}}-\overline{\theta_{2}}\right) & =16 \exp \left[-i\left(\theta_{1} \sigma^{\mu} \overline{\theta_{1}}+\theta_{2} \sigma^{\mu} \overline{\theta_{2}}-2 \theta_{1} \sigma^{\mu} \overline{\theta_{2}}\right) \partial_{1, \mu}\right], \\
D_{1}^{2}{\overline{D_{1}}}^{2} \delta^{2}\left(\theta_{1}-\theta_{2}\right) \delta^{2}\left(\overline{\theta_{1}}-\overline{\theta_{2}}\right) & =16 \exp \left[i\left(\theta_{1} \sigma^{\mu} \overline{\theta_{1}}+\theta_{2} \sigma^{\mu} \overline{\theta_{2}}-2 \theta_{2} \sigma^{\mu} \overline{\theta_{1}}\right) \partial_{1, \mu}\right],
\end{aligned}
$$

where $D_{1}$ and $\overline{D_{1}}$ act on $x_{1}, \theta_{1}$ and $\overline{\theta_{1}}$. The argument of the derivatives can be changed through the following transfer rules

$$
\begin{aligned}
D_{1}^{\alpha} \delta^{8}\left(z_{1}-z_{2}\right) & =-D_{2}^{\alpha} \delta^{8}\left(z_{1}-z_{2}\right) \\
{\overline{D_{1}}}^{\dot{\alpha}} \delta^{8}\left(z_{1}-z_{2}\right) & =-\bar{D}_{2} \dot{\alpha} \delta^{8}\left(z_{1}-z_{2}\right) \\
D_{1}^{2} \delta^{8}\left(z_{1}-z_{2}\right) & =D_{2}^{2} \delta^{8}\left(z_{1}-z_{2}\right) \\
{\overline{D_{1}}}^{2} \delta^{8}\left(z_{1}-z_{2}\right) & ={\overline{D_{2}}}^{2} \delta^{8}\left(z_{1}-z_{2}\right),
\end{aligned}
$$

and since covariant derivatives with different arguments anticommute we also have

$$
\begin{aligned}
{\overline{D_{1}}}^{2} D_{1}^{2} \delta^{8}\left(z_{1}-z_{2}\right) & ={\overline{D_{1}}}^{2} D_{2}^{2} \delta^{8}\left(z_{1}-z_{2}\right) \\
& =D_{2}^{2}{\overline{D_{1}}}^{2} \delta^{8}\left(z_{1}-z_{2}\right) \\
& =D_{2}^{2}{\overline{D_{2}}}^{2} \delta^{8}\left(z_{1}-z_{2}\right) .
\end{aligned}
$$

Superspace integration can be used to construct invariant actions. Consider first the integral over a chiral superfield. Due to the constraint (2.29), a chiral superfield is independent of $\bar{\theta}$, i.e. $\int d^{2} \bar{\theta}$, and hence the full superspace integral gives zero.

Since for chiral superfields the supersymmetry algebra can be realized as coordinate transformations of the chiral subspace of superspace alone, which has coordinates $y^{\mu}$ and $\theta^{\alpha}$ but not $\bar{\theta} \dot{\alpha}$, the $\int d^{4} x d^{2} \theta$ integral, without the $d^{2} \bar{\theta}$, is already an invariant integral for chiral superfields. Therefore, the most general supersymmetric renormalizable Lagrange density involving only one chiral superfield reads

$$
\mathcal{L}=\int d^{2} \theta d^{2} \bar{\theta} \bar{\Phi} \Phi+\left[\int d^{2} \theta\left(\frac{1}{2} m \Phi^{2}+\frac{1}{3} \lambda \Phi^{3}+g \Phi\right)+\text { h.c. }\right] .
$$

We will omit the tadpole term $g \Phi$ in the following, since it can always be eliminated by field redefinitions. 


\subsection{Superfield Propagator}

In close analogy to the usual perturbation theory one can develop a perturbation theory in superspace [30,25]. Our goal is to compute Green functions for superfields,

$$
G^{(N)}\left(z^{1}, \ldots, z^{r} ; z^{r+1}, \ldots, z^{N}\right)=\left\langle 0\left|\mathrm{~T}\left\{\Phi\left(z^{1}\right) \cdots \Phi\left(z^{r}\right) \bar{\Phi}\left(z^{r+1}\right) \cdots \bar{\Phi}\left(z^{N}\right)\right\}\right| 0\right\rangle
$$

where $z^{i}=\left(x^{i \mu}, \theta^{i^{\alpha}}, \overline{\theta^{i}} \dot{\alpha}\right)$ denotes a point in configuration superspace. Let us start by computing the propagator for a chiral superfield, which is constructed from the free Lagrangian

$$
L_{0}=\int d^{8} z \bar{\Phi} \Phi+\left(\int d^{6} s \frac{1}{2} m \Phi^{2}+\text { h.c. }\right) .
$$

Since the operator $-\bar{D}^{2} D^{2} /(16 \square)$ projects on chiral fields,

$$
-\frac{1}{16} \frac{\bar{D}^{2} D^{2}}{\square} \Phi=\Phi \quad \text { if } \quad \bar{D} \Phi=0,
$$

we can use eqs. (2.41)-(2.44), and rewrite the $d^{6} s$ integration in the mass term of the free Lagrangian into an integration over the whole superspace,

$$
\begin{aligned}
L_{0} & =\int d^{8} z\left\{\bar{\Phi} \Phi+\frac{1}{8} m\left(\Phi \frac{D^{2}}{\square} \Phi+\bar{\Phi} \frac{\bar{D}^{2}}{\square} \bar{\Phi}\right)\right\} \\
& =\int d^{8} z \frac{1}{2}(\Phi, \bar{\Phi}) \mathcal{M}\left(\frac{\Phi}{\Phi}\right)
\end{aligned}
$$

with the matrix

$$
\mathcal{M}=\left(\begin{array}{cc}
\frac{1}{4} \frac{m}{\square} D^{2} & 1 \\
1 & \frac{1}{4} \frac{m}{\square} \bar{D}^{2}
\end{array}\right),
$$

where we have assumed a real mass $m$.

To derive equations of motion we have to define a functional derivative in superspace, where we have to take into account the chirality constraint $\bar{D} \Phi=0$. This constraint is automatically respected by varying in the $y$ basis,

$$
\frac{\delta}{\delta \Phi(y, \theta)} \Phi\left(y^{\prime}, \theta^{\prime}\right)=\delta^{4}\left(y-y^{\prime}\right) \delta^{2}\left(\theta-\theta^{\prime}\right) .
$$

Going back to the variable $x$, the variation under superspace integration reads

$$
\frac{\delta}{\delta \Phi(x, \theta, \bar{\theta})} \int d^{8} z^{\prime} \Phi\left(x^{\prime}, \theta^{\prime}, \overline{\theta^{\prime}}\right) F\left(x^{\prime}, \theta^{\prime}, \overline{\theta^{\prime}}\right)=-\frac{1}{4} \bar{D}^{2} F(x, \theta, \bar{\theta}) .
$$


This leads to the formal definition

$$
\frac{\delta}{\delta \Phi(x, \theta, \bar{\theta})} \Phi\left(x^{\prime}, \theta^{\prime}, \overline{\theta^{\prime}}\right)=-\frac{1}{4} \bar{D}^{2} \delta^{8}\left(z-z^{\prime}\right) .
$$

Variation of the free Lagrangian (2.68) then gives equations of motion,

$$
\frac{1}{4}\left(\begin{array}{cc}
\bar{D}^{2} & 0 \\
0 & D^{2}
\end{array}\right) \mathcal{M}\left(\frac{\Phi}{\Phi}\right)=0 .
$$

With eq. 2.67) this leads to

$$
\begin{aligned}
& m \Phi-\frac{1}{4} \bar{D}^{2} \bar{\Phi}=0 \\
& m \bar{\Phi}-\frac{1}{4} D^{2} \Phi=0 .
\end{aligned}
$$

The propagator is defined as Green function of the operator

$$
\frac{1}{4}\left(\begin{array}{cc}
\bar{D}^{2} & 0 \\
0 & D^{2}
\end{array}\right) \mathcal{M}
$$

i.e. the differential equation defining the two point function $\Delta$ reads

$$
\frac{1}{4}\left(\begin{array}{cc}
\bar{D}^{2} & 0 \\
0 & D^{2}
\end{array}\right) \mathcal{M} \Delta=\left(\begin{array}{cc}
\frac{1}{4} \frac{\bar{D}^{2}}{\square} & 0 \\
0 & \frac{1}{4} \frac{D^{2}}{\square}
\end{array}\right) \delta\left(z-z^{\prime}\right),
$$

where the differential operator on the right-hand side implements the chirality constraint (2.29). Solving this equation, one gets the propagator for a chiral superfield

$$
\Delta\left(z, z^{\prime}\right)=\frac{-1}{\square+m^{2}}\left(\begin{array}{cc}
\frac{m}{4} \bar{D}^{2} & \frac{1}{16} \bar{D}^{2} D^{2} \\
\frac{1}{16} D^{2} \bar{D}^{2} & \frac{m}{4} D^{2}
\end{array}\right) \delta\left(z-z^{\prime}\right),
$$

where $-1 /\left(\square+m^{2}\right)$ is a symbolic notation for the Green function of the Klein-Gordon operator $\square+m^{2}$.

\subsection{The Generating Functional}

The generating functional for free Green functions is given by the vacuum-to-vacuum amplitude in the presence of an external classical chiral source $J$ coupled to a free chiral field $\Phi$,

$$
Z_{0}[J, \bar{J}]=\left\langle 0\left|T \exp \left[i \int d^{8} z(J, \bar{J})\left(\begin{array}{cc}
\frac{1}{4} \frac{D^{2}}{\square} & 0 \\
0 & \frac{1}{4} \frac{\bar{D}^{2}}{\square}
\end{array}\right)\left(\frac{\Phi}{\Phi}\right)\right]\right| 0\right\rangle
$$




$$
=N \int \mathcal{D} \Phi \mathcal{D} \bar{\Phi} \exp \left\{i L_{0}+i \int d^{8} z(J, \bar{J})\left(\begin{array}{cc}
\frac{1}{4} \frac{D^{2}}{\square} & 0 \\
0 & \frac{1}{4} \frac{\bar{D}^{2}}{\square}
\end{array}\right)\left(\frac{\Phi}{\Phi}\right)\right\}
$$

where $L_{0}$ is the free Lagrangian from eq. (2.68), and $N$ is a normalization factor which can be chosen such that $Z_{0}[0,0]=1$. In non-supersymmetric quantum field theories the role of this normalization factor is to take out disconnected vacuum bubbles, which would otherwise contribute to Green functions. Although vacuum diagrams vanish in supersymmetric theories because of the nonrenormalization theorems (cf. section 2.6), the normalization factor does not equal unity when the volume of the system tends to infinity [31].

The oscillatory path integral (2.80) is not well defined, and has to be Wick rotated to Euclidean space to be evaluated unambiguously. The Green functions calculated in Euclidean space then yield Green functions in Minkowski space by analytic continuation. We will write all quantities in Minkowski space with the understanding that they can be justified in Euclidean space.

Performing the functional integral with standard techniques, one gets

$$
\begin{aligned}
& Z_{0}[J, \bar{J}]=\exp \left\{-\frac{i}{2} \int d^{8} z d^{8} z^{\prime}(J(z), \bar{J}(z))\left(\begin{array}{cc}
\frac{1}{4} \frac{D^{2}}{\square} & 0 \\
0 & \frac{1}{4} \frac{\bar{D}^{2}}{\square}
\end{array}\right) \Delta\left(z, z^{\prime}\right) \times\right. \\
&\left.\times\left(\begin{array}{cc}
\frac{1}{4} \frac{D^{\prime 2}}{\square} & 0 \\
0 & \frac{1}{4} \frac{\bar{D}^{\prime}}{\square}
\end{array}\right)\left(\begin{array}{c}
J\left(z^{\prime}\right) \\
\bar{J}\left(z^{\prime}\right)
\end{array}\right)\right\},
\end{aligned}
$$

where $\Delta\left(z, z^{\prime}\right)$ is the chiral superfield propagator (2.78). With eq. (2.67) this can be brought to a familiar form,

$$
Z_{0}[J, \bar{J}]=\exp \left\{-\frac{i}{2} \int d^{4} z d^{4} z^{\prime}(J(z), \bar{J}(z)) \Delta_{\mathrm{GRS}}\left(z, z^{\prime}\right)\left(\begin{array}{c}
J\left(z^{\prime}\right) \\
\bar{J}\left(z^{\prime}\right)
\end{array}\right)\right\}
$$

where $\Delta_{\mathrm{GRS}}\left(z, z^{\prime}\right)$ is the superfield propagator of Grisaru, Roček and Siegel 32],

$$
\Delta_{\mathrm{GRS}}\left(z, z^{\prime}\right)=\frac{-1}{\square+m^{2}}\left(\begin{array}{cc}
-\frac{m}{4} \frac{D^{2}}{\square} & 1 \\
1 & -\frac{m}{4} \frac{\bar{D}^{2}}{\square}
\end{array}\right) \delta\left(z-z^{\prime}\right) .
$$


Since $J$ is a chiral source, its functional derivative is defined like in eq. (2.72). The functional derivative of $Z_{0}$ then reads

$$
\left(\begin{array}{c}
\frac{1}{i} \frac{\delta}{\delta J(z)} \\
\frac{1}{i} \frac{\delta}{\delta \bar{J}(z)}
\end{array}\right) Z_{0}=-\int d^{4} z^{\prime} \Delta\left(x, x^{\prime}\right) \frac{1}{4 \square}\left(\begin{array}{c}
D^{2} J(z) \\
\bar{D}^{2} \bar{J}(z)
\end{array}\right) Z_{0}
$$

With eqs. (2.77) and (2.67) this yields a functional equation for $Z_{0}$,

$$
\frac{1}{4}\left(\begin{array}{cc}
\bar{D}^{2} & 0 \\
0 & D^{2}
\end{array}\right) \mathcal{M}\left(\begin{array}{c}
\frac{1}{i} \frac{\delta}{\delta J(z)} \\
\frac{1}{i} \frac{\delta}{\delta \bar{J}(z)}
\end{array}\right) Z_{0}=\left(\begin{array}{c}
J(z) \\
\bar{J}(z)
\end{array}\right) Z_{0}
$$

This equation can easily be generalized to the interacting case. For the $\Phi^{3}$ theory coupled to an external source the equations of motion read

$$
\frac{1}{4}\left(\begin{array}{cc}
\bar{D}^{2} & 0 \\
0 & D^{2}
\end{array}\right) \mathcal{M}\left(\frac{\Phi}{\Phi}\right)-\lambda\left(\frac{\Phi^{2}}{\Phi^{2}}\right)=\left(\frac{J}{J}\right)
$$

By comparison with the functional equation for $Z_{0}$ (2.85) we can write down the defining equation for the full generating functional $Z[J, \bar{J}]$,

$$
\frac{1}{4}\left(\begin{array}{cc}
\bar{D}^{2} & 0 \\
0 & D^{2}
\end{array}\right) \mathcal{M}\left(\begin{array}{c}
\frac{1}{i} \frac{\delta}{\delta J(z)} \\
\frac{1}{i} \frac{\delta}{\delta \bar{J}(z)}
\end{array}\right) Z=\left\{\left(\begin{array}{c}
J(z) \\
\bar{J}(z)
\end{array}\right)+\lambda\left(\begin{array}{c}
\left(\frac{1}{i} \frac{\delta}{\delta J(z)}\right)^{2} \\
\left(\frac{1}{i} \frac{\delta}{\delta \bar{J}(z)}\right)^{2}
\end{array}\right)\right\}
$$

Using the interaction Lagrangian $\mathcal{L}_{\text {INT }}$

$$
\mathcal{L}_{\mathrm{INT}}=\int d^{2} \theta \frac{1}{3} \lambda \Phi^{3}+\int d^{2} \bar{\theta} \frac{1}{3} \lambda \bar{\Phi}^{3},
$$

the operator on the right-hand side of eq. (2.87) can be rewritten as

$$
\begin{aligned}
& \left(\begin{array}{c}
J(z) \\
\bar{J}(z)
\end{array}\right)+\lambda\left(\begin{array}{c}
\left(\frac{1}{i} \frac{\delta}{\delta J(z)}\right)^{2} \\
\left(\frac{1}{i} \frac{\delta}{\delta \bar{J}(z)}\right)^{2}
\end{array}\right)= \\
& =\mathrm{e}^{i \int d^{4} x^{\prime} \mathcal{L}_{\text {INT }}\left(\frac{1}{i} \frac{\delta}{\delta J}, \frac{1}{i} \frac{\delta}{\delta \bar{J}}\right)}\left(\begin{array}{c}
J(z) \\
\bar{J}(z)
\end{array}\right) \mathrm{e}^{-i \int d^{4} x^{\prime} \mathcal{L}_{\text {INT }}\left(\frac{1}{i} \frac{\delta}{\delta J}, \frac{1}{i} \frac{\delta}{\delta \bar{J}}\right)} .
\end{aligned}
$$


Hence, eq. (2.87) yields

$$
\begin{gathered}
\frac{1}{4}\left(\begin{array}{cc}
\bar{D}^{2} & 0 \\
0 & D^{2}
\end{array}\right) \mathcal{M}\left(\begin{array}{c}
\frac{1}{i} \frac{\delta}{\delta J(z)} \\
\frac{1}{i} \frac{\delta}{\delta \bar{J}(z)}
\end{array}\right) \mathrm{e}^{-i \int d^{4} x^{\prime} \mathcal{L}_{\mathrm{INT}}\left(\frac{1}{i} \frac{\delta}{\delta J}, \frac{1}{i} \frac{\delta}{\delta \bar{J}}\right)} Z= \\
=\left(\begin{array}{c}
J(z) \\
\bar{J}(z)
\end{array}\right) \mathrm{e}^{-i \int d^{4} x^{\prime} \mathcal{L}_{\mathrm{INT}}\left(\frac{1}{i} \frac{\delta}{\delta J}, \frac{1}{i} \frac{\delta}{\delta \bar{J}}\right)} Z
\end{gathered}
$$

By comparison with the functional equation for $Z_{0}(2.85)$, the generating functional $Z$ can be related to the free-field generating functional

$$
Z[J, \bar{J}]=\mathrm{e}^{i \int d^{4} x^{\prime} \mathcal{L}_{\mathrm{INT}}\left(\frac{1}{i} \frac{\delta}{\delta J}, \frac{1}{i} \frac{\delta}{\delta \bar{J}}\right)} Z_{0}[J, \bar{J}] .
$$

This relation, familiar from ordinary quantum field theory, is the starting point of perturbation theory in superspace.

\subsection{Feynman Rules in Superspace}

$N$-point Green functions are obtained from the generating functional by functional derivation,

$$
\begin{aligned}
G^{(N)} & \left(z^{1}, \ldots, z^{r} ; z^{r+1}, \ldots, z^{N}\right) \equiv \\
\equiv & \left.(-i)^{N} \frac{\delta}{\delta J\left(z^{1}\right)} \cdots \frac{\delta}{\delta J\left(z^{r}\right)} \frac{\delta}{\delta \bar{J}\left(z^{r+1}\right)} \cdots \frac{\delta}{\delta \bar{J}\left(z^{N}\right)} Z[J, \bar{J}]\right|_{J=\bar{J}=0} \\
= & (-i)^{N} \frac{\delta}{\delta J\left(z^{1}\right)} \cdots \frac{\delta}{\delta J\left(z^{r}\right)} \frac{\delta}{\delta \bar{J}\left(z^{r+1}\right)} \cdots \frac{\delta}{\delta \bar{J}\left(z^{N}\right)} \times \\
& \times\left.\sum_{n=0}^{\infty} \frac{(i)^{n}}{n !} \prod_{j=1}^{n} \int d^{4} x^{\prime j} \mathcal{L}_{\mathrm{INT}}\left(\frac{1}{i} \frac{\delta}{\delta J}, \frac{1}{i} \frac{\delta}{\delta \bar{J}}\right) Z_{0}[J, \bar{J}]\right|_{J=\bar{J}=0}
\end{aligned}
$$

where we have used eq. (2.91) in the last step. The factors

$$
\int d^{4} x^{\prime j} \mathcal{L}_{\mathrm{INT}}\left(\frac{1}{i} \frac{\delta}{\delta J}, \frac{1}{i} \frac{\delta}{\delta \bar{J}}\right)
$$

generate vertices at the superspace points $z^{\prime j}$, and the functional derivatives $\frac{\delta}{\delta J}$ in $\mathcal{L}_{\text {INT }}$, when acting on $Z_{0}$, generate propagators connecting different vertices. The operators $\frac{\delta}{\delta J\left(z^{i}\right)}$ not in $\mathcal{L}_{\text {INT }}$ generate propagators on external lines, which have to be amputated and replaced by superfields $\Phi\left(z^{i}\right)$ in order to get an effective contribution to the Lagrangian. This leads to the following Feynman rules in configuration superspace 
1. Provide external lines with factors $\Phi(z)$ or $\bar{\Phi}(z)$.

2. Due to the chirality of the functional derivative (cf. eq. (2.72)), one has to include one (two) factors $-\frac{1}{4} \bar{D}^{2}$ acting on internal propagators to each $\Phi^{3}$ vertex with two (three) internal lines. The same applies to $\bar{\Phi}^{3}$ vertices and factors $-\frac{1}{4} D^{2}$.

3. Include a coupling constant $\frac{i}{3} \lambda$ and a superspace integration $\int d^{8} z$ for each vertex.

4. Use the GRS-propagators $i \Delta_{\mathrm{GRS}}(2.83)$ for internal lines.

5. Include the usual symmetry factors.

In the next chapter we will illustrate these Feynman rules by performing several sample calculations. Let us first investigate the general structure of diagrams calculated with these rules.

The $d^{2} \theta^{i} d^{2} \bar{\theta}^{i}$ integrals at each vertex can be done, leaving us with one overall $d^{2} \theta d^{2} \bar{\theta}$ integral and the usual Minkowski space integrals over $d^{4} x^{i}$ for each diagram. To see how this comes about let us follow the $\theta$-integrations around an arbitrary closed loop 1 . It consists of propagators, including factors $\delta^{8}\left(z_{i}-z_{i+1}\right)$ and covariant derivatives acting on them, external superfield factors, and $d^{2} \theta^{i} d^{2} \bar{\theta}^{i}$ integrals. Higher powers of covariant derivatives can be reduced by using the identities (2.19) $-(2.23)$.

Consider now the propagator from one vertex $z^{i}$ to another one $z^{j}$, and integrate by parts using eqs. (2.46)-(2.52) to remove all the covariant derivatives from its $\delta$-function. The original contribution becomes a sum of terms. If there are other propagators connecting $z^{i}$ and $z^{j}$ we can use the relations

$$
\begin{aligned}
& \delta_{i j} \delta_{i j}=0 \\
& \delta_{i j} D^{\alpha} \delta_{i j}=0, \\
& \delta_{i j} D^{2} \delta_{i j}=0, \\
& \delta_{i j} D^{\alpha} \bar{D}^{\dot{\alpha}} \delta_{i j}=0, \\
& \delta_{i j} D^{\alpha} \bar{D}^{2} \delta_{i j}=0, \\
& \delta_{i j} D^{2} \bar{D}^{2} \delta_{i j}=\delta_{i j} \bar{D}^{2} D^{2} \delta_{i j}=\delta_{i j} D^{\alpha} \bar{D}^{2} D_{\alpha} \delta_{i j}=\delta_{i j} \bar{D}_{\dot{\alpha}} D^{2} \bar{D}^{\dot{\alpha}} \delta_{i j}=16 \delta_{i j} \delta^{4}\left(x_{i}-x_{j}\right), \\
& \delta_{i j} D^{\alpha} \bar{D}^{2} D^{\beta} \delta_{i j}=-8 \varepsilon^{\alpha \beta} \delta_{i j} \delta^{4}\left(x_{i}-x_{j}\right), \\
& \delta_{i j} \bar{D}^{\dot{\alpha}} D^{2} \bar{D}^{\dot{\beta}} \delta_{i j}=8 \varepsilon^{\dot{\alpha} \dot{\beta}} \delta_{i j} \delta^{4}\left(x_{i}-x_{j}\right),
\end{aligned}
$$

\footnotetext{
${ }^{2}$ We will assume that loop divergences have been properly regularized.
} 
where $\delta_{i j} \equiv \delta^{8}\left(z_{i}-z_{j}\right)$. Hence, the terms generated by the partial integration vanish, unless each of the other $\delta$-functions has exactly two $D$ 's and two $\bar{D}$ 's acting on it. Now the free $\delta$-function can be used to perform the $d^{2} \theta^{j} d^{2} \bar{\theta}^{j}$ integral and shrink all the propagators between $z^{i}$ and $z^{j}$ to a point in $\theta$-space. This procedure can be repeated, until we have removed all $\delta$-functions and performed all $\theta$-integrals except the original one at $z^{i}$. We are left with a sum of terms, all with a single $d^{2} \theta d^{2} \bar{\theta}$ integral, various d'Alembert operators from eqs. (2.22) and (2.23), as well as covariant derivatives acting on the external superfields.

Hence, we have ended up with a $d^{2} \theta d^{2} \bar{\theta}$ integral, even though in the original Lagrangian we may have had chiral $d^{2} \theta$ integrals. This is the perturbative no-renormalization theorem for chiral superfields [33]: radiative corrections do not induce renormalizations of F-terms, i.e. purely chiral mass or interaction terms. Furthermore, all vacuum diagrams vanish, since the $d^{2} \theta d^{2} \bar{\theta}$ integral without any external superfield vanishes. 


\section{Chapter 3}

\section{Supersymmetric Leptogenesis}

In this chapter, which is based on ref. [17], we present the supersymmetric generalization of the leptogenesis scenario suggested by Fukugita and Yanagida [12]. After having introduced the superpotential, we compute all the relevant decay widths, $C P$ asymmetries and scattering cross sections. In order to check the results we have performed two independent calculations. First by using the component field Feynman rules from appendix C, and then by using the superfield techniques introduced in the last chapter.

\subsection{The Superpotential}

In supersymmetric unification scenarios based on $\mathrm{SO}(10)$, the effective theory below the $(B-$ $L$ ) breaking scale is the MSSM supplemented by right-handed Majorana neutrinos. Neglecting soft breaking terms, the masses and Yukawa couplings relevant for leptogenesis are given by the superpotential

$$
\mathcal{W}=\frac{1}{2} N^{c} M N^{c}+\mu H_{1} \epsilon H_{2}+H_{1} \epsilon Q \lambda_{d} D^{c}+H_{1} \epsilon L \lambda_{l} E^{c}+H_{2} \epsilon Q \lambda_{u} U^{c}+H_{2} \epsilon L \lambda_{\nu} N^{c},
$$

where we have chosen a basis in which the Majorana mass matrix $M$ and the Yukawa coupling matrices $\lambda_{d}$ and $\lambda_{l}$ for the down-type quarks and the charged leptons are diagonal with real and positive eigenvalues. The corresponding Lagrange density reads

$$
\mathcal{L}=\int d^{2} \theta \mathcal{W}+\int d^{2} \bar{\theta} \overline{\mathcal{W}}
$$

The chiral superfields in the superpotential (3.1) are most conveniently parametrized in the $y$-basis (cf. section 2.2).

$$
H_{i}(y, \theta)=H_{i}(y)+\sqrt{2} \theta \widetilde{H}_{i}(y)+\theta^{2} F_{H_{i}}(y)
$$




$$
\begin{aligned}
Q(y, \theta) & =\widetilde{q}(y)+\sqrt{2} \theta q_{\mathrm{L}}(y)+\theta^{2} F_{Q}(y), \\
L(y, \theta) & =\widetilde{l}(y)+\sqrt{2} \theta l_{\mathrm{L}}(y)+\theta^{2} F_{l}(y), \\
U^{c}(y, \theta) & =\widetilde{U^{c}}(y)+\sqrt{2} \theta u_{\mathrm{R}}^{c}(y)+\theta^{2} F_{U^{c}}(y), \\
D^{c}(y, \theta) & =\widetilde{D^{c}}(y)+\sqrt{2} \theta d_{\mathrm{R}}^{c}(y)+\theta^{2} F_{D^{c}}(y), \\
E^{c}(y, \theta) & =\widetilde{E^{c}}(y)+\sqrt{2} \theta e_{\mathrm{R}}^{c}(y)+\theta^{2} F_{E^{c}}(y), \\
N^{c}(y, \theta) & =\widetilde{N^{c}}(y)+\sqrt{2} \theta \nu_{\mathrm{R}}^{c}(y)+\theta^{2} F_{N^{c}}(y) .
\end{aligned}
$$

$Q$ and $L$ stand for the left-handed quark and lepton doublets, $U^{c}, D^{c}, E^{c}$ and $N^{c}$ are the righthanded singlet fields, and $H_{i}$ denotes the two Higgs-doublets,

$$
H_{1}=\left(\begin{array}{c}
H_{1}^{0} \\
-H_{1}^{-}
\end{array}\right) \quad \text { and } \quad H_{2}=\left(\begin{array}{c}
H_{2}^{+} \\
H_{2}^{0}
\end{array}\right) .
$$

Besides the usual bispinors for quarks and charged leptons we can introduce Majorana-spinors for the right- and left-handed neutrinos,

$$
N=\left(\begin{array}{c}
\nu_{\mathrm{R}}{ }^{c} \alpha \\
{\overline{\nu_{\mathrm{R}}}}^{c} \dot{\alpha}
\end{array}\right) \quad \text { and } \quad \nu=\left(\begin{array}{c}
\nu_{\mathrm{L} \alpha} \\
\frac{\nu_{\mathrm{L}}}{\alpha}
\end{array}\right) .
$$

In the symmetric phase of the MSSM no mixing occurs between the fermionic partners of gauge and Higgs bosons. Therefore, we have two Dirac higgsinos

$$
\widetilde{h^{0}}=\left(\begin{array}{c}
\widetilde{H_{1}^{0}} \\
\underset{\widetilde{H_{2}^{0}}}{\dot{\alpha}}
\end{array}\right) \quad \text { and } \quad \widetilde{h^{-}}=\left(\begin{array}{c}
\widetilde{H_{1}^{-}} \alpha \\
\widetilde{\widetilde{H_{2}^{+}}} \dot{\alpha}
\end{array}\right) \text {, }
$$

which again form an isospin doublet,

$$
\widetilde{h}=\left(\begin{array}{c}
\widetilde{h^{0}} \\
\widetilde{h^{-}}
\end{array}\right)
$$

The auxiliary component fields of the chiral superfields (3.3)- 3.9) are obtained from the Lagrange density 34,

$$
F_{i}=-\left(\frac{\partial \mathcal{L}}{\partial F_{i}}\right)^{\dagger}
$$

The superpotential (3.1) then yields the following contributions to the auxiliary fields

$$
F_{H_{1}}=\left[\mu H_{2}^{\dagger}+\left(\widetilde{q} \lambda_{d} \widetilde{D^{c}}\right)^{\dagger}+\left(\widetilde{l} \lambda_{l} \widetilde{E^{c}}\right)^{\dagger}\right] \epsilon,
$$




$$
\begin{aligned}
& F_{H_{2}}=\left[-\mu H_{1}^{\dagger}+\left(\widetilde{q} \lambda_{u} \widetilde{U^{c}}\right)^{\dagger}+\left(\widetilde{l} \lambda_{\nu} \widetilde{N^{c}}\right)^{\dagger}\right] \epsilon, \\
& F_{Q_{i}}=\epsilon\left[H_{2}^{\dagger} \widetilde{U_{j}^{c}}\left(\lambda_{u}^{\dagger}\right)_{j i}+H_{1}^{\dagger}{\widetilde{D_{j}^{c}}}^{\dagger}\left(\lambda_{d}^{\dagger}\right)_{j i}\right], \\
& F_{L_{i}}=\epsilon\left[H_{2}^{\dagger}{\widetilde{N_{j}^{c}}}^{\dagger}\left(\lambda_{\nu}^{\dagger}\right)_{j i}+H_{1}^{\dagger}{\widetilde{E_{j}^{c}}}^{\dagger}\left(\lambda_{l}^{\dagger}\right)_{j i}\right], \\
& F_{U_{i}^{c}}=\left(\lambda_{u}^{\dagger}\right)_{i j}{\widetilde{q_{j}}}^{\dagger} \epsilon H_{2}^{\dagger}, \\
& F_{D_{i}^{c}}=\left(\lambda_{d}^{\dagger}\right)_{i j}{\widetilde{q_{j}}}^{\dagger} \epsilon H_{1}^{\dagger}, \\
& F_{E_{i}^{c}}=\left(\lambda_{l}^{\dagger}\right)_{i j}{\widetilde{l_{j}}}^{\dagger} \epsilon H_{1}^{\dagger}, \\
& F_{N_{i}^{c}}=-M_{i} \widetilde{N_{i}^{c}} \widetilde{C}^{\dagger}+\left(\lambda_{\nu}^{\dagger}\right)_{i j}{\widetilde{l_{j}}}^{\dagger} \epsilon H_{2}^{\dagger} .
\end{aligned}
$$

The vacuum expectation values of the neutral Higgs fields generate Dirac masses for the downtype quarks and the charged leptons

$$
v_{1}=\left\langle H_{1}^{0}\right\rangle \neq 0 \quad \Rightarrow \quad m_{d}=\lambda_{d} v_{1} \quad \text { and } \quad m_{l}=\lambda_{l} v_{1}
$$

and for the up-type quarks and the neutrinos

$$
v_{2}=\left\langle H_{2}^{0}\right\rangle \neq 0 \quad \Rightarrow \quad m_{u}=\lambda_{u} v_{2} \quad \text { and } \quad m_{D}=\lambda_{\nu} v_{2}
$$

The Majorana masses $M$ for the right-handed neutrinos, which have to be much larger than the Dirac masses $m_{D}$, offer a natural explanation for the smallness of the light neutrino masses via the see-saw mechanism [1].

To generate a non-vanishing baryon asymmetry, one needs a hierarchy in the Majorana mass matrix $M$. Then the scale at which the asymmetry is generated is given by the mass $M_{1}$ of the lightest right-handed neutrino. Hence, it is convenient to write all the masses and energies in units of $M_{1}$,

$$
a_{j}=\left(\frac{M_{j}}{M_{1}}\right)^{2}, \quad x=\frac{s}{M_{1}^{2}} \quad \text { and } \quad z=\frac{M_{1}}{T},
$$

where $M_{j}$ are the masses of the heavier right-handed neutrinos, $s$ is the squared centre of mass energy of a scattering process and $T$ is the temperature.

\subsection{The Decay Channels of Heavy Neutrinos}

Since at these energy scales supersymmetry breaking terms can be safely neglected, we are working in a theory with exact supersymmetry, i.e. we can use the superfield techniques exposed in the 


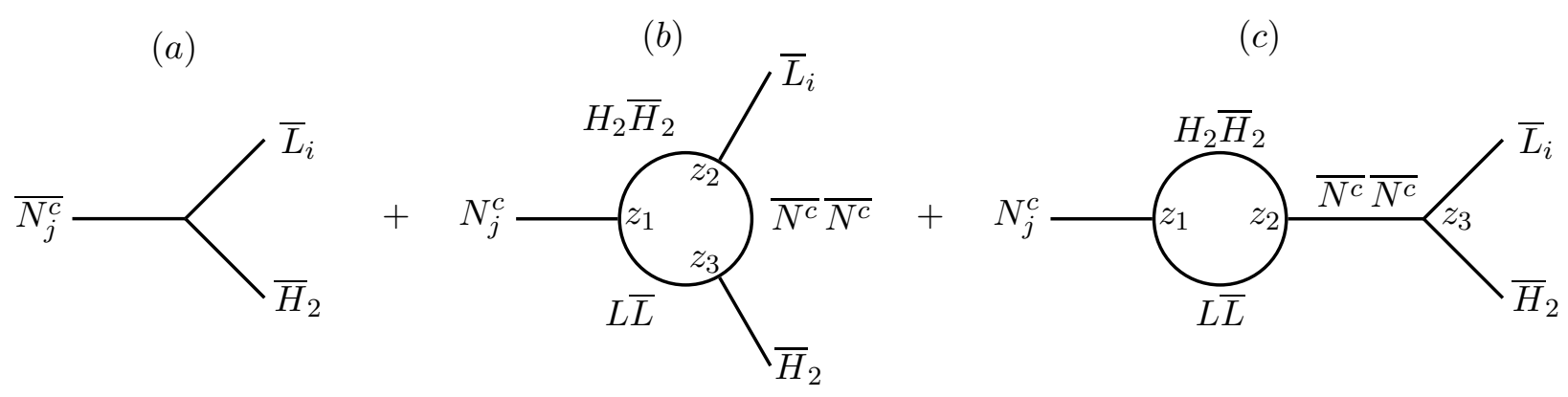

Figure 3.1: Decay modes of the right-handed neutrino superfield.

last chapter to compute decay and scattering amplitudes. To leading order, the decay modes of the right-handed neutrino and its scalar partner with positive lepton number in the final state are all contained in the superfield diagram fig. 3.1 a, corresponding to the superpotential term $-\overline{N^{c}} \lambda_{\nu}^{\dagger} \bar{L} \epsilon \bar{H}_{2}$. Choosing the usual decomposition of the $S$-matrix

$$
S=1+i T
$$

we get the following tree level contribution to the $T$-matrix

$$
T_{N}^{(a)}=-\int d^{4} x \int d^{2} \bar{\theta} \overline{N^{c}} \lambda_{\nu}^{\dagger} \bar{L} \epsilon \bar{H}_{2} .
$$

The decay amplitudes we are interested in are then given by the matrix elements of $i T_{N}^{(a)}$. Let us first concentrate on two-particle final states. According to the component field decompositions (3.3) -(3.9) of the chiral superfields, the right-handed Majorana neutrinos $N_{j}$ can decay into a lepton and a Higgs-boson or into a slepton and a higgsino, while their scalar partners $\widetilde{N_{j}^{c}}$ can decay into a lepton and a higgsino or into a slepton and a Higgs boson (cf. fig. 3.2). The decay widths at tree level read 18

$$
\begin{aligned}
\frac{1}{4} \Gamma_{N_{j}} & :=\Gamma\left(N_{j} \rightarrow \widetilde{l}+\overline{\widetilde{h}}\right)=\Gamma\left(N_{j} \rightarrow \widetilde{l}^{\dagger}+\widetilde{h}\right) \\
& =\Gamma\left(N_{j} \rightarrow l+H_{2}\right)=\Gamma\left(N_{j} \rightarrow \bar{l}+H_{2}^{\dagger}\right)=\frac{M_{j}}{16 \pi} \frac{\left(m_{D}^{\dagger} m_{D}\right)_{j j}}{v_{2}^{2}}, \\
\frac{1}{2} \Gamma_{N_{j}^{c}}^{(2)} & :=\Gamma\left(\widetilde{N_{j}^{c}} \rightarrow \widetilde{l}+H_{2}\right)=\Gamma\left(\widetilde{N_{j}^{c}} \rightarrow \bar{l}+\widetilde{h}\right) \\
& =\Gamma\left({\widetilde{N_{j}^{c}}}^{\dagger} \rightarrow \widetilde{l}^{\dagger}+H_{2}^{\dagger}\right)=\Gamma\left({\widetilde{N_{j}^{c}}}^{\dagger} \rightarrow l+\overline{\widetilde{h}}\right)=\frac{M_{j}}{8 \pi} \frac{\left(m_{D}^{\dagger} m_{D}\right)_{j j}}{v_{2}^{2}} .
\end{aligned}
$$

According to eq. (D.15), the reaction densities for these decays are then given by

$$
\gamma_{N_{j}}=2 \gamma_{\overline{N_{j}^{c}}}^{(2)}=\frac{M_{1}^{4}}{4 \pi^{3}} \frac{\left(m_{D}^{\dagger} m_{D}\right)_{j j}}{v_{2}^{2}} \frac{a_{j} \sqrt{a_{j}}}{z} \mathrm{~K}_{1}\left(z \sqrt{a_{j}}\right) .
$$



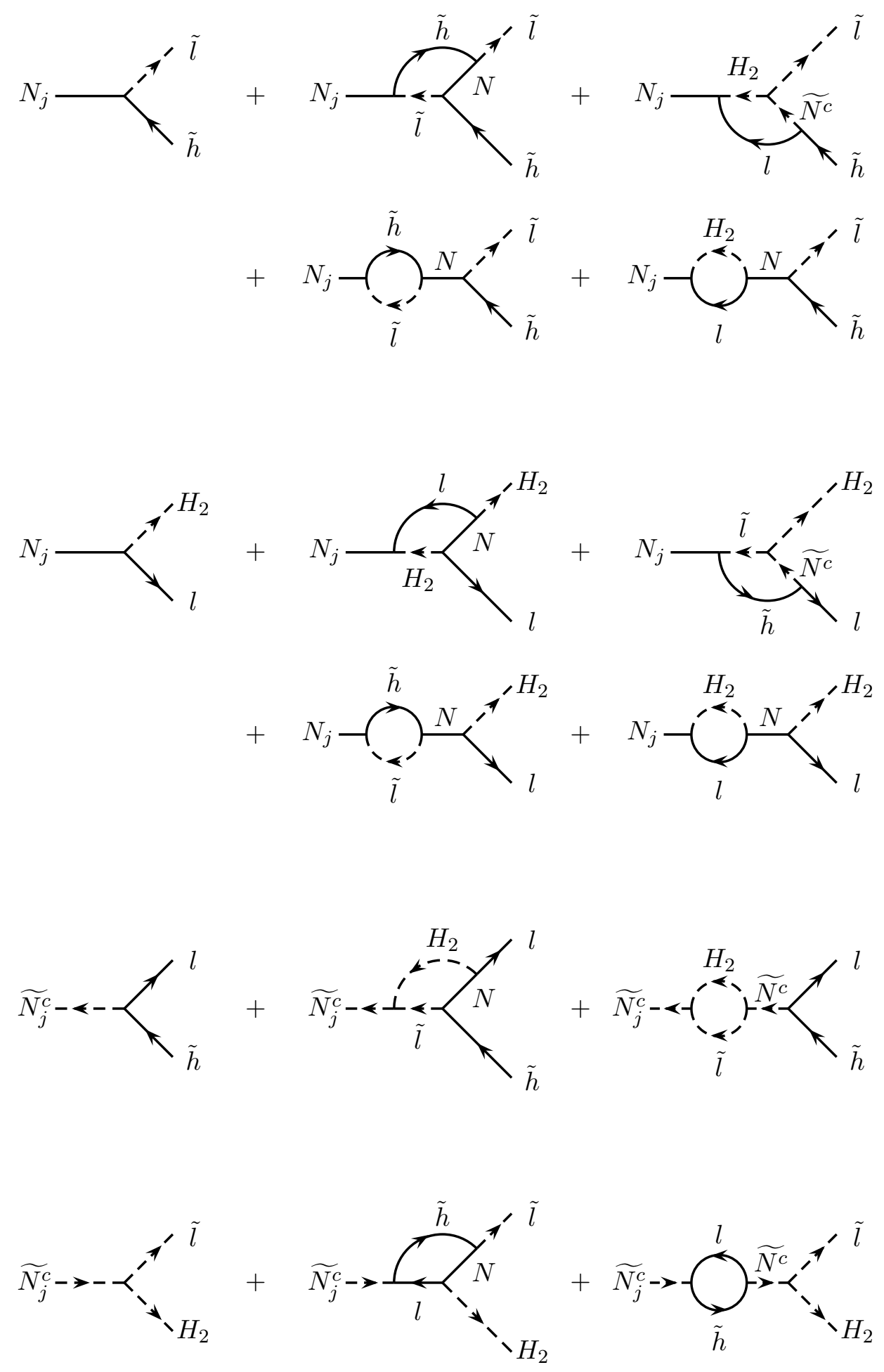

Figure 3.2: Decay modes of the right-handed Majorana neutrinos and their scalar partners. 
All these decay modes are $C P$ violating, the dominant contribution to $C P$ violation coming about through interference between the tree level and the one-loop diagrams shown in fig. 3.2. These one-loop diagrams can be summarized by the one-loop superfield diagrams in figs. 3.1]b and 3.1 c. Note that the non-renormalization theorems do not apply to these diagrams, since they are contributions to the D-term $N_{j}^{c} \bar{L}_{i} \epsilon \bar{H}_{2}$. Using the superspace Feynman rules discussed in section 2.6 the diagram in fig. 3.1b yields the following contribution to the $T$-matrix

$$
\begin{aligned}
i T_{N}^{(b)} & =\sum_{n}\left(\lambda_{\nu}^{\dagger} \lambda_{\nu}\right)_{n j}\left(\lambda_{\nu}^{\dagger}\right)_{n i} \int d^{8} z_{1} d^{8} z_{2} d^{8} z_{3} N_{j}^{c}\left(z_{1}\right) \bar{L}_{i}\left(z_{2}\right) \epsilon \bar{H}_{2}\left(z_{3}\right) \times \\
& \times\left[\frac{\bar{D}^{2}\left(z_{1}\right)}{4} \frac{\delta^{8}\left(z_{1}-z_{2}\right)}{\square_{1}-i \varepsilon}\right]\left[\frac{D^{2}\left(z_{3}\right)}{4} \frac{\delta^{8}\left(z_{1}-z_{3}\right)}{\square_{1}-i \varepsilon}\right]\left[-\frac{M_{n}}{16} \frac{D^{2}\left(z_{2}\right) \bar{D}^{2}\left(z_{2}\right)}{\square_{2}} \frac{\delta^{8}\left(z_{2}-z_{3}\right)}{\square_{2}+M_{n}^{2}-i \varepsilon}\right] .
\end{aligned}
$$

After partial integration of the $D_{\alpha}\left(z_{3}\right)$ derivatives, the only non-vanishing contribution reads

$$
\begin{aligned}
i T_{N}^{(b)}= & \sum_{n}\left(\lambda_{\nu}^{\dagger} \lambda_{\nu}\right)_{n j}\left(\lambda_{\nu}^{\dagger}\right)_{n i} \int d^{8} z_{1} d^{8} z_{2} d^{8} z_{3} N_{j}^{c}\left(z_{1}\right) \bar{L}_{i}\left(z_{2}\right) \epsilon \bar{H}_{2}\left(z_{3}\right) \frac{1}{4} \frac{\delta\left(z_{1}-z_{3}\right)}{\square_{1}-i \varepsilon} \times \\
& \times\left[\frac{\bar{D}^{2}\left(z_{1}\right)}{4} \frac{\delta\left(z_{1}-z_{2}\right)}{\square_{1}-i \varepsilon}\right]\left[-\frac{M_{n}}{16} \frac{D^{2}\left(z_{2}\right) \bar{D}^{2}\left(z_{2}\right)}{\square_{2}} D^{2}\left(z_{3}\right) \frac{\delta\left(z_{2}-z_{3}\right)}{\square_{2}+M_{n}^{2}-i \varepsilon}\right] \\
= & \left.\sum_{n}\left(\lambda_{\nu}^{\dagger} \lambda_{\nu}\right)_{n j}\left(\lambda_{\nu}^{\dagger}\right)_{n i}\right] d^{8} z_{1} d^{8} z_{2} d^{8} z_{3} N_{j}^{c}\left(z_{1}\right) \bar{L}_{i}\left(z_{2}\right) \epsilon \bar{H}_{2}\left(z_{3}\right) \frac{1}{4} \frac{\delta\left(z_{1}-z_{3}\right)}{\square_{1}-i \varepsilon} \times \\
& \times\left[\frac{\bar{D}^{2}\left(z_{2}\right)}{4} \frac{\delta\left(z_{1}-z_{2}\right)}{\square_{1}-i \varepsilon}\right]\left[M_{n} D^{2}\left(z_{2}\right) \frac{\delta\left(z_{2}-z_{3}\right)}{\square_{2}+M_{n}^{2}-i \varepsilon}\right],
\end{aligned}
$$

where we have used eqs. (2.61), (2.62) and (2.23) in the last step. Now we can perform the $d^{2} \theta_{3} d^{2} \overline{\theta_{3}}$ integration,

$$
\begin{aligned}
i T_{N}^{(b)}= & \sum_{n}\left(\lambda_{\nu}^{\dagger} \lambda_{\nu}\right)_{n j}\left(\lambda_{\nu}^{\dagger}\right)_{n i} \int d^{8} z_{1} d^{8} z_{2} d^{4} x_{3} N_{j}^{c}\left(z_{1}\right) \bar{L}_{i}\left(z_{2}\right) \epsilon \bar{H}_{2}\left(z_{1}\right) \frac{1}{4} \frac{\delta^{4}\left(x_{1}-x_{3}\right)}{\square_{1}-i \varepsilon} \times \\
& \times\left[\frac{\bar{D}^{2}\left(z_{2}\right)}{4} \frac{\delta^{8}\left(z_{1}-z_{2}\right)}{\square_{1}-i \varepsilon}\right]\left[M_{n} D^{2}\left(z_{2}\right) \frac{\delta^{8}\left(z_{2}-z_{1}\right)}{\square_{2}+M_{n}^{2}-i \varepsilon}\right] .
\end{aligned}
$$

Similarly, we can partially integrate the $\bar{D}^{2}\left(z_{2}\right)$ derivatives, and perform the $d^{2} \theta_{2} d^{2} \overline{\theta_{2}}$ integrations after having used eq. (2.100) to remove all covariant derivatives. Then $i T^{(b)}$ reads

$$
\begin{aligned}
i T_{N}^{(b)}= & \sum_{n} M_{n}\left(\lambda_{\nu}^{\dagger} \lambda_{\nu}\right)_{n j}\left(\lambda_{\nu}^{\dagger}\right)_{n i} \int d^{4} x_{1} d^{4} x_{2} d^{4} x_{3} \frac{\delta^{4}\left(x_{1}-x_{3}\right)}{\square_{1}-i \varepsilon} \frac{\delta^{4}\left(x_{1}-x_{2}\right)}{\square_{1}-i \varepsilon} \frac{\delta^{4}\left(x_{2}-x_{3}\right)}{\square_{2}+M_{n}^{2}-i \varepsilon} \times \\
& \times \int d^{2} \theta d^{2} \bar{\theta} N_{j}^{c}\left(x_{1}, \theta, \bar{\theta}\right) \bar{L}_{i}\left(x_{2}, \theta, \bar{\theta}\right) \epsilon \bar{H}_{2}\left(x_{3}, \theta, \bar{\theta}\right) .
\end{aligned}
$$


By Fourier transforming the loop propagators, we get

$$
\begin{aligned}
i T_{N}^{(b)}= & -\sum_{n} M_{n}\left(\lambda_{\nu}^{\dagger} \lambda_{\nu}\right)_{n j}\left(\lambda_{\nu}^{\dagger}\right)_{n i} \times \\
& \times \int d^{4} x_{1} d^{4} x_{2} d^{4} x_{3} \int \frac{d^{4} k_{1}}{(2 \pi)^{4}} \frac{d^{4} k_{2}}{(2 \pi)^{4}} \frac{d^{4} k_{3}}{(2 \pi)^{4}} \frac{\mathrm{e}^{i x_{1}\left(k_{1}+k_{2}\right)} \mathrm{e}^{i x_{2}\left(k_{3}-k_{2}\right)} \mathrm{e}^{-i x_{3}\left(k_{1}+k_{3}\right)}}{\left(k_{1}^{2}+i \varepsilon\right)\left(k_{2}^{2}+i \varepsilon\right)\left(k_{3}^{2}-M_{n}^{2}+i \varepsilon\right)} \\
& \times \int d^{2} \theta d^{2} \bar{\theta} N_{j}^{c}\left(x_{1}, \theta, \bar{\theta}\right) \bar{L}_{i}\left(x_{2}, \theta, \bar{\theta}\right) \epsilon \bar{H}_{2}\left(x_{3}, \theta, \bar{\theta}\right) .
\end{aligned}
$$

Analogously, we can compute the contribution of the diagram in fig. 3.1c to the $T$-matrix,

$$
\begin{aligned}
i T_{N}^{(c)}= & 2 \sum_{n} M_{n}\left(\lambda_{\nu}^{\dagger} \lambda_{\nu}\right)_{n j}\left(\lambda_{\nu}^{\dagger}\right)_{n i} \int d^{4} x_{1} d^{4} x_{2} d^{4} x_{3}\left(\frac{\delta^{4}\left(x_{1}-x_{2}\right)}{\square_{1}-i \varepsilon}\right)^{2} \frac{\delta^{4}\left(x_{2}-x_{3}\right)}{\square_{3}+M_{n}^{2}-i \varepsilon} \times \\
& \times \int d^{2} \theta d^{2} \bar{\theta} N_{j}^{c}\left(x_{1}, \theta, \bar{\theta}\right) \bar{L}_{i}\left(x_{3}, \theta, \bar{\theta}\right) \epsilon \bar{H}_{2}\left(x_{3}, \theta, \bar{\theta}\right) .
\end{aligned}
$$

Again using the Fourier representation for the propagators, we find

$$
\begin{aligned}
i T_{N}^{(c)}= & \frac{-i}{8 \pi^{2}} \sum_{n} M_{n}\left(\lambda_{\nu}^{\dagger} \lambda_{\nu}\right)_{n j}\left(\lambda_{\nu}^{\dagger}\right)_{n i} \int \frac{d^{4} k}{(2 \pi)^{4}} \frac{B_{0}(-k, 0,0)}{k^{2}-M_{n}^{2}+i \varepsilon} \int d^{4} x_{1} d^{4} x_{3} \mathrm{e}^{i k\left(x_{1}-x_{3}\right)} \times \\
& \times \int d^{2} \theta d^{2} \bar{\theta} N_{j}^{c}\left(x_{1}, \theta, \bar{\theta}\right) \bar{L}_{i}\left(x_{3}, \theta, \bar{\theta}\right) \epsilon \bar{H}_{2}\left(x_{3}, \theta, \bar{\theta}\right)
\end{aligned}
$$

where $B_{0}(-k, 0,0)$ is the massless two-point scalar integral defined in eq. (A.5). One-loop corrections to the decay amplitudes are given by the matrix elements of $i T_{N}^{(b)}+i T_{N}^{(c)}$.

Interference between these one-loop diagrams and the tree-level amplitudes gives rise to $C P$ asymmetries in the different decay channels of $N_{j}$ and $\widetilde{N_{j}^{c}}$, which can all be expressed by the same $C P$ violation parameter $\varepsilon_{j}$,

$$
\begin{aligned}
\varepsilon_{j}:= & \frac{\Gamma\left(N_{j} \rightarrow \widetilde{l}+\overline{\widetilde{h}}\right)-\Gamma\left(N_{j} \rightarrow \widetilde{l}^{\dagger}+\widetilde{h}\right)}{\Gamma\left(N_{j} \rightarrow \widetilde{l}+\overline{\widetilde{h}}\right)+\Gamma\left(N_{j} \rightarrow \widetilde{l}^{\dagger}+\widetilde{h}\right)}=\frac{\Gamma\left(N_{j} \rightarrow l+H_{2}\right)-\Gamma\left(N_{j} \rightarrow \bar{l}+H_{2}^{\dagger}\right)}{\Gamma\left(N_{j} \rightarrow l+H_{2}\right)+\Gamma\left(N_{j} \rightarrow \bar{l}+H_{2}^{\dagger}\right)} \\
= & \frac{\Gamma\left(\widetilde{N_{j}^{c}} \rightarrow l+\widetilde{\widetilde{h}}\right)-\Gamma\left(\widetilde{N_{j}^{c}} \rightarrow \bar{l}+\widetilde{h}\right)}{\Gamma\left(\widetilde{N}_{j}^{c} \rightarrow l+\overline{\widetilde{h}}\right)+\Gamma\left(\widetilde{N_{j}^{c}} \rightarrow \bar{l}+\widetilde{h}\right)}=\frac{\Gamma\left(\widetilde{N_{j}^{c}} \rightarrow \widetilde{l}+H_{2}\right)-\Gamma\left({\widetilde{N_{j}^{c}}}^{\dagger} \rightarrow \widetilde{l}^{\dagger}+H_{2}^{\dagger}\right)}{\Gamma\left(\widetilde{N_{j}^{c}} \rightarrow \widetilde{l}+H_{2}\right)+\Gamma\left({\widetilde{N_{j}^{c}}}^{\dagger} \rightarrow \widetilde{l}^{\dagger}+H_{2}^{\dagger}\right)} \\
= & -\frac{1}{8 \pi v_{2}^{2}} \frac{1}{\left(m_{D}^{\dagger} m_{D}\right)_{j j}} \sum_{n \neq j} \operatorname{Im}\left[\left(m_{D}^{\dagger} m_{D}\right)_{n j}^{2}\right] g\left(\frac{a_{n}}{a_{j}}\right), \\
& \quad \text { with } g(x)=\sqrt{x}\left[\ln \left(\frac{1+x}{x}\right)+\frac{2}{x-1}\right] \approx \frac{3}{\sqrt{x}} \text { for } x \gg 1 .
\end{aligned}
$$

Here $n$ is the flavour index of the heavy (s)neutrino in the loop. This result agrees with the one in ref. [18 and is of the same order as the $C P$ asymmetry in ref. [16]. 

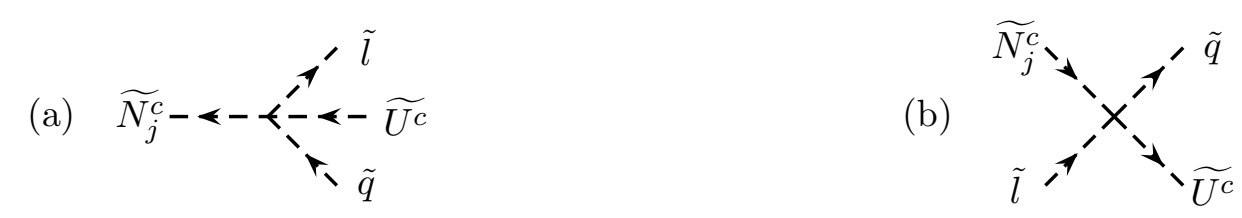

Figure 3.3: Contributions of the scalar potential to the decay width and the interactions of a scalar neutrino.

With $\varepsilon_{j}$ we can parametrize the reaction densities for the decays and inverse decays in the following way

$$
\begin{aligned}
\frac{1}{4}\left(1+\varepsilon_{j}\right) \gamma_{N_{j}} & =\gamma\left(N_{j} \rightarrow \widetilde{l}+\overline{\widetilde{h}}\right)=\gamma\left(N_{j} \rightarrow l+H_{2}\right) \\
& =\gamma\left(\widetilde{l}^{\dagger}+\widetilde{h} \rightarrow N_{j}\right)=\gamma\left(\bar{l}+H_{2}^{\dagger} \rightarrow N_{j}\right) \\
\frac{1}{4}\left(1-\varepsilon_{j}\right) \gamma_{N_{j}} & =\gamma\left(N_{j} \rightarrow \widetilde{l}^{\dagger}+\widetilde{h}\right)=\gamma\left(N_{j} \rightarrow \bar{l}+H_{2}^{\dagger}\right) \\
& =\gamma\left(\widetilde{l}+\overline{\widetilde{h}} \rightarrow N_{j}\right)=\gamma\left(l+H_{2} \rightarrow N_{j}\right) \\
\frac{1}{2}\left(1+\varepsilon_{j}\right) \gamma_{\bar{N}_{j}^{c}}^{(2)} & =\gamma\left(\widetilde{N_{j}^{c}} \rightarrow \widetilde{l}+H_{2}\right)=\gamma\left(\widetilde{N_{j}^{c}} \rightarrow l+\widetilde{\widetilde{h}}\right) \\
& =\gamma\left(\widetilde{l}^{\dagger}+H_{2}^{\dagger} \rightarrow \widetilde{N}_{j}^{\dagger}\right)=\gamma\left(\bar{l}+\widetilde{h} \rightarrow \widetilde{N_{j}^{c}}\right) \\
\frac{1}{2}\left(1-\varepsilon_{j}\right) \gamma_{\widetilde{N}_{j}^{c}}^{(2)} & =\gamma\left(\widetilde{N_{j}^{c}} \rightarrow \widetilde{l}^{\dagger}+H_{2}^{\dagger}\right)=\gamma\left(\widetilde{N_{j}^{c}} \rightarrow \bar{l}+\widetilde{h}\right) \\
& =\gamma\left(\widetilde{l}+H_{2} \rightarrow \widetilde{N_{j}^{c}}\right)=\gamma\left(l+\widetilde{\widetilde{h}} \rightarrow \widetilde{N_{j}^{c}}\right) .
\end{aligned}
$$

Additionally, the scalar potential contains quartic scalar couplings, which enable the decay of $\widetilde{N_{j}^{c}}$ into three particles via the diagram shown in fig. 3.3a. This is just the contribution of the auxiliary field $F_{H_{2}}$ given in eq. (3.16) to the superfield decay amplitude in fig. 3.1a. The partial width for this decay is given by

$$
\Gamma_{\widetilde{N}_{j}^{c}}^{(3)}:=\Gamma\left({\widetilde{N_{j}^{c}}}^{\dagger} \rightarrow \widetilde{l}+{\widetilde{U^{c}}}^{\dagger}+\widetilde{q}^{\dagger}\right)=\frac{3 \alpha_{u} M_{j}}{64 \pi^{2}} \frac{\left(m_{D}^{\dagger} m_{D}\right)_{j j}}{v_{2}^{2}} \quad \text { with } \quad \alpha_{u}=\frac{\operatorname{Tr}\left(\lambda_{u}^{\dagger} \lambda_{u}\right)}{4 \pi},
$$

and the corresponding reaction density reads

$$
\gamma_{\overline{N_{j}^{c}}}^{(3)}=\frac{3 \alpha_{u} M_{1}^{4}}{128 \pi^{4}} \frac{\left(m_{D}^{\dagger} m_{D}\right)_{j j}}{v_{2}^{2}} \frac{a_{j} \sqrt{a_{j}}}{z} \mathrm{~K}_{1}\left(z \sqrt{a_{j}}\right)=\frac{3 \alpha_{u}}{16 \pi} \gamma_{\overline{N_{j}^{c}}}^{(2)} .
$$

Since the Yukawa coupling of the top quark and its scalar partner is large, $\alpha_{u}$ can be of order one. But even then $\gamma_{N_{j}^{c}}^{(3)}$ is much smaller than $\gamma_{\frac{(2)}{N_{j}^{c}}}$. Hence, the three particle decays give only a small 


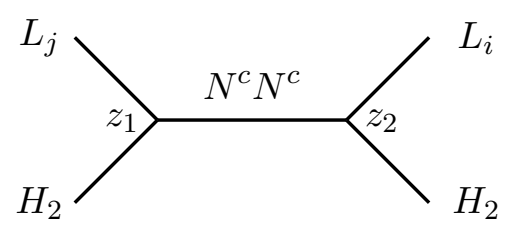

Figure 3.4: Lepton number violating scatterings mediated by a neutrino superfield.

correction, which we have taken into account for completeness. However, we have neglected the $C P$ asymmetry in this decay which comes about through the one-loop diagrams in fig. 3.1.

Furthermore, we have neglected the leptonic auxiliary field $F_{L_{i}}$ given in eq. (3.18), since its contribution to the sneutrino decay width will be of order $\left(\lambda_{\nu}^{\dagger} \lambda_{l}^{2} \lambda_{\nu}\right)_{j j}$, i.e. much smaller than the other partial decay widths, at least for the lightest sneutrino $(j=1)$.

The dimensionless squared total decay widths of $N_{j}$ and $\widetilde{N_{j}^{c}}$ are then finally given by

$$
\begin{aligned}
c_{j} & :=\left(\frac{\Gamma_{N_{j}}}{M_{1}}\right)^{2}=\frac{a_{j}}{16 \pi^{2}} \frac{\left(m_{D}^{\dagger} m_{D}\right)_{j j}^{2}}{v_{2}^{4}}, \\
\widetilde{c_{j}} & :=\left(\frac{\Gamma_{N_{j}^{c}}^{(2)}+\Gamma_{\widetilde{N_{j}^{c}}}^{(3)}}{M_{1}}\right)^{2}=\frac{a_{j}}{16 \pi^{2}} \frac{\left(m_{D}^{\dagger} m_{D}\right)_{j j}^{2}}{v_{2}^{4}}\left[1+\frac{3 \alpha_{u}}{16 \pi}\right]^{2} .
\end{aligned}
$$

The vertex in fig. 3.3a also gives $2 \rightarrow 2$ scattering processes involving one scalar neutrino, like $\widetilde{N_{j}^{c}}+\widetilde{l} \rightarrow \widetilde{q}+\widetilde{U^{c}}$ (cf. fig. 3.3b). The reduced cross section for this process reads

$$
\hat{\sigma}_{22_{j}}(x)=3 \alpha_{u} \frac{\left(m_{D}^{\dagger} m_{D}\right)_{j j}}{v_{2}^{2}} \frac{x-a_{j}}{x} .
$$

For the processes $\widetilde{N_{j}^{c}}+\widetilde{q}^{\dagger} \rightarrow \widetilde{l}^{\dagger}+\widetilde{U^{c}}$ and $\widetilde{N_{j}^{c}}+\widetilde{U^{c}} \widetilde{l}^{\dagger}+\widetilde{q}$, the corresponding back reactions and the $C P$ conjugated processes we find the same result. The corresponding reaction density can then be calculated according to eq. (D.16). One finds

$$
\gamma_{22_{j}}(z)=\frac{3 \alpha_{u} M_{1}^{4}}{16 \pi^{4}} \frac{\left(m_{D}^{\dagger} m_{D}\right)_{j j}}{v_{2}^{2}} \frac{\sqrt{a_{j}}}{z^{3}} \mathrm{~K}_{1}\left(z \sqrt{a_{j}}\right)=\frac{3 \alpha_{u}}{4 \pi a_{j} z^{2}} \gamma_{N_{j}}(z)
$$

Hence, $\gamma_{22_{j}}$ will be much larger than $\gamma_{N_{j}}$ and $\gamma_{\widetilde{N}_{j}^{c}}^{(2)}$ for small $a_{j} z^{2}$, i.e. for high temperatures $T \gg M_{j}$. Together with similar scatterings, which we are going to discuss in section 3.4, these processes will therefore be very effective in bringing the heavy (s)neutrinos into thermal equilibrium at high temperatures where decays and inverse decays are suppressed by a time dilatation factor. 


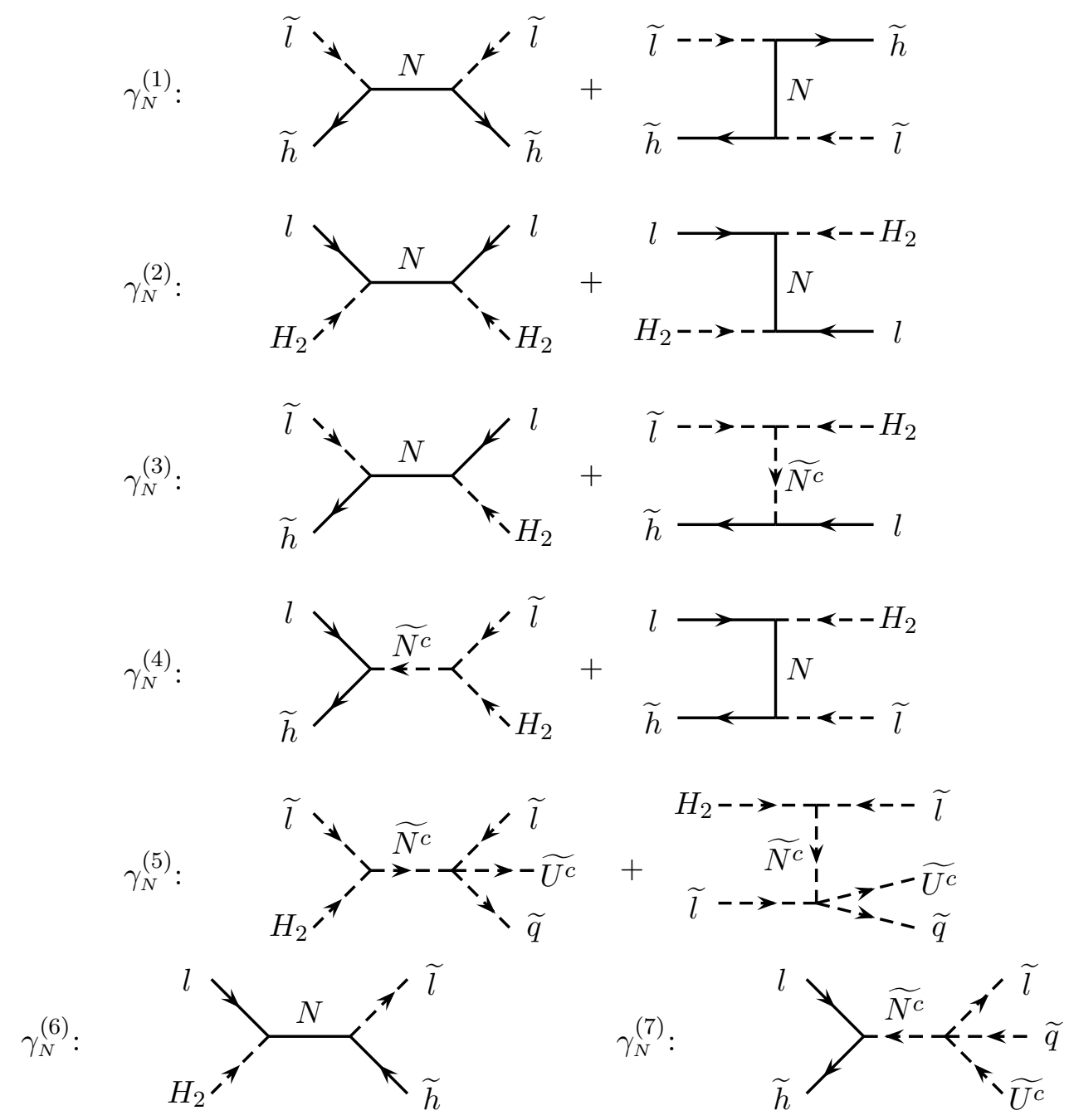

Figure 3.5: L violating processes mediated by a virtual Majorana neutrino or its scalar partner.

\subsection{Lepton Number Violating Scatterings Mediated by Right- Handed Neutrinos}

Using the tree level vertices from figs. 3.2 and 3.3 as building blocks we can construct lepton number violating scatterings mediated by a heavy (s)neutrino. Although of higher order than the tree level decays, these diagrams have to be taken into consideration to avoid the generation of an asymmetry in thermal equilibrium, which is forbidden by $C P T$ invariance 35,36 .

All these processes can be summarized in the configuration space superfield diagram shown in 


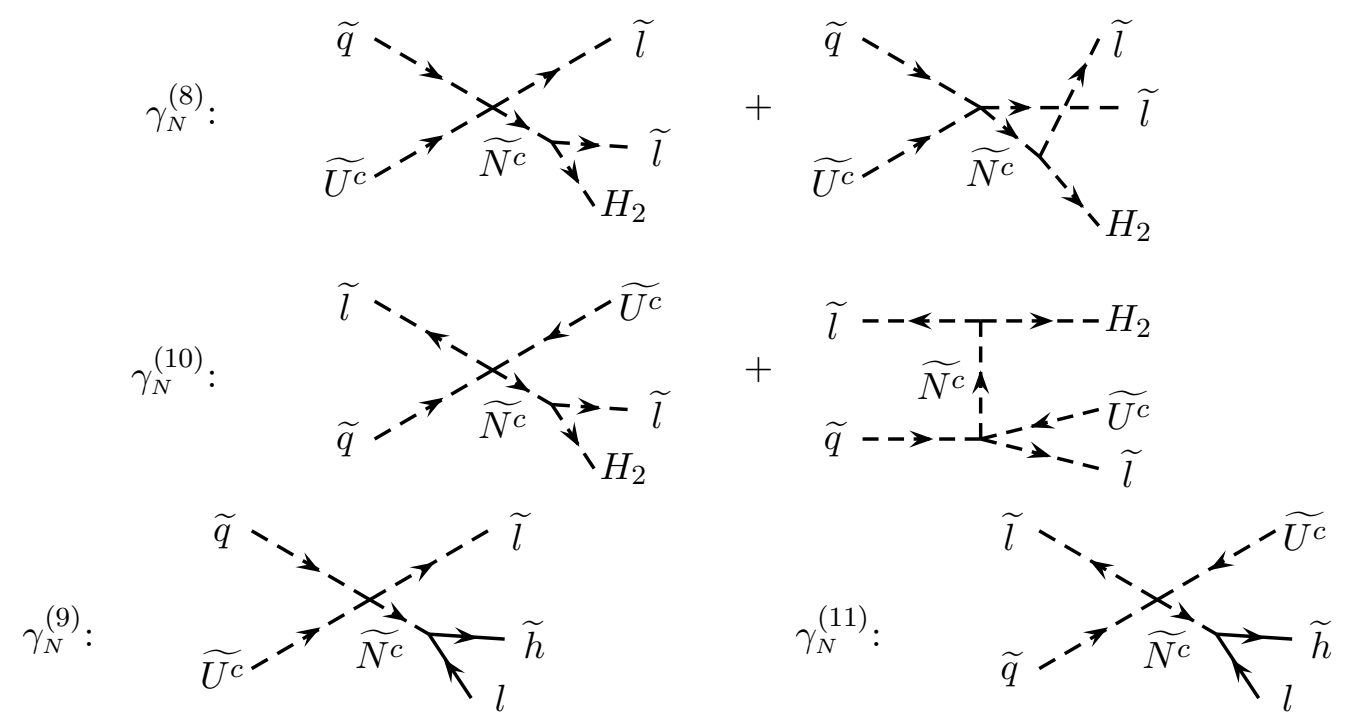

Figure 3.6: Diagrams contributing to the lepton number violating scatterings via heavy sneutrino exchange.

fig. 3.4. It yields the following contribution to the $T$-matrix

$$
\begin{aligned}
i T_{\Delta L}= & \frac{i}{2} \sum_{k}\left(\lambda_{\nu}\right)_{i k}\left(\lambda_{\nu}\right)_{j k} \int d^{4} x_{1} d^{4} x_{2} \int \frac{d^{4} k}{(2 \pi)^{4}} \frac{\mathrm{e}^{i k\left(x_{1}-x_{2}\right)}}{k^{2}-M_{k}^{2}+i \varepsilon} \times \\
& \times \int d^{2} \theta d^{2} \bar{\theta} L_{j}\left(x_{1}, \theta, \bar{\theta}\right) \epsilon H_{2}\left(x_{1}, \theta, \bar{\theta}\right) \frac{M_{k}}{4} \frac{D_{2}^{2}}{\square_{2}} L_{i}\left(x_{2}, \theta, \bar{\theta}\right) \epsilon H_{2}\left(x_{2}, \theta, \bar{\theta}\right) .
\end{aligned}
$$

The superfield product can be evaluated with eq. (B.56). The matrix elements of $i T_{\Delta L}$ then correspond to the component field processes that we are going to discuss in the following. In this section we will only mention the different processes which have to be considered. The corresponding reduced cross sections can be found in appendix $\mathrm{E}$ and the reaction densities are discussed in appendix $\mathrm{F}$.

By combining two of the decay vertices (cf. fig. 3.2 and fig. 3.3a) one gets the processes that we have shown in fig. 3.5 and the corresponding $C P$ conjugated processes. We will use the following abbreviations for the reaction densities

$$
\begin{array}{rlrl}
\gamma_{N}^{(1)} & =\gamma\left(\widetilde{l}+\overline{\widetilde{h}} \leftrightarrow \widetilde{l}^{\dagger}+\widetilde{h}\right), & \gamma_{N}^{(2)}=\gamma\left(l+H_{2} \leftrightarrow \bar{l}+H_{2}^{\dagger}\right), \\
\gamma_{N}^{(3)}=\gamma\left(\widetilde{l}+\overline{\widetilde{h}} \leftrightarrow \bar{l}+H_{2}^{\dagger}\right), & \gamma_{N}^{(4)}=\gamma\left(l+\overline{\widetilde{h}} \leftrightarrow \widetilde{l}^{\dagger}+H_{2}^{\dagger}\right), \\
\gamma_{N}^{(5)}=\gamma\left(\widetilde{l}+H_{2} \leftrightarrow \widetilde{l}^{\dagger}+\widetilde{U^{c}}+\widetilde{q}\right), & \gamma_{N}^{(6)}=\gamma\left(l+H_{2} \leftrightarrow \widetilde{l}+\overline{\widetilde{h}}\right), \\
\gamma_{N}^{(7)}=\gamma\left(l+\widetilde{\widetilde{h}} \leftrightarrow \widetilde{l}+\widetilde{q}^{\dagger}+\widetilde{U}^{\dagger}\right) . &
\end{array}
$$




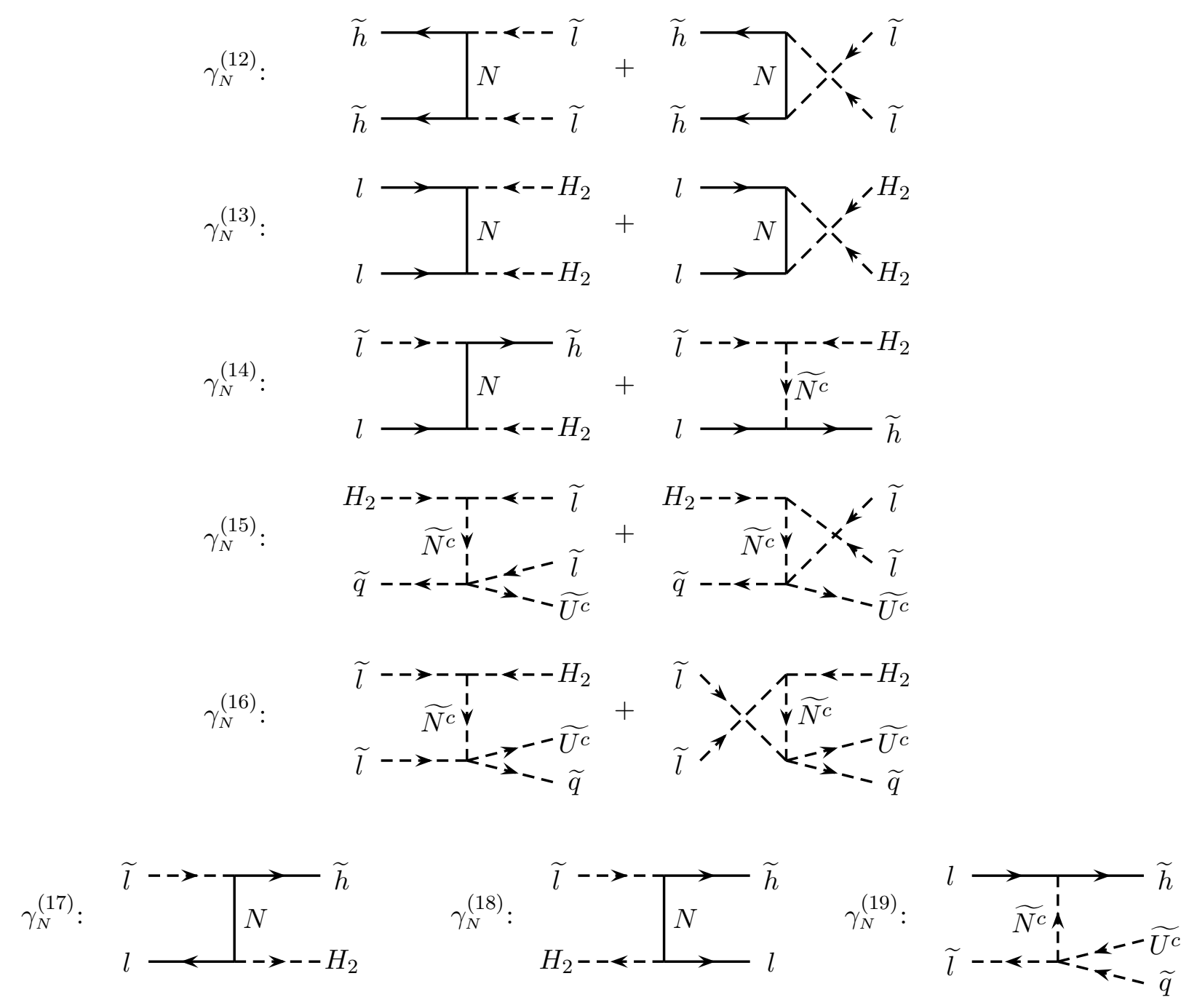

Figure 3.7: L violating processes mediated by a right-handed (s)neutrino in the t-channel.

The contributions from on-shell (s)neutrinos contained in these reactions have already been taken into account as inverse decay followed by a decay. Hence, one has to subtract the contributions from real intermediate states to avoid a double counting of reactions [36].

From the scattering vertex in fig. 3.3 b and the decay vertices we can construct the following processes

$$
\begin{aligned}
\gamma_{N}^{(8)} & =\gamma\left(\widetilde{U^{c}}+\widetilde{q} \leftrightarrow \widetilde{l}+\widetilde{l}+H_{2}\right), \quad \gamma_{N}^{(9)}=\gamma\left(\widetilde{q}+\widetilde{U^{c}} \leftrightarrow \widetilde{l}+\bar{l}+\widetilde{h}\right), \\
\gamma_{N}^{(10)} & =\gamma\left(\widetilde{l}^{\dagger}+\widetilde{q} \leftrightarrow \widetilde{l}+{\widetilde{U^{c}}}^{\dagger}+H_{2}\right)=\gamma\left(\widetilde{l}^{\dagger}+\widetilde{U^{c}} \leftrightarrow \widetilde{l}+\widetilde{q}^{\dagger}+H_{2}\right), \\
\gamma_{N}^{(11)} & =\gamma\left(\widetilde{l}^{\dagger}+\widetilde{q} \leftrightarrow \bar{l}+\widetilde{h}+{\widetilde{U^{c}}}^{\dagger}\right)=\gamma\left(\widetilde{l}^{\dagger}+\widetilde{U^{c}} \leftrightarrow \bar{l}+\widetilde{h}+\widetilde{q}^{\dagger}\right) .
\end{aligned}
$$


In fig. 3.6 we have shown one typical diagram for each of these reaction densities. Again, these diagrams have on-shell contributions which have to be subtracted, since they can be described as decay of a sneutrino which has been produced in a scattering process.

Up to now we have only considered processes with a neutrino or its scalar partner in the $s$ channel. In fig. 3.7 we have shown a selection of diagrams without on-shell contributions. The corresponding reaction densities will be denoted by

$$
\begin{aligned}
& \gamma_{N}^{(12)}=\gamma\left(\overline{\widetilde{h}}+\overline{\widetilde{h}} \leftrightarrow \widetilde{l}^{\dagger}+\widetilde{l}^{\dagger}\right), \quad \quad \gamma_{N}^{(13)}=\gamma\left(l+l \leftrightarrow H_{2}^{\dagger}+H_{2}^{\dagger}\right), \\
& \gamma_{N}^{(14)}=\gamma\left(\widetilde{l}+l \leftrightarrow \widetilde{h}+H_{2}^{\dagger}\right), \quad \gamma_{N}^{(16)}=\gamma\left(\widetilde{l}+\widetilde{l} \leftrightarrow \widetilde{U^{c}}+\widetilde{q}+H_{2}^{\dagger}\right), \\
& \gamma_{N}^{(15)}=\gamma\left(H_{2}+\widetilde{q}^{\dagger} \leftrightarrow \widetilde{l}^{\dagger}+\widetilde{l}^{\dagger}+\widetilde{U^{c}}\right)=\gamma\left(H_{2}+{\widetilde{U^{c}}}^{\dagger} \leftrightarrow \widetilde{l}^{\dagger}+\widetilde{l}^{\dagger}+\widetilde{q}\right) \\
& \gamma_{N}^{(17)}=\gamma\left(\widetilde{l}+\bar{l} \leftrightarrow \widetilde{h}+H_{2}\right), \quad \quad \gamma_{N}^{(18)}=\gamma\left(\widetilde{l}+H_{2}^{\dagger} \leftrightarrow \widetilde{h}+l\right) \\
& \gamma_{N}^{(19)}=\gamma\left(l+\widetilde{l}^{\dagger} \leftrightarrow \widetilde{h}+\widetilde{q}^{\dagger}+{\widetilde{U^{c}}}^{\dagger}\right)=\gamma\left(l+\widetilde{q} \leftrightarrow \widetilde{l}+{\widetilde{U^{c}}}^{\dagger}+\widetilde{h}\right) \\
& =\gamma\left(l+\widetilde{U^{c}} \leftrightarrow \widetilde{l}+\widetilde{q}^{\dagger}+\widetilde{h}\right)=\gamma\left(\widetilde{l}^{\dagger}+\overline{\widetilde{h}} \leftrightarrow \bar{l}+\widetilde{q}^{\dagger}+{\widetilde{U^{c}}}^{\dagger}\right) \\
& =\gamma\left(\widetilde{q}+\overline{\widetilde{h}} \leftrightarrow \bar{l}+\widetilde{l}+{\widetilde{U^{c}}}^{\dagger}\right)=\gamma\left(\widetilde{U^{c}}+\overline{\widetilde{h}} \leftrightarrow \bar{l}+\widetilde{l}+\widetilde{q}^{\dagger}\right) .
\end{aligned}
$$

At first sight one may think that these diagrams could be neglected, since they are suppressed at intermediate temperatures, i.e. intermediate energies $x \approx a_{j}$. However, they give an important contribution to the effective lepton number violating interactions at low energies and therefore have to be taken into consideration.

\subsection{Interactions with a Top or a Stop}

The Yukawa coupling of the top quark is large. Thus we have to take into account lepton number violating interactions of a right-handed neutrino with a top quark or its scalar partner. In addition

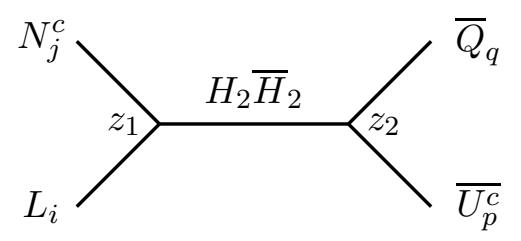

Figure 3.8: Neutrino-(s)top scattering in configuration superspace. $i, j, q$ and $p$ are flavour indices. 


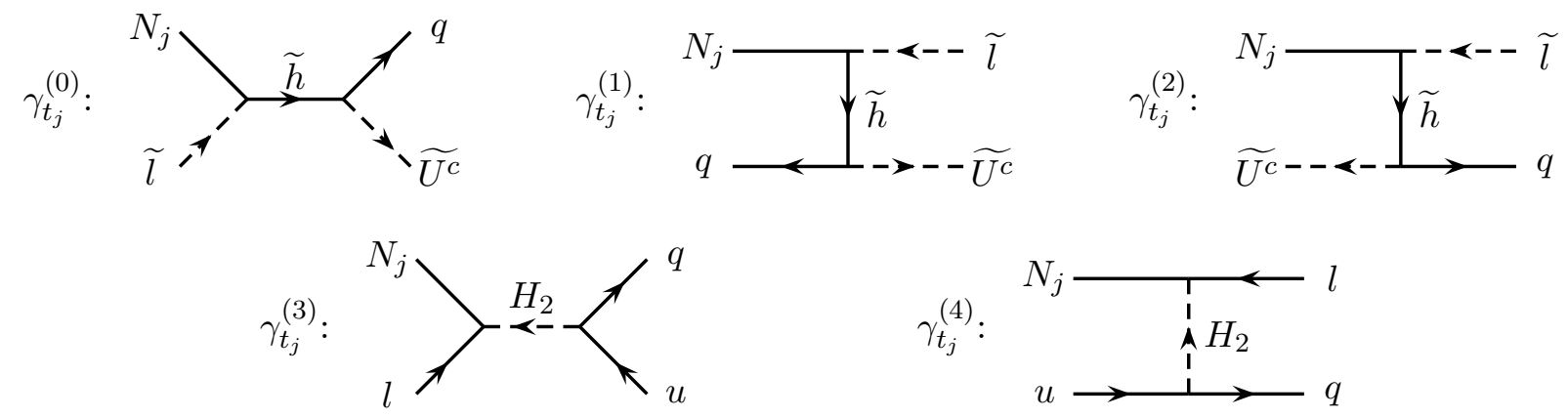

Figure 3.9: Neutrino-(s)top scattering.

to the processes already considered in section 3.2 (cf. fig. 3.3 b), we have the superfield diagram shown in fig.3.8, which gives the following contribution to the $T$-matrix

$$
\begin{aligned}
i T_{t_{j}}= & -i\left(\lambda_{\nu}\right)_{i j}\left(\lambda_{u}^{\dagger}\right)_{p q} \int d^{4} x_{1} d^{4} x_{2} \int \frac{d^{4} k}{(2 \pi)^{4}} \frac{\mathrm{e}^{i k\left(x_{1}-x_{2}\right)}}{k^{2}+i \varepsilon} \times \\
& \times \int d^{2} \theta d^{2} \bar{\theta} N_{j}^{c}\left(x_{1}, \theta, \bar{\theta}\right)\left(L_{i}\left(x_{1}, \theta, \bar{\theta}\right) \bar{Q}_{q}\left(x_{2}, \theta, \bar{\theta}\right)\right) \overline{U_{p}^{c}}\left(x_{2}, \theta, \bar{\theta}\right) .
\end{aligned}
$$

In component fields, we have the following processes with a Majorana neutrino $N_{j}$ as external line (cf. fig. 3.9)

$$
\begin{aligned}
& \gamma_{t_{j}}^{(0)}=\gamma\left(N_{j}+\widetilde{l} \leftrightarrow q+\widetilde{U^{c}}\right)=\gamma\left(N_{j}+\tilde{l} \leftrightarrow \widetilde{q}+\bar{u}\right), \\
& \gamma_{t_{j}}^{(1)}=\gamma\left(N_{j}+\bar{q} \leftrightarrow \widetilde{l}^{\dagger}+\widetilde{U^{c}}\right)=\gamma\left(N_{j}+u \leftrightarrow \widetilde{l}^{\dagger}+\widetilde{q}\right), \\
& \gamma_{t_{j}}^{(2)}=\gamma\left(N_{j}+{\widetilde{U^{c}}}^{\dagger} \leftrightarrow \widetilde{l}^{\dagger}+q\right)=\gamma\left(N_{j}+\widetilde{q}^{\dagger} \leftrightarrow \widetilde{l}^{\dagger}+\bar{u}\right), \\
& \gamma_{t_{j}}^{(3)}=\gamma\left(N_{j}+l \leftrightarrow q+\bar{u}\right), \\
& \gamma_{t_{j}}^{(4)}=\gamma\left(N_{j}+u \leftrightarrow \bar{l}+q\right)=\gamma\left(N_{j}+\bar{q} \leftrightarrow \bar{l}+\bar{u}\right) .
\end{aligned}
$$

At this order of perturbation theory these processes are $C P$ invariant. Hence, we have the same reaction densities for the $C P$ conjugated processes.

For the scalar neutrinos we have similarly (cf. fig. 3.10)

$$
\begin{aligned}
& \gamma_{t_{j}}^{(5)}=\gamma\left(\widetilde{N_{j}^{c}}+l \leftrightarrow q+\widetilde{U^{c}}\right)=\gamma\left(\widetilde{N_{j}^{c}}+l \leftrightarrow \widetilde{q}+\bar{u}\right), \\
& \left.\gamma_{t_{j}}^{(6)}=\gamma\left(\widetilde{N_{j}^{c}}+\widetilde{U^{c}}\right)^{\dagger} \leftrightarrow \bar{l}+q\right)=\gamma\left(\widetilde{N_{j}^{c}}+\widetilde{q}^{\dagger} \leftrightarrow \bar{l}+\bar{u}\right), \\
& \gamma_{t_{j}}^{(7)}=\gamma\left(\widetilde{N_{j}^{c}}+\bar{q} \leftrightarrow \bar{l}+\widetilde{U^{c}}\right)=\gamma\left(\widetilde{N_{j}^{c}}+u \leftrightarrow \bar{l}+\widetilde{q}\right),
\end{aligned}
$$




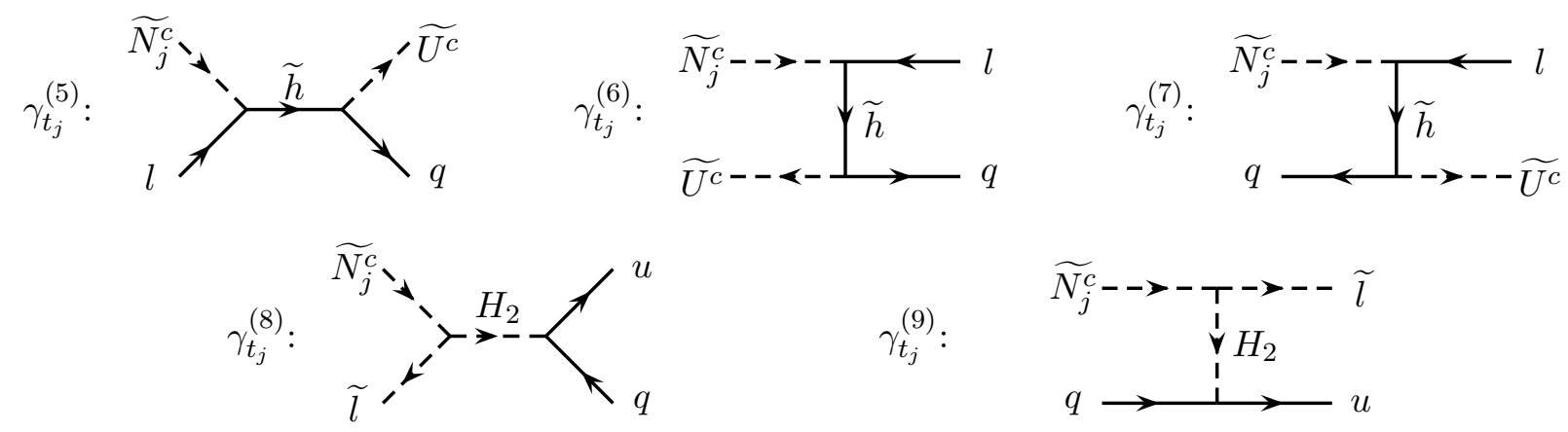

Figure 3.10: Sneutrino-(s)top scattering.

$$
\begin{aligned}
& \gamma_{t_{j}}^{(8)}=\gamma\left(\widetilde{N_{j}^{c}}+\widetilde{l}^{\dagger} \leftrightarrow \bar{q}+u\right), \\
& \gamma_{t_{j}}^{(9)}=\gamma\left(\widetilde{N_{j}^{c}}+q \leftrightarrow \widetilde{l}+u\right)=\gamma\left(\widetilde{N_{j}^{c}}+\bar{u} \leftrightarrow \widetilde{l}+\bar{q}\right) .
\end{aligned}
$$

The quartic scalar couplings from the scalar potential give additional $2 \rightarrow 3,3 \rightarrow 3$ and $2 \rightarrow 4$ processes, which can be neglected since they are phase space suppressed.

\subsection{Neutrino Pair Creation and Annihilation}

The Yukawa couplings of the right-handed neutrinos also allow lepton number conserving processes like neutrino pair creation and annihilation. The two superfield diagrams in fig. 3.11 yield the following contributions to the $T$-matrix

$$
\begin{aligned}
i T_{N N}^{(a)}= & -i\left(\lambda_{\nu}\right)_{k i}\left(\lambda_{\nu}^{\dagger}\right)_{j l} \int d^{4} x_{1} d^{4} x_{2} \int \frac{d^{4} k}{(2 \pi)^{4}} \frac{\mathrm{e}^{i k\left(x_{1}-x_{2}\right)}}{k^{2}+i \varepsilon} \times \\
& \times \int d^{2} \theta d^{2} \bar{\theta} N_{i}^{c}\left(x_{1}, \theta, \bar{\theta}\right)\left(L_{k}\left(x_{1}, \theta, \bar{\theta}\right) \bar{L}_{l}\left(x_{2}, \theta, \bar{\theta}\right)\right) \overline{N_{j}^{c}}\left(x_{2}, \theta, \bar{\theta}\right), \\
i T_{N N}^{(b)}= & i\left(\lambda_{\nu} \lambda_{\nu}^{\dagger}\right)_{j i} \int d^{4} x_{1} d^{4} x_{2} \int \frac{d^{4} k}{(2 \pi)^{4}} \frac{\mathrm{e}^{i k\left(x_{1}-x_{2}\right)}}{k^{2}+i \varepsilon} \times \\
& \times \int d^{2} \theta d^{2} \bar{\theta} N_{i}^{c}\left(x_{1}, \theta, \bar{\theta}\right)\left(H_{2}\left(x_{1}, \theta, \bar{\theta}\right) \bar{H}_{2}\left(x_{2}, \theta, \bar{\theta}\right)\right) \overline{N_{j}^{c}}\left(x_{2}, \theta, \bar{\theta}\right) .
\end{aligned}
$$

(a)

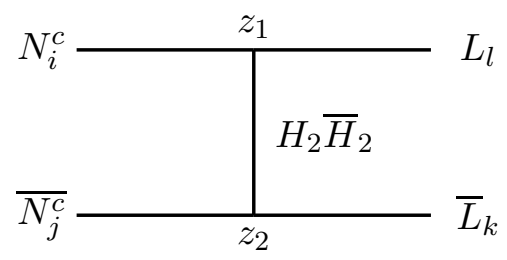

(b)

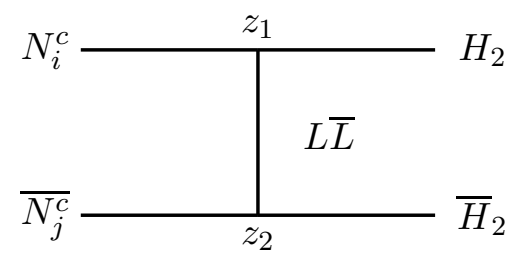

Figure 3.11: Pair annihilation of singlet neutrino superfields in configuration superspace. 


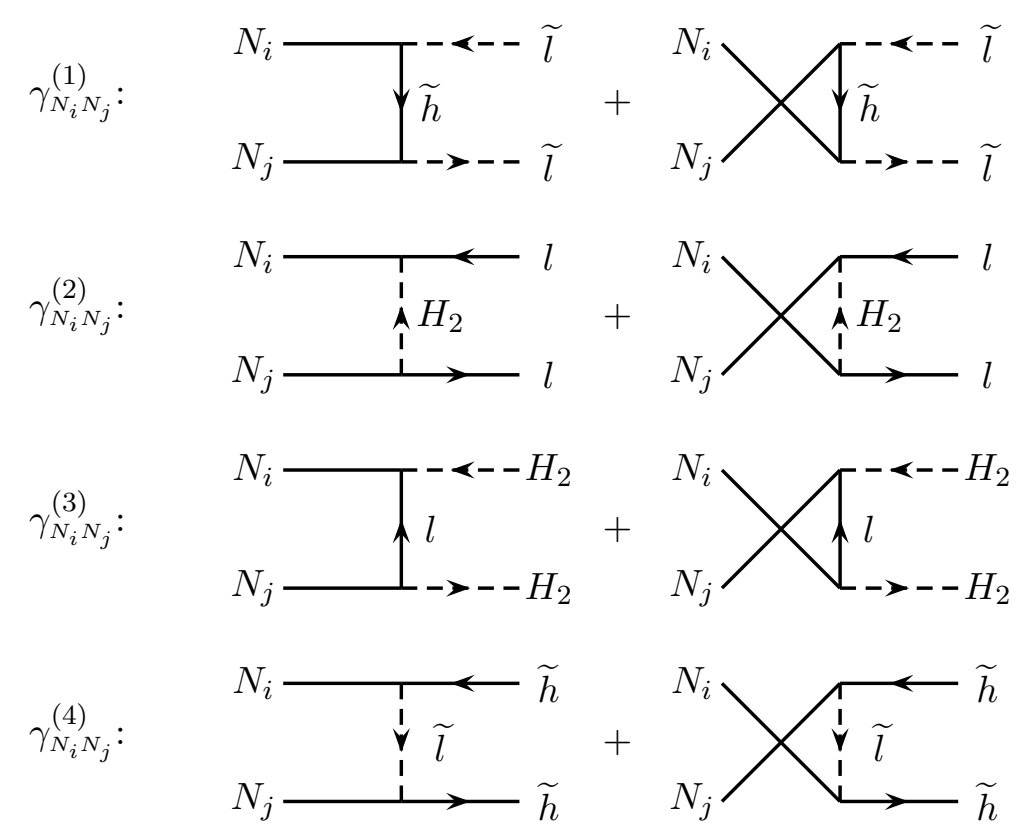

Figure 3.12: Neutrino pair annihilation.

Decomposing the superfield product into component fields with the help of eq. (B.57), we get the processes depicted in fig. 3.12 for the neutrinos

$$
\begin{array}{ll}
\gamma_{N_{i} N_{j}}^{(1)}=\gamma\left(N_{i}+N_{j} \leftrightarrow \widetilde{l}+\widetilde{l}^{\dagger}\right), & \gamma_{N_{i} N_{j}}^{(2)}=\gamma\left(N_{i}+N_{j} \leftrightarrow l+\bar{l}\right), \\
\gamma_{N_{i} N_{j}}^{(3)}=\gamma\left(N_{i}+N_{j} \leftrightarrow H_{2}+H_{2}^{\dagger}\right), & \gamma_{N_{i} N_{j}}^{(4)}=\gamma\left(N_{i}+N_{j} \leftrightarrow \widetilde{h}+\overline{\widetilde{h}}\right) .
\end{array}
$$

For the scalar neutrinos we have similar diagrams and additional contributions from quartic scalar couplings (cf. fig. 3.13). We have the following reaction densities

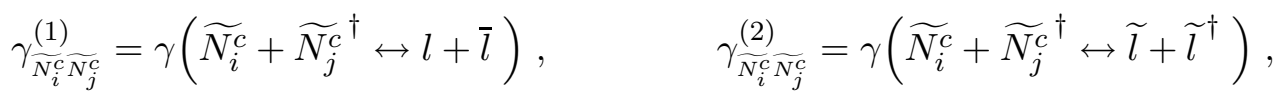

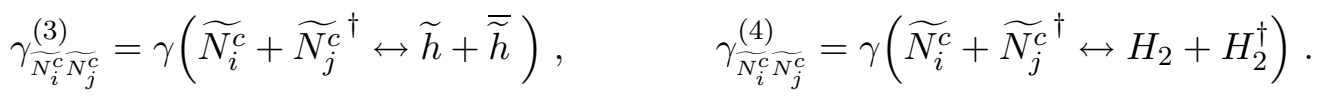

It is interesting to note that the contributions to $\gamma_{\widetilde{N_{i}^{c}} \widetilde{N_{j}^{c}}}^{(2)}$ and $\gamma_{\widetilde{N_{i}^{c}} \widetilde{(4)}}^{\left(N_{j}^{c}\right.}$ from the scalar potential are not contained in the superfield diagrams in fig. 3.11. They originate from the contribution of the auxiliary field $F_{H_{2}}$ (cf. eq. 3.16) to the decay diagram in fig. 3.1 a.

Finally, there are neutrino-sneutrino scattering processes (cf. fig. 3.14),

$$
\gamma_{N_{j} \widetilde{N}_{i}^{c}}^{(1)}=\gamma\left(\widetilde{N_{i}^{c}}+N_{j} \leftrightarrow \bar{l}+\widetilde{l}\right), \quad \gamma_{N_{j} \widetilde{N}_{i}^{c}}^{(2)}=\gamma\left(\widetilde{N_{i}^{c}}+N_{j} \leftrightarrow \widetilde{h}+H_{2}\right)
$$

Such diagrams also give neutrino-sneutrino transitions like $\widetilde{N_{i}^{c}}+l \leftrightarrow N_{j}+\widetilde{l}$. These processes transform neutrinos into sneutrinos and leptons into sleptons, i.e. they tend to balance out the 


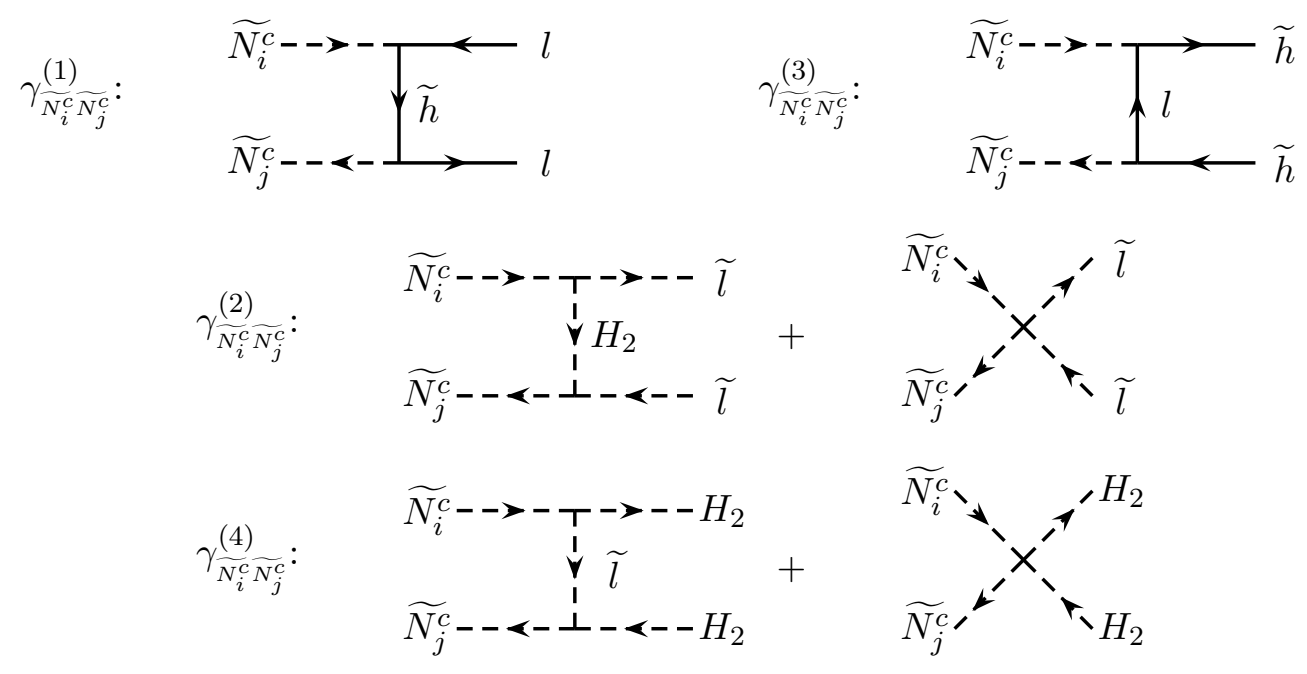

Figure 3.13: Sneutrino pair annihilation.

number densities of the fermions and their supersymmetric partners, but they cannot wash out any generated asymmetry. As we will see in the next chapter, the number densities of the neutrinos and the scalar neutrinos are already equal without taking into account these interactions, while the equality of the number densities of leptons and sleptons is ensured by MSSM-processes, which we are going to discuss in the next section. Finally, the dominant contributions to these neutrinosneutrino transitions come from inverse decays, decays and scatterings off a (s)top which we have already considered. Hence, we can neglect these additional processes.

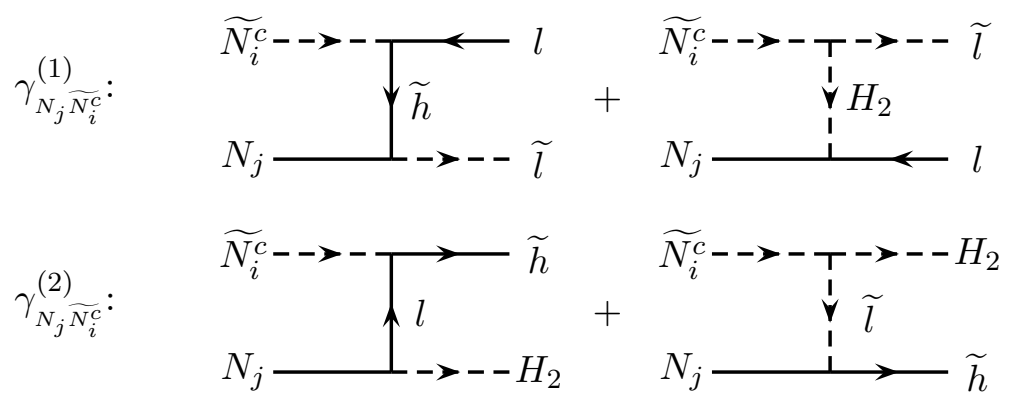

Figure 3.14: Neutrino-sneutrino scattering. 


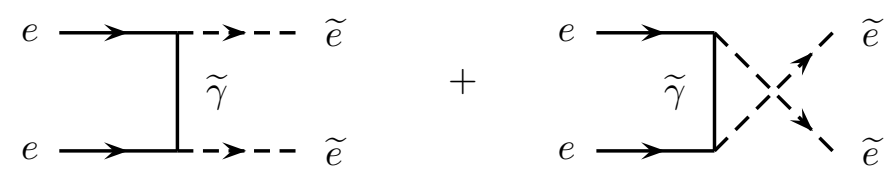

Figure 3.15: Example of a $L_{f}$ and $L_{s}$ violating MSSM process.

\subsection{MSSM Processes}

In the MSSM the fermionic lepton number $L_{f}$ and the lepton number stored in the scalar leptons $L_{s}$ are not separately conserved. There are processes transforming leptons into scalar leptons and vice versa. As an example we have considered the process $e+e \leftrightarrow \widetilde{e}+\widetilde{e}$ (cf. fig. 3.15). For large temperatures, i.e. $s \gg m_{\widetilde{\gamma}}^{2}$, the reduced cross section for this process is given by 37

$$
\hat{\sigma}_{\mathrm{MSSM}} \approx 4 \pi \alpha^{2}\left[\ln \left(\frac{s}{m_{\widetilde{\gamma}}^{2}}\right)-4\right] .
$$

This translates into the following reaction density

$$
\gamma_{\mathrm{MSSM}} \approx \frac{M_{1}^{4} \alpha^{2}}{4 \pi^{3}} \frac{1}{z^{4}}\left[\ln \left(\frac{4}{z^{2} a_{\widetilde{\gamma}}}\right)-2 \gamma_{\mathrm{E}}-3\right],
$$

where we have introduced the dimensionless squared photino mass

$$
a_{\widetilde{\gamma}}:=\left(\frac{m_{\widetilde{\gamma}}}{M_{1}}\right)^{2}
$$

These processes are in thermal equilibrium if the reaction rates are larger than the Hubble parameter $H$. This condition gives a very weak upper bound on the photino mass,

$$
m_{\widetilde{\gamma}} \lesssim 2.5 \times 10^{9} \mathrm{GeV}\left(\frac{T}{10^{10} \mathrm{GeV}}\right) \exp \left[-\frac{1}{412}\left(\frac{T}{10^{10} \mathrm{GeV}}\right)\right] .
$$

In the calculations we assume $m_{\widetilde{\gamma}}=100 \mathrm{GeV}$. 


\section{Chapter 4}

\section{Numerical Results}

Now that we have identified all the relevant processes we can write down the network of Boltzmann equations which governs the time evolution of the neutrino and sneutrino number densities and of the lepton asymmetry ${ }^{1}$. In this chapter we work out the parameter dependence of the generated baryon asymmetry by solving the Boltzmann equations, and we discuss the role of the different scattering and decay processes [17].

\subsection{The Boltzmann Equations}

The evolution of the neutrino number $Y_{N_{j}}$ as a function of the inverse dimensionless temperature $z=M_{1} / T$ is given by

$$
\begin{array}{r}
\frac{\mathrm{d} Y_{N_{j}}}{\mathrm{~d} z}=\frac{-z}{s H\left(M_{1}\right)}\left\{\left(\frac{Y_{N_{j}}}{Y_{N_{j}}^{\mathrm{eq}}}-1\right)\left[\gamma_{N_{j}}+4 \gamma_{t_{j}}^{(0)}+4 \gamma_{t_{j}}^{(1)}+4 \gamma_{t_{j}}^{(2)}+2 \gamma_{t_{j}}^{(3)}+4 \gamma_{t_{j}}^{(4)}\right]\right. \\
\left.+\sum_{i}\left[\left(\frac{Y_{N_{j}}}{Y_{N_{j}}^{\mathrm{eq}}} \frac{Y_{N_{i}}}{Y_{N_{i}}^{\mathrm{eq}}}-1\right) \sum_{k=1}^{4} \gamma_{N_{i} N_{j}}^{(k)}+\left(\frac{Y_{N_{j}}}{Y_{N_{j}}^{\mathrm{eq}}} \frac{Y_{i_{+}}}{Y_{N_{i}^{c}}^{\mathrm{eq}}}-2\right) \sum_{k=1}^{2} \gamma_{N_{j} \widetilde{N}_{i}^{c}}^{(k)}\right]\right\} .
\end{array}
$$

For the scalar neutrinos and their antiparticles it is convenient to use the sum and the difference of the particle numbers per comoving volume element as independent variables,

$$
Y_{j \pm}:=Y_{\widetilde{N_{j}^{c}}} \pm Y_{\widetilde{N}_{j}^{\dagger}}
$$

The Boltzmann equations for these quantities read

$$
\frac{\mathrm{d} Y_{j_{+}}}{\mathrm{d} z}=\frac{-z}{s H\left(M_{1}\right)}\left\{\left(\frac{Y_{j_{+}}}{Y_{\widehat{N_{j}^{c}}}^{\mathrm{eq}}}-2\right)\left(\gamma_{\bar{N}_{j}^{c}}^{(2)}+\gamma_{\bar{N}_{j}^{c}}^{(3)}+3 \gamma_{22_{j}}+2 \gamma_{t_{j}}^{(5)}+2 \gamma_{t_{j}}^{(6)}+2 \gamma_{t_{j}}^{(7)}+\gamma_{t_{j}}^{(8)}+2 \gamma_{t_{j}}^{(9)}\right)\right.
$$

\footnotetext{
${ }^{1}$ See app. D for a short review of kinetic theory in an expanding universe.
} 


$$
\begin{aligned}
& +\frac{1}{2} \frac{Y_{j_{-}}}{Y_{\widetilde{N}_{j}^{c}}^{\mathrm{eq}}} \frac{Y_{L_{s}}}{Y_{\widetilde{l}}^{\mathrm{eq}}}\left(\gamma_{22_{j}}-\gamma_{t_{j}}^{(8)}\right)+\frac{Y_{j_{-}}}{Y_{\widetilde{N}_{j}^{c}}^{\mathrm{eq}}} \frac{Y_{L_{f}}}{Y_{l}^{\mathrm{eq}}} \gamma_{t_{j}}^{(5)}
\end{aligned}
$$

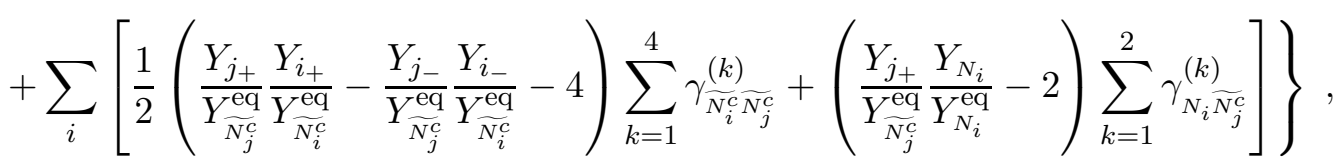

$$
\begin{aligned}
& \frac{\mathrm{d} Y_{j_{-}}}{\mathrm{d} z}=\frac{-z}{s H\left(M_{1}\right)}\left\{\frac{Y_{j_{-}}}{Y_{\bar{N}_{j}^{c}}^{\mathrm{eq}}}\left(\gamma_{\bar{N}_{j}^{c}}^{(2)}+\gamma_{\bar{N}_{j}^{c}}^{(3)}+3 \gamma_{22_{j}}+2 \gamma_{t_{j}}^{(5)}+2 \gamma_{t_{j}}^{(6)}+2 \gamma_{t_{j}}^{(7)}+\gamma_{t_{j}}^{(8)}+2 \gamma_{t_{j}}^{(9)}\right)\right. \\
& +\frac{Y_{L_{s}}}{Y_{\widetilde{l}}^{\mathrm{eq}}}\left[\gamma_{\widetilde{N_{j}^{c}}}^{(3)}-\frac{1}{2} \gamma_{\widetilde{N_{j}^{c}}}^{(2)}-2 \gamma_{t_{j}}^{(9)}-\frac{1}{2} \frac{Y_{j_{+}}}{Y_{\bar{N}_{j}^{c}}^{\mathrm{eq}}} \gamma_{t_{j}}^{(8)}+\left(2+\frac{1}{2} \frac{Y_{j_{+}}}{Y_{\bar{N}_{j}^{c}}^{\mathrm{eq}}}\right) \gamma_{22_{j}}\right] \\
& +\frac{Y_{L_{f}}}{Y_{l}^{\mathrm{eq}}}\left[\frac{1}{2} \gamma_{\widehat{N_{j}^{c}}}^{(2)}+2\left(\gamma_{t_{j}}^{(6)}+\gamma_{t_{j}}^{(7)}\right)+\frac{Y_{j_{+}}}{Y_{\mathrm{N}_{j}^{c}}^{\mathrm{eq}}} \gamma_{t_{j}}^{(5)}\right]
\end{aligned}
$$

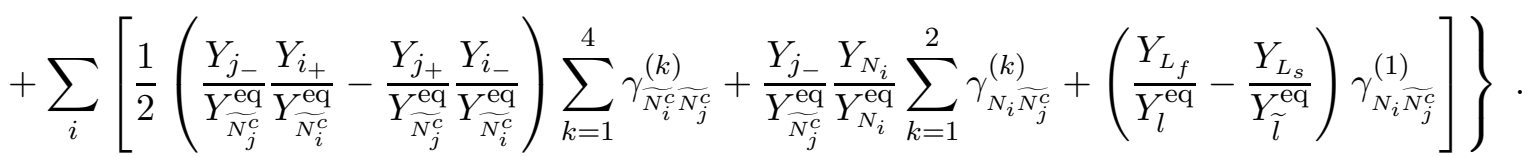

Furthermore, we have to discern the lepton asymmetry stored in the standard model particles $Y_{L_{f}}$ and the asymmetry $Y_{L_{s}}$ in the scalar leptons. Their evolution is governed by

$$
\begin{aligned}
& \frac{\mathrm{d} Y_{L_{f}}}{\mathrm{~d} z}=\frac{-z}{s H\left(M_{1}\right)}\left\{\sum_{j}\left[\left(\frac{1}{2} \frac{Y_{L_{f}}}{Y_{l}^{\mathrm{eq}}}+\varepsilon_{j}\right)\left(\frac{1}{2} \gamma_{N_{j}}+\gamma_{\widetilde{N_{j}^{c}}}^{(2)}\right)-\frac{1}{2} \varepsilon_{j}\left(\frac{Y_{N_{j}}}{Y_{N_{j}}^{\mathrm{eq}}} \gamma_{N_{j}}+\frac{Y_{j_{+}}}{Y_{\widetilde{N_{j}^{c}}}^{\mathrm{eq}}} \gamma_{\overline{N_{j}^{c}}}^{(2)}\right)+\frac{1}{2} \frac{Y_{j}}{Y_{\widetilde{N_{j}^{c}}}^{\mathrm{eq}}} \gamma_{\widetilde{N_{j}^{c}}}^{(2)}\right]\right. \\
& +\frac{Y_{L_{f}}}{Y_{l}^{\mathrm{eq}}}\left(\gamma_{A}^{\Delta L}+\gamma_{C}^{\Delta L}\right)+\frac{Y_{L_{s}}}{Y_{\widetilde{l}}^{\mathrm{eq}}}\left(\gamma_{B}^{\Delta L}-\gamma_{C}^{\Delta L}\right)+\left(\frac{Y_{L_{f}}}{Y_{l}^{\mathrm{eq}}}-\frac{Y_{L_{s}}}{Y_{\widetilde{l}}^{\mathrm{eq}}}\right) \gamma_{\mathrm{MSSM}} \\
& +\sum_{j}\left[\frac{Y_{L_{f}}}{Y_{l}^{\mathrm{eq}}}\left(\frac{Y_{N_{j}}}{Y_{N_{j}}^{\mathrm{eq}}} \gamma_{t_{j}}^{(3)}+\frac{Y_{j_{+}}}{Y_{\bar{N}_{j}^{c}}^{\mathrm{eq}}} \gamma_{t_{j}}^{(5)}+2 \gamma_{t_{j}}^{(4)}+2 \gamma_{t_{j}}^{(6)}+2 \gamma_{t_{j}}^{(7)}\right)+2 \frac{Y_{j_{-}}}{Y_{N_{j}^{c}}^{\mathrm{eq}}}\left(\gamma_{t_{j}}^{(5)}+\gamma_{t_{j}}^{(6)}+\gamma_{t_{j}}^{(7)}\right)\right] \\
& \left.+\sum_{i, j}\left(\frac{Y_{L_{f}}}{Y_{l}^{\mathrm{eq}}}-\frac{Y_{L_{s}}}{Y_{\widetilde{l}}^{\mathrm{eq}}}+\frac{Y_{N_{j}}}{Y_{N_{j}}^{\mathrm{eq}}} \frac{Y_{i_{-}}}{Y_{N_{i}^{c}}^{\mathrm{eq}}}\right) \gamma_{N_{j} \widetilde{N}_{i}^{c}}^{(1)}\right\} \\
& \frac{\mathrm{d} Y_{L_{s}}}{\mathrm{~d} z}=\frac{-z}{s H\left(M_{1}\right)}\left\{\sum _ { j } \left[\left(\frac{1}{2} \frac{Y_{L_{s}}}{Y_{\widetilde{l}}^{\mathrm{eq}}}+\varepsilon_{j}\right)\left(\frac{1}{2} \gamma_{N_{j}}+\gamma_{\overline{N_{j}^{c}}}^{(2)}\right)-\frac{1}{2} \varepsilon_{j}\left(\frac{Y_{N_{j}}}{Y_{N_{j}}^{\mathrm{eq}}} \gamma_{N_{j}}+\frac{Y_{j_{+}}}{Y_{\bar{N}_{j}^{c}}^{\mathrm{eq}}} \gamma_{\frac{N_{j}^{c}}{(2)}}\right)\right.\right.
\end{aligned}
$$

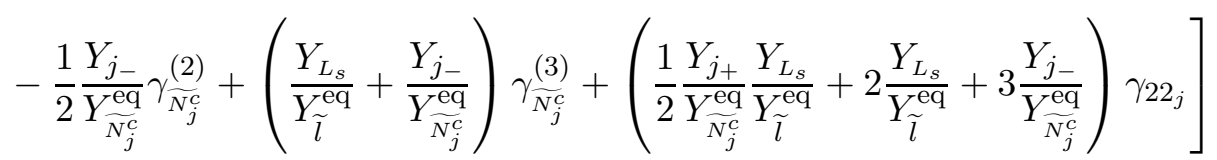




$$
\begin{aligned}
& +\frac{Y_{L_{s}}}{Y_{\widetilde{l}}^{\mathrm{eq}}}\left(\gamma_{A}^{\Delta L}+\gamma_{D}^{\Delta L}\right)+\frac{Y_{L_{f}}}{Y_{l}^{\mathrm{eq}}}\left(\gamma_{B}^{\Delta L}-\gamma_{C}^{\Delta L}\right)+\left(\frac{Y_{L_{s}}}{Y_{\widetilde{l}}^{\mathrm{eq}}}-\frac{Y_{L_{f}}}{Y_{l}^{\mathrm{eq}}}\right) \gamma_{\mathrm{MSSM}} \\
& +\sum_{j}\left[\frac{Y_{L_{s}}}{Y_{\widetilde{l}}^{\mathrm{eq}}}\left(2 \frac{Y_{N_{j}}}{Y_{N_{j}}^{\mathrm{eq}}} \gamma_{t_{j}}^{(0)}+\frac{1}{2} \frac{Y_{j_{+}}^{\mathrm{eq}}}{Y_{\widetilde{N}_{j}^{c}}^{(8)}} \gamma_{t_{j}}+2 \gamma_{t_{j}}^{(1)}+2 \gamma_{t_{j}}^{(2)}+2 \gamma_{t_{j}}^{(9)}\right)-\frac{Y_{j_{-}}}{Y_{\widetilde{N}_{j}^{c}}^{\mathrm{eq}}}\left(\gamma_{t_{j}}^{(8)}+2 \gamma_{t_{j}}^{(9)}\right)\right] \\
& \left.+\sum_{i, j}\left(\frac{Y_{L_{s}}}{Y_{\widetilde{l}}^{\mathrm{eq}}}-\frac{Y_{L_{f}}}{Y_{l}^{\mathrm{eq}}}-\frac{Y_{N_{j}}}{Y_{N_{j}}^{\mathrm{eq}}} \frac{Y_{i_{-}}}{Y_{\widetilde{N_{i}^{c}}}^{\mathrm{eq}}}\right) \gamma_{N_{j} \widetilde{N}_{i}^{c}}^{(1)}\right\},
\end{aligned}
$$

where we have introduced the following abbreviations for the lepton number violating scatterings mediated by a heavy (s)neutrino

$$
\begin{aligned}
& \gamma_{A}^{\Delta L}=2 \gamma_{N}^{(1)}+\gamma_{N}^{(3)}+\gamma_{N}^{(4)}+\gamma_{N}^{(6)}+\gamma_{N}^{(7)}+2 \gamma_{N}^{(12)}+\gamma_{N}^{(14)}, \\
& \gamma_{B}^{\Delta L}=\gamma_{N}^{(3)}+\gamma_{N}^{(4)}-\gamma_{N}^{(6)}-\gamma_{N}^{(7)}+\gamma_{N}^{(14)} \\
& \gamma_{C}^{\Delta L}=3 \gamma_{N}^{(9)}+\gamma_{N}^{(17)}+\gamma_{N}^{(18)}+6 \gamma_{N}^{(19)}, \\
& \gamma_{D}^{\Delta L}=4 \gamma_{N}^{(5)}+2 \gamma_{N}^{(8)}+8 \gamma_{N}^{(10)}+3 \gamma_{N}^{(9)}+4 \gamma_{N}^{(15)}+2 \gamma_{N}^{(16)}+\gamma_{N}^{(17)}+\gamma_{N}^{(18)}+6 \gamma_{N}^{(19)} .
\end{aligned}
$$

The numerical factors in front of the reaction densities arise due to the change in quantum numbers in the corresponding scattering, e.g. processes transforming leptons into sleptons appear with a relative minus sign in the Boltzmann equations for $Y_{L_{f}}$ and $Y_{L_{s}}$. Furthermore, any reaction density is multiplied by the number of different processes (cf. chapter 3) contributing independently to the Boltzmann equations.

This set of Boltzmann equations is valid for the most general case with arbitrary masses of the right-handed neutrinos. However, if the heavy neutrinos are mass degenerate, it is always possible to find a basis where the mass matrix $M$ and the Yukawa matrix $\lambda_{\nu}$ are diagonal, i.e. no asymmetry is generated. Therefore, one has to assume a mass hierarchy for the right-handed neutrinos, which in turn implies that the lepton number violating processes induced by the lightest right-handed neutrino are in thermal equilibrium as long as the temperature is higher than the mass of this neutrino. Hence, the lepton asymmetries generated in the decays of the heavier right-handed neutrinos are washed out and the asymmetry that we observe today must have been generated by the lightest right-handed neutrino. We will assume that the first generation neutrino $N_{1}$ is the lightest.

Hence, we will always neglect the heavier right-handed neutrinos as free particles. However, they have to be taken into account as intermediate states, since they give a substantial contribution to the effective lepton number violating processes at low energies. 
The fermionic part $Y_{L_{f}}$ of the generated lepton asymmetry will be transformed into a $(B-L)$ asymmetry by the action of sphalerons. But since MSSM processes like the one in section 3.6 enforce the relation

$$
Y_{L_{f}}=Y_{L_{s}}
$$

the total lepton asymmetry $Y_{L}=Y_{L_{f}}+Y_{L_{s}}$ will be proportional to the baryon asymmetry [38],

$$
Y_{B}=-\left(\frac{8 N_{f}+4 N_{H}}{22 N_{f}+13 N_{H}}\right) Y_{L}
$$

where $N_{f}$ is the number of quark-lepton families, and $N_{H}$ the number of Higgs doublets. In our model with $N_{f}=3$ and $N_{H}=2$ we have

$$
Y_{B}=-\frac{8}{23} Y_{L}
$$

From the observed baryon asymmetry

$$
Y_{B}=(0.6-1) \cdot 10^{-10}
$$

and eq. (4.11) we can infer the asymmetries that we have to generate,

$$
Y_{L_{f}}=Y_{L_{s}}=-(0.9-1.4) \cdot 10^{-10}
$$

The additional anomalous global symmetries in supersymmetric theories at high temperatures have no influence on these considerations, since they are broken well before the electroweak phase transition [39].

\subsection{The Generated Lepton Asymmetry}

Typical solutions of the Boltzmann equations are shown in fig. 4.1, where we have assumed a neutrino mass $M_{1}=10^{10} \mathrm{GeV}$, and a mass hierarchy of the form

$$
\begin{array}{ll}
a_{2}=10^{3}, & \left(m_{D}^{\dagger} m_{D}\right)_{22}=a_{2}\left(m_{D}^{\dagger} m_{D}\right)_{11}, \\
a_{3}=10^{6}, & \left(m_{D}^{\dagger} m_{D}\right)_{33}=a_{3}\left(m_{D}^{\dagger} m_{D}\right)_{11} .
\end{array}
$$

Furthermore, we have assumed a $C P$ asymmetry $\varepsilon_{1}=-10^{-6}$. The only difference between both figures lies in the choice of $\left(m_{D}^{\dagger} m_{D}\right)_{11}$,

$$
\widetilde{m}_{1}:=\frac{\left(m_{D}^{\dagger} m_{D}\right) 11}{M_{1}}= \begin{cases}10^{-4} \mathrm{eV} & \text { for fig. 4.1a } \\ 10^{-2} \mathrm{eV} & \text { for fig. 4.1 } \mathrm{b}\end{cases}
$$


(a)

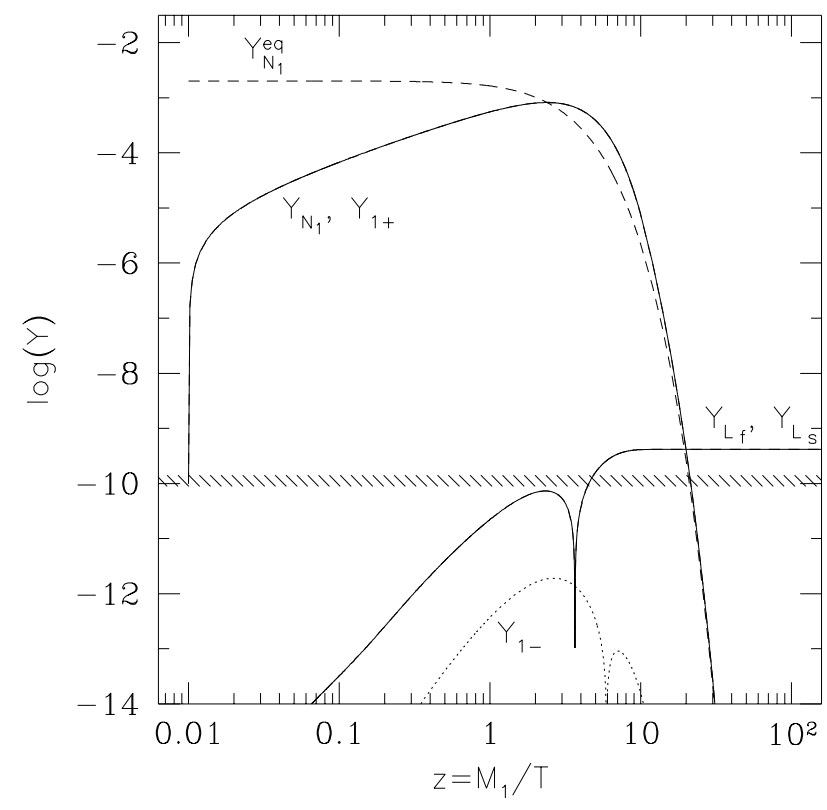

(b)

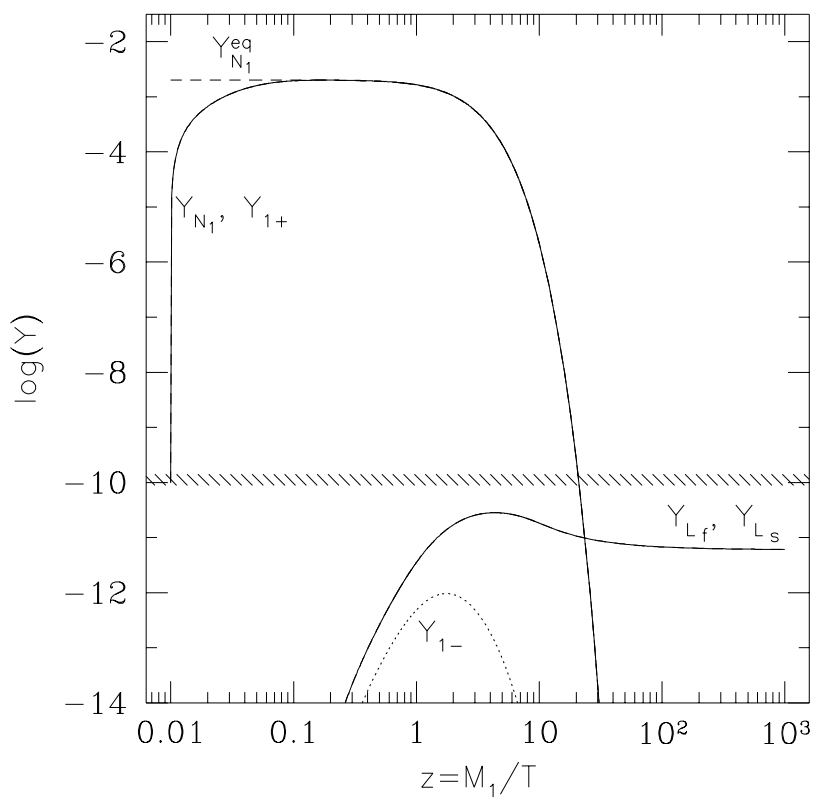

Figure 4.1: Typical solutions of the Boltzmann equations. The dashed line represents the equilibrium distribution for the neutrinos $N_{1}$ and the solid lines show the solutions for the (s)neutrino number and the absolute values of the asymmetries in $L_{f}$ and $L_{s}$, while the dotted line represents the absolute value of the scalar neutrino asymmetry $Y_{1-}$. The lines for $Y_{N_{1}}$ and $Y_{1+}$ and for the two asymmetries $Y_{L_{f}}$ and $Y_{L_{s}}$ cannot be distinguished, since they are lying one upon another. The hatched area shows the measured value (4.15).

Finally, as starting condition we have assumed that all the number densities vanish at high temperatures $T \gg M_{1}$, including the neutrino numbers $Y_{N_{1}}$ and $Y_{1+}$. As one can see, the Yukawa interactions are strong enough to create a substantial number of neutrinos and scalar neutrinos in fig. 4.1 a, even if $Y_{N_{1}}$ and $Y_{1+}$ do not reach their equilibrium values as long as $z<1$. However, the generated asymmetries

$$
Y_{L_{f}}=Y_{L_{s}}=-4 \cdot 10^{-10}
$$

are of the requested magnitude. On the other hand, in fig. 4.1 b the Yukawa interactions are much stronger, i.e. the neutrinos are driven into equilibrium rapidly at high temperatures. However, the large Yukawa couplings also increase the reaction rates for lepton number violating processes which can wash out a generated asymmetry, i.e. the final asymmetries are much smaller than in the previous case,

$$
Y_{L_{f}}=Y_{L_{s}}=-6 \cdot 10^{-12}
$$


In both cases a small scalar neutrino asymmetry $Y_{1-}$ is temporarily generated. However, $Y_{1-}$ is very small and has virtually no influence on the generated lepton asymmetries.

Usually it is assumed that one has a thermal population of right-handed neutrinos at high temperatures which decay at very low temperatures $T \ll M_{1}$ where one can neglect lepton number violating scatterings. Then the generated lepton asymmetry is proportional to the $C P$ asymmetry and the number of decaying neutrinos and sneutrinos [2],

$$
Y_{L} \approx \varepsilon_{1}\left[Y_{N_{1}}^{e q}\left(T \gg M_{1}\right)+Y_{1+}^{e q}\left(T \gg M_{1}\right)\right] \approx \frac{\varepsilon_{1}}{250} .
$$

With $\varepsilon_{1}=-10^{-6}$ this gives

$$
Y_{L_{f}}=Y_{L_{s}} \approx-2 \cdot 10^{-9}
$$

By comparison with eqs. (4.19) and (4.20) one sees that by assuming a thermal population of heavy neutrinos at high temperatures and neglecting the lepton number violating scatterings, one largely overestimates the generated lepton asymmetries.

A characteristic feature of the non-supersymmetric version of this baryogenesis mechanism is that the generated asymmetry does not depend on the neutrino mass $M_{1}$ and $\left(m_{D}^{\dagger} m_{D}\right)$ 11 separately but only on the ratio $\widetilde{m}_{1}$ [14]. To check if this is also the case in the supersymmetric scenario we have varied $\widetilde{m}_{1}$ while keeping all the other parameters fixed. In fig. 4.2 we have plotted the total lepton asymmetry $Y_{L}=Y_{L_{f}}+Y_{L_{s}}$ as a function of $\widetilde{m}_{1}$ for the right-handed neutrino masses $M_{1}=10^{12} \mathrm{GeV}, 10^{10} \mathrm{GeV}$ and $10^{8} \mathrm{GeV}$ and the $C P$ asymmetry $\varepsilon_{1}=-10^{-6}$.

The main difference between the supersymmetric and non-supersymmetric scenarios concerns the necessary production of the neutrinos at high temperatures. In the non-supersymmetric scenario the Yukawa interactions are too weak to account for this, i.e. additional interactions of the righthanded neutrinos have to be introduced. This is no longer the case here. The supersymmetric Yukawa interactions are much more important, and can produce a thermal population of righthanded neutrinos, i.e. the same vertices which are responsible for the generation of the asymmetry can also bring the neutrinos into thermal equilibrium at high temperatures. However, these lepton number violating processes will also erase a part of the generated asymmetry, hereby giving rise to the $\widetilde{m}_{1}$ dependence of the generated asymmetry which we shall discuss in detail.

First one sees that in the whole parameter range the generated asymmetry is much smaller than the naively expected value $4 \cdot 10^{-9}$. For low $\widetilde{m}_{1}$ the reason being that the Yukawa interactions are too weak to bring the neutrinos into equilibrium at high temperatures, like in fig. 4.1 a. For high $\widetilde{m}_{1}$ on the other hand, the lepton number violating scatterings wash out a large part of the generated asymmetry at temperatures $T<M_{1}$, like in fig. 4.1 b. Hence, the requested asymmetry 


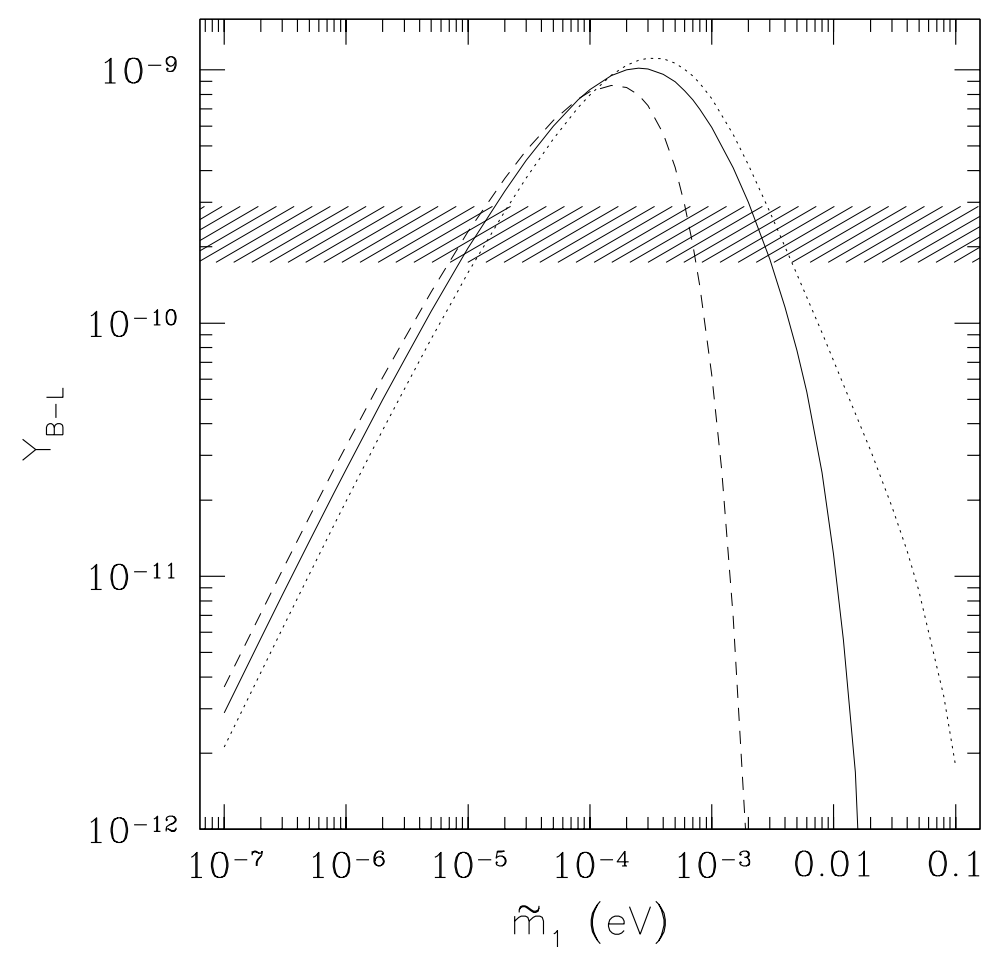

Figure 4.2: Generated $(B-L)$ asymmetry as a function of $\widetilde{m}_{1}$ for $M_{1}=10^{8}$ GeV (dotted line), $M_{1}=10^{10} \mathrm{GeV}$ (solid line) and $M_{1}=10^{12} \mathrm{GeV}$ (dashed line). The shaded area shows the measured value for the asymmetry.

can only be generated if $\widetilde{m}_{1}$ is larger than $\sim 10^{-5} \mathrm{eV}$ and smaller than $\sim 5 \cdot 10^{-3} \mathrm{eV}$, depending on the heavy neutrino mass $M_{1}$.

The asymmetry in fig. 4.2 depends almost only on $\widetilde{m}_{1}$ for small $\widetilde{m}_{1} \lesssim 10^{-4} \mathrm{eV}$, since in this region of parameter space the asymmetry depends mostly on the number of neutrinos generated at high temperatures, i.e. on the strength of the processes in which a right-handed neutrino can be generated or annihilated. The dominant reactions are decays, inverse decays and scatterings with a (s)top, which all give contributions proportional to $\widetilde{m}_{1}$ to the Boltzmann equations at high temperatures,

$$
\begin{aligned}
& \frac{-z}{s H\left(M_{1}\right)} \gamma_{N_{1}} \propto \widetilde{m}_{1}, \quad \frac{-z}{s H\left(M_{1}\right)} \gamma_{\tilde{N}_{1}^{c}}^{(2)} \propto \widetilde{m}_{1}, \quad \frac{-z}{s H\left(M_{1}\right)} \gamma_{\tilde{N}_{1}^{c}}^{(3)} \propto \widetilde{m}_{1}, \\
& \frac{-z}{s H\left(M_{1}\right)} \gamma_{22_{1}} \propto \widetilde{m}_{1}, \quad \frac{-z}{s H\left(M_{1}\right)} \gamma_{t_{1}}^{(i)}\left(T \gg M_{1}\right) \propto \widetilde{m}_{1} \text {. }
\end{aligned}
$$

For large $\widetilde{m}_{1} \gtrsim 10^{-4} \mathrm{eV}$ on the other hand, the neutrinos reach thermal equilibrium at high temperatures, i.e. the generated asymmetry depends mostly on the influence of the lepton number 
violating scatterings at temperatures $T \lesssim M_{1}$. In contrast to the relations of eq. (4.23) the lepton number violating processes mediated by a heavy neutrino behave like

$$
\frac{-z}{s H\left(M_{1}\right)} \gamma_{i}^{\Delta L} \propto M_{1} \sum_{j} \widetilde{m}_{j}^{2}, \quad i=A, \ldots, D
$$

at low temperatures. Hence, one expects that the generated asymmetry becomes smaller for growing neutrino mass $M_{1}$ and this is exactly what one observes in fig. 4.2.

Eq. (4.24) can also explain the small dependence of the asymmetry on the heavy neutrino mass $M_{1}$ for $\widetilde{m}_{1} \lesssim 10^{-4} \mathrm{eV}$. The inverse decay processes which take part in producing the neutrinos at high temperatures are $C P$ violating, i.e. they generate a lepton asymmetry at high temperatures. Due to the interplay of inverse decay processes and lepton number violating $2 \rightarrow 2$ scatterings this asymmetry has a different sign compared to the one generated in neutrino decays at low temperatures, i.e. the asymmetries will partially cancel each other, as one can see in the change of sign of the asymmetry in fig. 4.1 a. This cancellation can only be avoided if the asymmetry generated at high temperatures is washed out before the neutrinos decay. At high temperatures the lepton number violating scatterings behave like

$$
\frac{-z}{s H\left(M_{1}\right)} \gamma_{i}^{\Delta L} \propto M_{1} \sum_{j} a_{j} \widetilde{m}_{j}^{2}, \quad i=A, \ldots, D .
$$

Hence, the wash-out processes are more efficient for larger neutrino masses, i.e. the final asymmetry should grow with the neutrino mass $M_{1}$. The finally generated asymmetry is not affected by the stronger wash-out processes, since for small $\widetilde{m}_{1}$ the neutrinos decay late, where one can neglect the lepton number violating scatterings.

This change of sign in the asymmetry is not observed in fig. 4.1 b. Due to the larger $\tilde{m}_{1}$ value the neutrinos are brought into equilibrium at much higher temperatures, where decays and inverse decays are suppressed by a time dilatation factor, i.e. the (s)neutrinos are produced in CP invariant scatterings off a (s)top. 


\section{Chapter 5}

\section{$\mathrm{SO}(10)$ Unification and Neutrino Mixing}

In order to study the implications of leptogenesis for low-energy neutrino physics and leptonic flavour mixing we will assume a similar pattern of masses and mixings for the leptons and the quarks in this chapter 15, 17.

\subsection{Neutrino Masses and Mixings}

If we choose a basis where the Majorana mass matrix $M$ and the Dirac mass matrix $m_{l}$ for the charged leptons are diagonal with real and positive eigenvalues,

$$
m_{l}=\left(\begin{array}{ccc}
m_{e} & 0 & 0 \\
0 & m_{\mu} & 0 \\
0 & 0 & m_{\tau}
\end{array}\right), \quad M=\left(\begin{array}{ccc}
M_{1} & 0 & 0 \\
0 & M_{2} & 0 \\
0 & 0 & M_{3}
\end{array}\right),
$$

the Dirac mass matrix of the neutrinos can be written in the form

$$
m_{D}=V\left(\begin{array}{ccc}
m_{1} & 0 & 0 \\
0 & m_{2} & 0 \\
0 & 0 & m_{3}
\end{array}\right) U^{\dagger}
$$

where $V$ and $U$ are unitary matrices and the $m_{i}$ are real and positive.

Since the Majorana masses $M$ are assumed to be much larger than the Dirac masses $m_{D}$, we have 6 Majorana neutrinos as mass eigenstates [11]. In the weak eigenstate basis the mass matrix 
of the light neutrinos reads 40

$$
m_{\nu}^{\prime}=-m_{D} \frac{1}{M} m_{D}^{T}+\mathcal{O}\left(\frac{1}{M^{3}}\right) .
$$

It can be diagonalized by a unitary matrix $K$, i.e. the light mass eigenstates

$$
\nu_{i} \simeq\left(K^{\dagger} \nu_{\mathrm{L}}\right)_{i}+\left(\nu_{\mathrm{L}}^{\mathrm{C}} K\right)_{i}, \quad i=e, \mu, \tau
$$

have masses

$$
m_{\nu}=-K^{\dagger} m_{D} \frac{1}{M} m_{D}^{T} K^{*}+\mathcal{O}\left(\frac{1}{M^{3}}\right) \equiv\left(\begin{array}{ccc}
m_{\nu_{e}} & 0 & 0 \\
0 & m_{\nu_{\mu}} & 0 \\
0 & 0 & m_{\nu_{\tau}}
\end{array}\right)
$$

whereas the heavy neutrino mass eigenstates

$$
N_{i} \simeq \nu_{i, \mathrm{R}}+\nu_{i, \mathrm{R}}^{\mathrm{C}}, \quad i=1,2,3
$$

have masses

$$
m_{N}=M+\mathcal{O}\left(\frac{1}{M}\right)
$$

As we have seen in chapter 3, all the quantities relevant for baryogenesis, i.e. the decay widths, $C P$ asymmetries and scattering cross sections, depend only on the product $m_{D}^{\dagger} m_{D}$, where the mixing matrix $V$ drops out. On the other hand, the mixing matrix $K$ in the leptonic charged current depends on the parameters of both unitary matrices $U$ and $V$. Hence, leptonic mixing and $C P$ violation at high and low energies are to leading order independent, i.e. the $C P$ violation needed for baryogenesis does not allow to infer on $C P$ violating interactions of light leptons.

The mixing matrix $U$ can be parametrized by three mixing angles and six phases. Five of these phases can be factored out with the Gell-Mann matrices $\lambda_{i}$,

$$
U=\mathrm{e}^{i \gamma} \mathrm{e}^{i \lambda_{3} \alpha} \mathrm{e}^{i \lambda_{8} \beta} U_{1} \mathrm{e}^{i \lambda_{3} \sigma} \mathrm{e}^{i \lambda_{8} \tau}
$$

In analogy to the Cabibbo-Kobayashi-Maskawa (CKM) matrix for quarks the remaining matrix $U_{1}$ depends on three mixing angles and one phase. In unified theories based on $\mathrm{SO}(10)$ it is natural to assume a similar pattern of masses and mixings for leptons and quarks. This suggests the Wolfenstein parametrization [41] as an ansatz for $U_{1}$,

$$
U_{1}=\left(\begin{array}{ccc}
1-\frac{\lambda^{2}}{2} & \lambda & A \lambda^{3}(\rho-i \eta) \\
-\lambda & 1-\frac{\lambda^{2}}{2} & A \lambda^{2} \\
A \lambda^{3}(1-\rho-i \eta) & -A \lambda^{2} & 1
\end{array}\right)
$$


where $A$ and $|\rho+i \eta|$ are of order one, while the mixing parameter $\lambda$ is assumed to be small. For the Dirac masses $m_{i}, \mathrm{SO}(10)$ unification motivates a hierarchy like for the up-type quarks,

$$
m_{1}=b \lambda^{4} m_{3} \quad m_{2}=c \lambda^{2} m_{3} \quad b, c=\mathcal{O}(1) .
$$

We have mentioned in section 4.1 that we also need a hierarchy in the Majorana masses $M_{i}$ to get a non-vanishing lepton asymmetry. We choose a similar hierarchy as in eq. (5.10),

$$
M_{1}=B \lambda^{4} M_{3} \quad M_{2}=C \lambda^{2} M_{3} \quad B, C=\mathcal{O}(1) .
$$

Later on we will vary the parameters $B$ and $C$ to investigate different hierarchies for the righthanded neutrinos.

Diagonalizing the neutrino mass matrix (5.3) in powers of $\lambda$ yields the light neutrino masses

$$
\begin{aligned}
& m_{\nu_{e}}=\frac{b^{2}}{\left|C+\mathrm{e}^{4 i \alpha} B\right|} \lambda^{4} m_{\nu_{\tau}}+\mathcal{O}\left(\lambda^{6}\right), \\
& m_{\nu_{\mu}}=\frac{c^{2}\left|C+\mathrm{e}^{4 i \alpha} B\right|}{B C} \lambda^{2} m_{\nu_{\tau}}+\mathcal{O}\left(\lambda^{4}\right), \\
& m_{\nu_{\tau}}=\frac{m_{3}^{2}}{M_{3}}+\mathcal{O}\left(\lambda^{4}\right) .
\end{aligned}
$$

We will not discuss the masses of the light scalar neutrinos here, since they depend on unknown soft breaking terms.

In section 4.2 we have seen that the lepton asymmetry is largely determined by the mass parameter $\widetilde{m}_{1}$, which is given by

$$
\widetilde{m}_{1}=\frac{c^{2}+A^{2}|\rho+i \eta|^{2}}{B} \lambda^{2} m_{\nu_{\tau}}=\frac{C\left(c^{2}+A^{2}|\rho+i \eta|^{2}\right)}{c^{2}\left|C+\mathrm{e}^{4 i \alpha} B\right|} m_{\nu_{\mu}},
$$

i.e. $\widetilde{m}_{1}$ is of the same order as the $\nu_{\mu}$ mass. According to eq. (3.39) the $C P$ asymmetry in the decay of the lightest right-handed neutrino reads

$$
\varepsilon_{1}=\frac{3}{8 \pi} \frac{B A^{2}}{c^{2}+A^{2}|\rho+i \eta|^{2}} \lambda^{4} \frac{m_{3}^{2}}{v_{2}^{2}} \operatorname{Im}\left[(\rho-i \eta)^{2} \mathrm{e}^{i 2(\alpha+\sqrt{3} \beta)}\right]+\mathcal{O}\left(\lambda^{6}\right) .
$$

In the next section we will always assume maximal phases, i.e. we will set

$$
\varepsilon_{1}=-\frac{3}{8 \pi} \frac{B A^{2}|\rho+i \eta|^{2}}{c^{2}+A^{2}|\rho+i \eta|^{2}} \lambda^{4} \frac{m_{3}^{2}}{v_{2}^{2}}+\mathcal{O}\left(\lambda^{6}\right) .
$$

Hence, the lepton asymmetries that we are going to calculate may be viewed as upper bounds on the attainable asymmetries.

Like in the non-supersymmetric scenario a large value of the Yukawa-coupling $m_{3} / v_{2}$ will be preferred by this baryogenesis mechanism, since $\varepsilon_{1} \propto m_{3}^{2} / v_{2}^{2}$. This holds irrespective of our ansatz for neutrino mixings. 


\section{$5.2 \quad$ Numerical Results}

The neutrino masses (5.12)-(5.14) can be used to constrain the free parameters of our ansatz. The strongest hint for a non-vanishing neutrino mass being the solar neutrino deficit! we will fix the $\nu_{\mu}$ mass to the value preferred by the Mikheyev-Smirnov-Wolfenstein (MSW) solution [43],

$$
m_{\nu_{\mu}} \simeq 3 \cdot 10^{-3} \mathrm{eV}
$$

Hence the parameter $\widetilde{m}_{1}$, which is of the same order as $m_{\nu_{\mu}}$ according to eq. (5.15), will be in the interval allowed by fig. 4.2 .

The most obvious parameter choice is to take all $\mathcal{O}(1)$ parameters equal to one and to fix $\lambda$ to a similar value as the $\lambda$ parameter of the quark mixing matrix,

$$
\begin{gathered}
A=B=C=b=c=|\rho+i \eta| \simeq 1, \\
\lambda \simeq 0.1 .
\end{gathered}
$$

The $\nu_{\mu}$ mass in eqs. (5.13) and (5.18) then fixes the $\nu_{e}$ and $\nu_{\tau}$ masses,

$$
m_{\nu_{e}} \simeq 8 \cdot 10^{-6} \mathrm{eV}, \quad m_{\nu_{\tau}} \simeq 0.15 \mathrm{eV}
$$

and $\widetilde{m}_{1}$ reads

$$
\widetilde{m}_{1} \simeq 3 \cdot 10^{-3} \mathrm{eV}
$$

$\mathrm{SO}(10)$ unification suggests that the Dirac neutrino mass $m_{3}$ is equal to the top-quark mass,

$$
m_{3}=m_{t} \simeq 174 \mathrm{GeV}
$$

This leads to a large Majorana mass scale for the right-handed neutrinos,

$$
M_{3} \simeq 2 \cdot 10^{14} \mathrm{GeV} \quad \Rightarrow \quad M_{1} \simeq 2 \cdot 10^{10} \mathrm{GeV} \text { and } M_{2} \simeq 2 \cdot 10^{12} \mathrm{GeV},
$$

and eq. (5.17) gives the $C P$ asymmetry $\varepsilon_{1} \simeq-6 \cdot 10^{-6}$. Integration of the Boltzmann equations yields the $(B-L)$ asymmetry (cf. fig. 5.1 a)

$$
Y_{B-L} \simeq 1 \cdot 10^{-9}
$$

which is of the correct order of magnitude. It is interesting to note that in the non-supersymmetric scenario one has $Y_{B-L} \simeq 9 \cdot 10^{-10}$ for the same choice of parameters.

\footnotetext{
${ }^{1}$ For a review and references, see [42].
} 
(a)

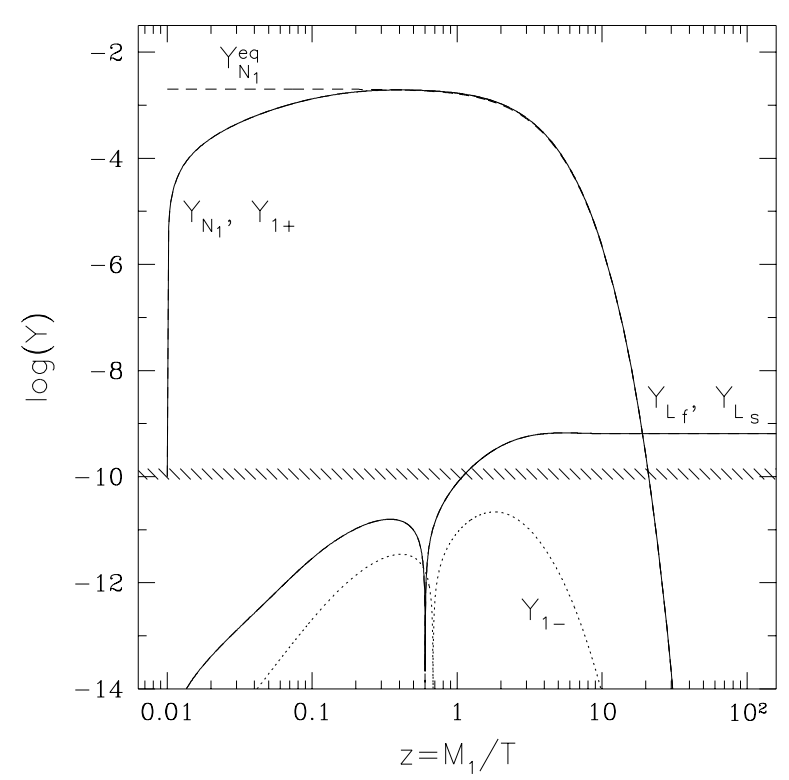

(b)

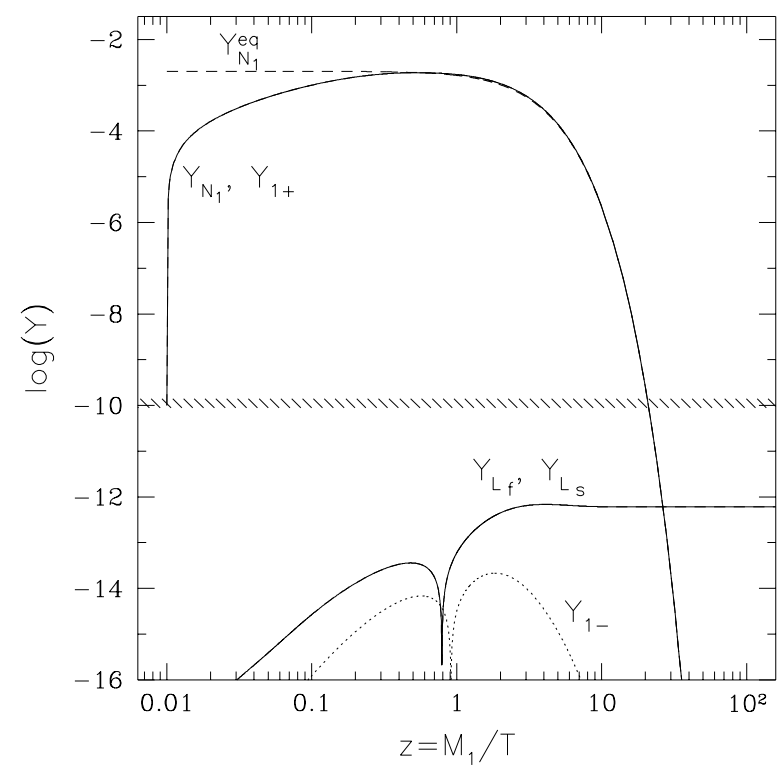

Figure 5.1: Generated asymmetry if one assumes a similar pattern of masses and mixings for the leptons and the quarks. In both figures we have $\lambda=0.1$ and $m_{3}=m_{t}$ (a) and $m_{3}=m_{b}$ (b).

Our assumption (5.23), $m_{3} \simeq m_{t}$ led to a large Majorana mass scale $M_{3}$ in eq. (5.24). To check the sensitivity of our result for the baryon asymmetry on this choice, we have envisaged a lower Dirac mass scale

$$
m_{3}=m_{b} \simeq 4.5 \mathrm{GeV},
$$

while keeping all other parameters in eqs. (5.19) and (5.20) fixed. The assumed $\nu_{\mu}$ mass (5.18) then yields a much lower value for the Majorana mass scale,

$$
M_{3} \simeq 1 \cdot 10^{11} \mathrm{GeV} \quad \Rightarrow \quad M_{1} \simeq 1 \cdot 10^{7} \mathrm{GeV} \text { and } M_{2} \simeq 1 \cdot 10^{9} \mathrm{GeV}
$$

while the light neutrino masses (5.21) remain unchanged. The $C P$ asymmetry $\varepsilon_{1} \simeq-4 \cdot 10^{-9}$ becomes very small. Consequently, the generated baryon asymmetry (cf. fig. 5.1]b)

$$
Y_{B-L} \simeq 1 \cdot 10^{-12}
$$

is too small by two orders of magnitude. We can conclude that high values for both masses $m_{3}$ and $M_{3}$ are preferred. This suggests that $(B-L)$ is already broken at the unification scale $\Lambda_{\mathrm{GUT}} \sim 10^{16} \mathrm{GeV}$, without any intermediate scale of symmetry breaking, which is natural in $\mathrm{SO}(10)$ unification. Alternatively, a Majorana mass scale of the order of $10^{12}$ to $10^{14} \mathrm{GeV}$ can also 


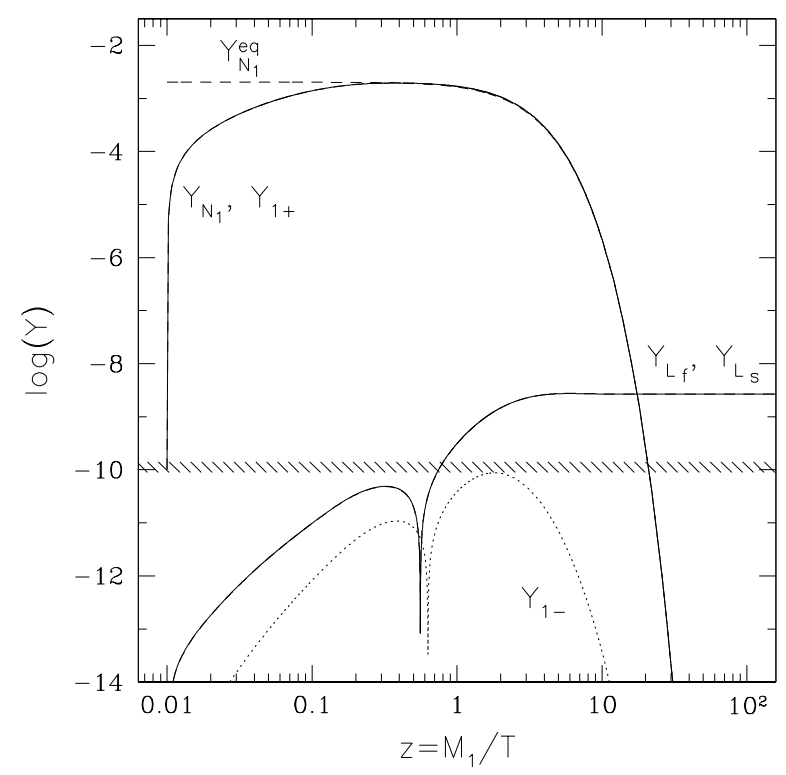

Figure 5.2: Solution of the Boltzmann equations suggested by the atmospheric neutrino problem.

be generated radiatively if $\mathrm{SO}(10)$ is broken into $\mathrm{SU}(5)$ at some high scale between $10^{16} \mathrm{GeV}$ and the Planck-scale, and SU(5) is subsequently broken into the MSSM gauge group at the usual GUT scale $\sim 10^{16} \mathrm{GeV}$ 44].

Such a Majorana mass scale naturally leads to a baryogenesis scale $M_{1} \sim 10^{10} \mathrm{GeV}$. As discussed in section 4.2 and as one can see in fig. 5.1, the neutrinos can be brought into equilibrium at temperatures slightly above their mass, i.e. this scenario requires a reheating temperature $\sim 10^{10} \mathrm{GeV}$ at the end of inflation. This is well compatible with the constraints on the reheating temperature from the gravitino problem [45].

It is interesting to note that the $\nu_{\tau}$ mass in eq. (5.21) has got almost the value suggested by the atmospheric neutrino problem 46]

$$
m_{\nu_{\tau}} \approx 7 \cdot 10^{-2} \mathrm{eV}
$$

if one assumes that the anomalous $\mu$ to $e$ ratio produced by atmospheric neutrinos is due to oscillations from $\nu_{\mu}$ to $\nu_{\tau}$, and when the $\nu_{\mu}$ mass is again given by the MSW value in eq. (5.18). If we then use eq. (5.29) to fix the $\nu_{\tau}$ mass, the Dirac mass scale (5.23) determines the Majorana mass scale

$$
M_{3} \simeq 4 \cdot 10^{14} \mathrm{GeV}
$$




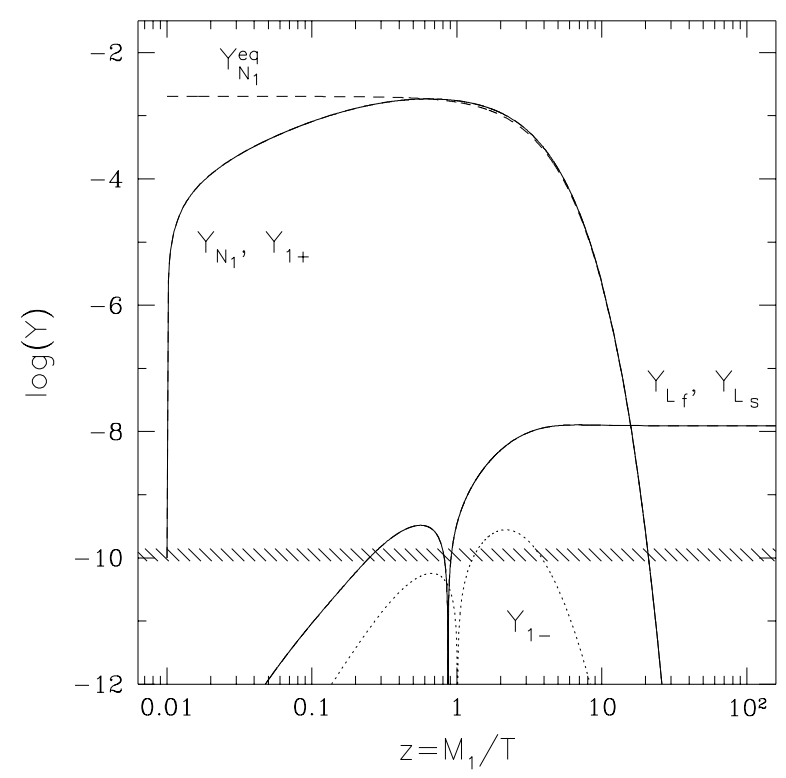

Figure 5.3: Generated lepton asymmetry if one assumes a similar mass hierarchy for the righthanded neutrinos and the down-type quarks.

The ratio of the $\nu_{\tau}$ and $\nu_{\mu}$ masses then yields the mixing parameter

$$
\lambda=0.15
$$

if the $\mathcal{O}(1)$ parameters are given by eq. (5.19). The remaining neutrino masses read

$$
M_{1} \simeq 2 \cdot 10^{11} \mathrm{GeV}, \quad M_{2} \simeq 9 \cdot 10^{12} \mathrm{GeV}, \quad m_{\nu_{e}} \simeq 2 \cdot 10^{-5} \mathrm{eV} .
$$

Consequently, we again get a large $C P$ asymmetry $\varepsilon_{1} \simeq-3 \cdot 10^{-5}$, and a large $(B-L)$ asymmetry (cf. fig. 5.2)

$$
Y_{B-L} \simeq 5 \cdot 10^{-9}
$$

This shows that the parameters required to explain the anomalous results of neutrino experiments by neutrino oscillations also predict a baryon asymmetry of the correct order of magnitude, although the large mixing angle which seems to be required to solve the atmospheric neutrino problem is difficult to accommodate within our small-mixing ansatz.

Up to now we have always assumed a mass hierarchy for the heavy Majorana neutrinos like for the up-type quarks. Alternatively, one can assume a weaker hierarchy, like for the down-type quarks by choosing

$$
B=10 \quad \text { and } \quad C=3 .
$$


Keeping the other parameters in eqs. (5.19) and (5.20) unchanged fixes the $\nu_{e}$ and $\nu_{\tau}$ masses,

$$
m_{\nu_{e}} \simeq 5 \cdot 10^{-6} \mathrm{eV}, \quad m_{\nu_{\tau}} \simeq 0.7 \mathrm{eV},
$$

and the mass parameter $\widetilde{m}_{1}$,

$$
\widetilde{m}_{1} \simeq 1 \cdot 10^{-3} \mathrm{eV}
$$

Choosing the Dirac mass scale (5.23) we get a large Majorana mass scale

$$
M_{3} \simeq 4 \cdot 10^{13} \mathrm{GeV} \quad \Rightarrow \quad M_{1} \simeq 4 \cdot 10^{10} \mathrm{GeV} \text { and } M_{2} \simeq 10^{12} \mathrm{GeV}
$$

From eq. (5.17) one obtains the $C P$ asymmetry $\varepsilon_{1} \simeq-6 \cdot 10^{-5}$. The corresponding solutions of the Boltzmann equations are shown in fig. 5.3. The final $(B-L)$ asymmetry,

$$
Y_{B-L} \simeq 2 \cdot 10^{-8}
$$

is much larger than requested, but this value can always be lowered by adjusting the unknown phases. Hence, the possibility to generate a lepton asymmetry does not depend on the special form of the mass hierarchy assumed for the right-handed neutrinos.

In the non-supersymmetric scenario one finds for the same parameter choice

$$
Y_{B-L} \simeq 2 \cdot 10^{-8}
$$

Hence, when comparing the supersymmetric and the non-supersymmetric scenario, one sees that the larger $C P$ asymmetry in the former and the additional contributions from the sneutrino decays are compensated by the wash-out processes which are stronger than in the latter. 


\section{Conclusions}

We have analysed in detail the generation of a cosmological baryon asymmetry by out-of-equilibrium decays of heavy right-handed Majorana neutrinos and their scalar partners in a supersymmetric extension of the Standard Model. By developing a resummed perturbative expansion in flavour non-diagonal self-energies, we could show how self-energy contributions to $C P$ asymmetries in heavy neutrino decays are consistently taken into account.

We have discussed all the decays and scattering processes relevant for leptogenesis, and by solving the Boltzmann equations we have shown that, in order to be consistent, one has to pay attention to two phenomena which can hamper the generation of a lepton asymmetry.

First, one has to take into consideration lepton number violating scatterings mediated by a heavy neutrino or its scalar partner. These processes, which are usually neglected, can wash out a large part of the asymmetry if the Yukawa couplings of the right-handed neutrinos become too large.

On the other hand, the neutrinos have to be brought into thermal equilibrium at high temperatures. We could show that for this purpose it is not necessary to assume additional interactions of the right-handed neutrinos in our theory, since the Yukawa interactions can be sufficiently strong to produce a thermal population of heavy neutrinos at high temperatures, while still being weak enough to prevent the final asymmetry from being washed out.

The observed baryon asymmetry can be obtained without any fine tuning of parameters if one assumes a similar pattern of mixings and Dirac masses for the neutrinos and the up-type quarks. Then the generated asymmetry is related to the $\nu_{\mu}$ mass, and fixing this mass to the value preferred by the MSW-solution to the solar neutrino problem leads to a baryon asymmetry of the requested order, provided $(B-L)$ is broken at the unification scale, as suggested by supersymmetric $\mathrm{SO}(10)$ unification. The baryon asymmetry is generated at a scale of approximately $10^{10} \mathrm{GeV}$, which looks promising with respect to the gravitino problem. 
In supersymmetric theories there are further possible sources of a $(B-L)$ asymmetry, e.g. it may be possible to combine inflation with leptogenesis by using a right-handed scalar neutrino as the inflaton (cf. refs. [47]). In this connection, possible constraints on the neutrino masses and on the reheating temperature from lepton number violating processes at low temperatures require further studies.

Furthermore, it should be studied to which extent a "primordial" baryon asymmetry, i.e. an asymmetry generated during or shortly after reheating, is affected if right-handed neutrinos come into equilibrium after reheating. This may yield interesting constraints on Yukawa interactions of first generation leptons, since an asymmetry in right-handed electrons might be protected from being washed-out if right-handed electrons decouple from the thermal plasma [48].

Finally, as discussed in the first chapter, one has to separate "on-shell" and "off-shell" contributions to lepton number violating scatterings, in order to be able to describe the generation of a baryon asymmetry in terms of Boltzmann equations. To avoid this separation and treat these contributions simultaneously, one has to go beyond the semi-classical approximation realized in the Boltzmann equations by constructing a complete nonequilibrium quantum kinetic theory. Although such a theory has not been realized up to now, quantum corrections to the Boltzmann equations have recently been investigated in refs. 49.]. 


\section{Appendix A}

\section{One-Loop Integrals}

We summarize some standard formulae for dimensionally regularized one-loop integrals in Minkowski space. We follow the notation of refs. [50], although we use a metric $g_{\mu \nu}=(+,-,-,-)$ (cf. e.g. ref. [51]).

\section{A.1 One-Point Function}

In $n=4-2 \epsilon$ dimensions the scalar one-point function is defined by

$$
A\left(m_{1}\right)=\frac{\mu^{4-n}}{i \pi^{2}} \int d^{n} k \frac{1}{k^{2}-m_{1}^{2}+i \varepsilon} .
$$

In the limit $\epsilon \rightarrow 0, A\left(m_{1}\right)$ is given by

$$
A\left(m_{1}\right)=m_{1}^{2}\left(\Delta-\ln \left(\frac{m_{1}^{2}}{\mu^{2}}\right)+1\right)
$$

where the UV-divergence is contained in

$$
\Delta=\frac{1}{\epsilon}-C+\ln (4 \pi)
$$

and $C=0.577216$ is Euler's constant. Note that the massless tadpole $A(0)$ vanishes in dimensional regularization, and that $A\left(m_{1}\right)$ has no absorptive contribution

$$
\operatorname{Im}\left[A\left(m_{1}\right)\right]=0 .
$$

\section{A.2 Two-Point Functions}

Three different two-point integrals can occur

$$
B_{0}\left(p_{1}^{2}, m_{1}, m_{2}\right)=\frac{\mu^{4-n}}{i \pi^{2}} \int d^{n} k \frac{1}{\left(k^{2}-m_{1}^{2}+i \varepsilon\right)\left[\left(k+p_{1}\right)^{2}-m_{2}^{2}+i \varepsilon\right]}
$$




$$
\begin{aligned}
B_{\mu}\left(p_{1}^{2}, m_{1}, m_{2}\right) & =\frac{\mu^{4-n}}{i \pi^{2}} \int d^{n} k \frac{k_{\mu}}{\left(k^{2}-m_{1}^{2}+i \varepsilon\right)\left[\left(k+p_{1}\right)^{2}-m_{2}^{2}+i \varepsilon\right]} \\
B_{\mu \nu}\left(p_{1}^{2}, m_{1}, m_{2}\right) & =\frac{\mu^{4-n}}{i \pi^{2}} \int d^{n} k \frac{k_{\mu} k_{\nu}}{\left(k^{2}-m_{1}^{2}+i \varepsilon\right)\left[\left(k+p_{1}\right)^{2}-m_{2}^{2}+i \varepsilon\right]}
\end{aligned}
$$

Lorentz covariance of the integrals allows to decompose the tensor integrals into tensors constructed from the external momentum $p_{1}$, and the metric tensor $g_{\mu \nu}$

$$
\begin{aligned}
B_{\mu}\left(p_{1}^{2}, m_{1}, m_{2}\right) & =p_{1, \mu} B_{1}\left(p_{1}^{2}, m_{1}, m_{2}\right) \\
B_{\mu \nu}\left(p_{1}^{2}, m_{1}, m_{2}\right) & =p_{1, \mu} p_{1, \nu} B_{21}\left(p_{1}^{2}, m_{1}, m_{2}\right)+g_{\mu \nu} B_{22}\left(p_{1}^{2}, m_{1}, m_{2}\right) .
\end{aligned}
$$

Using the Feynman parametrization, one can derive an integral representation for $B_{0}$,

$$
B_{0}\left(p_{1}^{2}, m_{1}, m_{2}\right)=\Delta-\int_{0}^{1} d x \ln \left(\frac{x^{2} p_{1}^{2}-x\left(p_{1}^{2}+m_{1}^{2}-m_{2}^{2}\right)+m_{1}^{2}-i \varepsilon}{\mu^{2}}\right)+\mathcal{O}(n-4),
$$

which yields the following useful identities in the limit $n \rightarrow 4$

$$
\begin{aligned}
& B_{0}\left(p_{1}^{2}, 0,0\right)=\Delta-\ln \left(\frac{\left|p_{1}^{2}\right|}{\mu^{2}}\right)+2+i \pi \theta\left(p_{1}^{2}\right) \\
& B_{0}(0,0, m)=B_{0}(0, m, 0)=\Delta-\ln \left(\frac{m^{2}}{\mu^{2}}\right)+1=\frac{1}{m^{2}} A\left(m^{2}\right) .
\end{aligned}
$$

Contracting eqs. A.6)-(A.9) with $p_{1, \mu}$ and $g_{\mu \nu}$ yields a set of coupled linear equations, which determine the scalar coefficients $B_{1}, B_{21}$ and $B_{22}$,

$$
\begin{aligned}
B_{1}\left(p_{1}^{2}, m_{1}, m_{2}\right)= & \frac{1}{2 p_{1}^{2}}\left[A\left(m_{1}\right)-A\left(m_{2}\right)+\left(m_{2}^{2}-m_{1}^{2}-p_{1}^{2}\right) B_{0}\left(p_{1}^{2}, m_{1}, m_{2}\right)\right] \\
= & -\frac{1}{2 \epsilon}+\mathrm{UV} \text {-finite parts, } \\
B_{21}\left(p_{1}^{2}, m_{1}, m_{2}\right)= & \frac{1}{3 p_{1}^{2}}\left[A\left(m_{2}\right)-m_{1}^{2} B_{0}\left(p_{1}^{2}, m_{1}, m_{2}\right)\right. \\
& \left.-2\left(p_{1}^{2}+m_{1}^{2}-m_{2}^{2}\right) B_{1}\left(p_{1}^{2}, m_{1}, m_{2}\right)-\frac{1}{2}\left(m_{1}^{2}+m_{2}^{2}-\frac{1}{3} p_{1}^{2}\right)\right] \\
= & \frac{1}{3 \epsilon}+\mathrm{UV} \text {-finite parts, } \\
B_{22}\left(p_{1}^{2}, m_{1}, m_{2}\right)= & \frac{1}{6}\left[A\left(m_{2}\right)+2 m_{1}^{2} B_{0}\left(p_{1}^{2}, m_{1}, m_{2}\right)\right. \\
& \left.+\left(p_{1}^{2}+m_{1}^{2}-m_{2}^{2}\right) B_{1}\left(p_{1}^{2}, m_{1}, m_{2}\right)+m_{1}^{2}+m_{2}^{2}-\frac{1}{3} p_{1}^{2}\right] \\
= & -\frac{1}{12 \epsilon}\left(p_{1}^{2}-3 m_{1}^{2}-3 m_{2}^{2}\right)+\mathrm{UV} \text {-finite parts. }
\end{aligned}
$$


In the case of equal or vanishing masses one has

$$
\begin{aligned}
B_{1}\left(p_{1}^{2}, m, m\right) & =-\frac{1}{2} B_{0}\left(p_{1}^{2}, m, m\right), \\
B_{21}\left(p_{1}^{2}, 0,0\right) & =\frac{1}{3}\left[B_{0}\left(p_{1}^{2}, 0,0\right)+\frac{1}{6}\right], \\
B_{22}\left(p_{1}^{2}, 0,0\right) & =\frac{-p_{1}^{2}}{12}\left[B_{0}\left(p_{1}^{2}, 0,0\right)+\frac{2}{3}\right] .
\end{aligned}
$$

\section{A.3 Three-Point Functions}

In general one has four different three-point functions

$$
\begin{aligned}
C_{0}\left(p_{1}^{2}, p_{2}^{2}, m_{1}, m_{2}, m_{3}\right) & =\frac{\mu^{4-n}}{i \pi^{2}} \int d^{n} k \frac{1}{D_{m_{1}} D_{m_{2}}\left(p_{1}\right) D_{m_{3}}\left(p_{1}, p_{2}\right)}, \\
C_{\mu}\left(p_{1}^{2}, p_{2}^{2}, m_{1}, m_{2}, m_{3}\right) & =\frac{\mu^{4-n}}{i \pi^{2}} \int d^{n} k \frac{k_{\mu}}{D_{m_{1}} D_{m_{2}}\left(p_{1}\right) D_{m_{3}}\left(p_{1}, p_{2}\right)}, \\
C_{\mu \nu}\left(p_{1}^{2}, p_{2}^{2}, m_{1}, m_{2}, m_{3}\right) & =\frac{\mu^{4-n}}{i \pi^{2}} \int d^{n} k \frac{k_{\mu} k_{\nu}}{D_{m_{1}} D_{m_{2}}\left(p_{1}\right) D_{m_{3}}\left(p_{1}, p_{2}\right)}, \\
C_{\mu \nu \rho}\left(p_{1}^{2}, p_{2}^{2}, m_{1}, m_{2}, m_{3}\right) & =\frac{\mu^{4-n}}{i \pi^{2}} \int d^{n} k \frac{k_{\mu} k_{\nu} k_{\rho}}{D_{m_{1}} D_{m_{2}}\left(p_{1}\right) D_{m_{3}}\left(p_{1}, p_{2}\right)},
\end{aligned}
$$

where we have introduced the following abbreviations for inverse propagators

$$
\begin{aligned}
D_{m_{1}} & =k^{2}-m_{1}^{2}+i \varepsilon \\
D_{m_{2}}\left(p_{1}\right) & =\left(k+p_{1}\right)^{2}-m_{2}^{2}+i \varepsilon, \\
D_{m_{3}}\left(p_{1}, p_{2}\right) & =\left(k+p_{1}+p_{2}\right)^{2}-m_{3}^{2}+i \varepsilon .
\end{aligned}
$$

Like for the two point-functions, Lorentz covariance of the integrals suggests the following tensor decomposition of the tensor three-point functions

$$
\begin{aligned}
C_{\mu}\left(p_{1}^{2}, p_{2}^{2}, m_{1}, m_{2}, m_{3}\right)= & p_{1, \mu} C_{11}+p_{2, \mu} C_{12}, \\
C_{\mu \nu}\left(p_{1}^{2}, p_{2}^{2}, m_{1}, m_{2}, m_{3}\right)= & p_{1, \mu} p_{1, \nu} C_{21}+p_{2, \mu} p_{2, \nu} C_{22} \\
& +\left(p_{1} p_{2}\right)_{(\mu \nu)} C_{23}+g_{\mu \nu} C_{24}, \\
C_{\mu \nu \rho}\left(p_{1}^{2}, p_{2}^{2}, m_{1}, m_{2}, m_{3}\right)= & p_{1, \mu} p_{1, \nu} p_{1, \rho} C_{31}+p_{2, \mu} p_{2, \nu} p_{2, \rho} C_{32} \\
& +\left(p_{2} p_{1} p_{1}\right)_{(\mu \nu \rho)} C_{33}+\left(p_{1} p_{2} p_{2}\right)_{(\mu \nu \rho)} C_{34} \\
& +\left(p_{1} g\right)_{(\mu \nu \rho)} C_{35}+\left(p_{2} g\right)_{(\mu \nu \rho)} C_{36},
\end{aligned}
$$


where we have used the following abbreviations for index symmetrizations

$$
\begin{aligned}
\left(p_{1} p_{2}\right)_{(\mu \nu)} & =p_{1, \mu} p_{2, \nu}+p_{1, \nu} p_{2, \mu} \\
\left(p_{1} p_{2} p_{2}\right)_{(\mu \nu \rho)} & =p_{1, \mu} p_{2, \nu} p_{2, \rho}+p_{2, \mu} p_{1, \nu} p_{2, \rho}+p_{2, \mu} p_{2, \nu} p_{1, \rho} \\
\left(p_{1} g\right)_{(\mu \nu \rho)} & =p_{1, \mu} g_{\nu \rho}+p_{1, \nu} g_{\mu \rho}+p_{1, \rho} g_{\mu \nu} .
\end{aligned}
$$

The form factors $C_{i j}$ can be related to the scalar functions $A, B_{0}$ and $C_{0}$ by contracting the definitions (A.23)-(A.25) and A.29)-(A.31) with external momenta $p_{1}, p_{2}$ and the metric $g^{\mu \nu}$.

In our calculation we only need three-point functions with two vanishing masses $\left(m_{2}=m_{3}=0\right)$, and two light-like momenta $p_{1}^{2}=0$ and $\left(p_{1}+p_{2}\right)^{2}=0$. Then $C_{12}$ and $C_{11}$ read

$$
\begin{aligned}
& C_{12}\left(p_{1}, p_{2}, m, 0,0\right)=\frac{1}{2 p_{1} \cdot p_{2}}\left[B_{0}(0, m, 0)-B_{0}\left(p_{2}^{2}, 0,0\right)-m^{2} C_{0}\left(p_{1}, p_{2}, m, 0,0\right)\right] \\
& C_{11}\left(p_{1}, p_{2}, m, 0,0\right)=2 C_{12}\left(p_{1}, p_{2}, m, 0,0\right)
\end{aligned}
$$

and the imaginary parts of $C_{0}$ and $C_{12}$ are given by

$$
\begin{aligned}
\operatorname{Im}\left[C_{0}\left(p_{1}, p_{2}, m, 0,0\right)\right] & =-\frac{\pi \theta\left(p_{2}^{2}\right)}{p_{2}^{2}} \ln \left(1+\frac{p_{2}^{2}}{m^{2}}\right) \\
\operatorname{Im}\left[C_{12}\left(p_{1}, p_{2}, m, 0,0\right)\right] & =\frac{\pi \theta\left(p_{2}^{2}\right)}{p_{2}^{2}}\left[1-\frac{m^{2}}{p_{2}^{2}} \ln \left(1+\frac{p_{2}^{2}}{m^{2}}\right)\right] .
\end{aligned}
$$




\section{Appendix B}

\section{Spinor Notation and Conventions}

We will use the conventions of ref. [30] with flat space-time metric $g_{\mu \nu}=(+,-,-,-)$. Greek indices $\alpha, \beta, \dot{\alpha}$ and $\dot{\beta}$ run from one to two and denote two-component Weyl spinors, while all other Greek letters denote Lorentz-indices.

\section{B.1 Weyl Spinors}

Two-component spinors $\psi$ and $\bar{\psi}$ transform under the $\left(\frac{1}{2}, 0\right)$ and $\left(0, \frac{1}{2}\right)$ representations of the Lorentz group $\mathrm{SO}(1,3)$. Matrix representations are given by the universal covering group $\operatorname{SL}(2, \mathbb{C})$ of $\mathrm{SO}(1,3)$, i.e. under a Lorentz transformation $M \in \mathrm{SL}(2, \mathbb{C})$ the Weyl spinors transform like

$$
\begin{aligned}
\psi_{\alpha}^{\prime}=M_{\alpha}{ }^{\beta} \psi_{\beta}, & \bar{\psi}_{\dot{\alpha}}=M_{\dot{\alpha}}^{*} \dot{\beta} \bar{\psi}_{\dot{\beta}}, \\
\psi^{\prime \alpha}=M^{-1}{ }_{\beta}^{\alpha} \psi^{\beta}, & {\overline{\psi^{\prime}}}^{\dot{\alpha}}=\left(M^{*}\right)^{-1} \dot{\beta} \bar{\psi}^{\dot{\beta}} .
\end{aligned}
$$

The Pauli matrices $\sigma_{\alpha \dot{\alpha}}{ }^{\mu}$ form a basis of $\operatorname{SL}(2, \mathbb{C})$,

$$
\begin{array}{rlrl}
\sigma^{0} & =\left(\begin{array}{cc}
1 & 0 \\
0 & 1
\end{array}\right), & \sigma^{1}=\left(\begin{array}{cc}
0 & 1 \\
1 & 0
\end{array}\right), \\
\sigma^{2}=\left(\begin{array}{cc}
0 & -i \\
i & 0
\end{array}\right), & \sigma^{3}=\left(\begin{array}{cc}
1 & 0 \\
0 & -1
\end{array}\right) .
\end{array}
$$

Spinor indices can be raised and lowered using the antisymmetric $\varepsilon$-tensors

$$
\varepsilon_{\alpha \beta}=\left(\begin{array}{cc}
0 & -1 \\
1 & 0
\end{array}\right), \quad \varepsilon^{\alpha \beta}=\left(\begin{array}{cc}
0 & 1 \\
-1 & 0
\end{array}\right)
$$




$$
\varepsilon_{\dot{\alpha} \dot{\beta}}=\left(\begin{array}{cc}
0 & -1 \\
1 & 0
\end{array}\right), \quad \quad \varepsilon^{\dot{\alpha} \dot{\beta}}=\left(\begin{array}{cc}
0 & 1 \\
-1 & 0
\end{array}\right)
$$

i.e. one has

$$
\begin{array}{ll}
\psi^{\alpha}=\varepsilon^{\alpha \beta} \psi_{\beta}, & \psi_{\alpha}=\varepsilon_{\alpha \beta} \psi^{\beta}, \\
\psi^{\dot{\alpha}}=\varepsilon^{\dot{\alpha} \dot{\beta}} \psi_{\dot{\beta}}, & \psi_{\dot{\alpha}}=\varepsilon_{\dot{\alpha} \dot{\beta}} \psi^{\dot{\beta}} .
\end{array}
$$

Correspondingly, spinor indices of Pauli matrices can be raised and lowered,

$$
\bar{\sigma}^{\mu \dot{\alpha} \alpha}=\varepsilon^{\dot{\alpha} \dot{\beta}} \varepsilon^{\alpha \beta} \sigma_{\beta \dot{\beta}}^{\mu},
$$

i.e. the matrices $\bar{\sigma}^{\mu}$ are given by

$$
\bar{\sigma}^{0}=\sigma^{0}, \quad \bar{\sigma}^{1,2,3}=-\sigma^{1,2,3} .
$$

The Pauli matrices fulfil

$$
\begin{aligned}
\left(\sigma^{\mu} \bar{\sigma}^{\nu}+\sigma^{\nu} \bar{\sigma}^{\mu}\right)_{\alpha}^{\beta} & =2 g^{\mu \nu} \delta_{\alpha}{ }^{\beta}, \\
\left(\bar{\sigma}^{\mu} \sigma^{\nu}+\bar{\sigma}^{\nu} \sigma^{\mu}\right)_{\dot{\beta}}^{\dot{\alpha}} & =2 g^{\mu \nu} \delta_{\dot{\beta}}^{\dot{\alpha}}, \\
\operatorname{Tr} \sigma^{\mu} \bar{\sigma}^{\nu} & =2 g^{\mu \nu}, \\
\sigma_{\alpha \dot{\alpha}}{ }^{\mu} \bar{\sigma}_{\mu}{ }^{\dot{\beta} \beta} & =2 \delta_{\alpha}{ }^{\beta} \delta_{\dot{\alpha}} \dot{\beta}
\end{aligned}
$$

Products of two-component spinors are defined such that

$$
\begin{gathered}
\psi \chi \equiv \psi^{\alpha} \chi_{\alpha}=-\psi_{\alpha} \chi^{\alpha}=\chi^{\alpha} \psi_{\alpha}=\chi \psi, \\
\bar{\psi} \bar{\chi} \equiv \bar{\psi}_{\dot{\alpha}} \bar{\chi}^{\dot{\alpha}}=-\bar{\psi}^{\dot{\alpha}} \bar{\chi}_{\dot{\alpha}}=\bar{\chi}_{\dot{\alpha}} \bar{\psi}^{\dot{\alpha}}=\bar{\chi} \bar{\psi} \\
(\chi \psi)^{\dagger}=\left(\chi^{\alpha} \psi_{\alpha}\right)^{\dagger}=\bar{\psi}_{\dot{\alpha}} \bar{\chi}^{\dot{\alpha}}=\bar{\psi} \bar{\chi} .
\end{gathered}
$$

Products of Weyl-spinors involving Pauli matrices read

$$
\begin{aligned}
\chi \sigma^{\mu} \bar{\psi} & =-\bar{\psi} \bar{\sigma}^{\mu} \chi \\
\left(\chi \sigma^{\mu} \bar{\psi}\right)^{\dagger} & =\psi \sigma^{\mu} \bar{\chi}, \\
\chi \sigma^{\mu} \bar{\sigma}^{\nu} \psi & =\psi \sigma^{\nu} \bar{\sigma}^{\mu} \chi, \\
\left(\chi \sigma^{\mu} \bar{\sigma}^{\nu} \psi\right)^{\dagger} & =\bar{\psi} \bar{\sigma}^{\nu} \sigma^{\mu} \bar{\chi}, \\
(\psi \lambda) \bar{\chi}_{\dot{\alpha}} & =\frac{1}{2}\left(\lambda \sigma^{\mu} \bar{\chi}\right)\left(\psi \sigma_{\mu}\right)_{\dot{\alpha}} .
\end{aligned}
$$


Furthermore, when computing superfield products (cf. app. B.3), one can take advantage of the relations

$$
\begin{aligned}
\theta^{\alpha} \theta^{\beta} & =-\frac{1}{2} \varepsilon^{\alpha \beta} \theta^{2}, \\
\theta_{\alpha} \theta_{\beta} & =\frac{1}{2} \varepsilon_{\alpha \beta} \theta^{2}, \\
\bar{\theta}^{\dot{\alpha}} \bar{\theta}^{\dot{\beta}} & =\frac{1}{2} \varepsilon^{\dot{\alpha} \dot{\beta}} \bar{\theta}^{2}, \\
\bar{\theta}_{\dot{\alpha}} \bar{\theta} \dot{\beta} & =-\frac{1}{2} \varepsilon \dot{\alpha} \dot{\beta} \bar{\theta}^{2}, \\
\theta \sigma^{\mu} \bar{\theta} \theta \sigma^{\nu} \bar{\theta} & =\frac{1}{2} \theta^{2} \bar{\theta}^{2} g^{\mu \nu} .
\end{aligned}
$$

\section{B.2 Four-Component Spinors}

We define the Dirac $\gamma$-matrices as

$$
\gamma^{\mu}=\left(\begin{array}{cc}
0 & \sigma^{\mu} \\
\bar{\sigma}^{\mu} & 0
\end{array}\right), \quad \gamma^{5}=i \gamma^{0} \gamma^{1} \gamma^{2} \gamma^{3}=\left(\begin{array}{cc}
-\mathbb{1} & 0 \\
0 & \mathbb{1}
\end{array}\right),
$$

obeying

$$
\begin{aligned}
& \left\{\gamma^{\mu}, \gamma^{\nu}\right\}=2 g^{\mu \nu} \\
& \left\{\gamma^{5}, \gamma^{\mu}\right\}=0 \\
& \left(\gamma^{5}\right)^{2}=1
\end{aligned}
$$

As usual, $\gamma^{0}$ intertwines the $\gamma^{\mu}$ representation of the Dirac algebra with the equivalent hermitian conjugated representation $\gamma^{\mu \dagger}$

$$
\begin{aligned}
& \gamma^{0} \gamma^{\mu} \gamma^{0}=\gamma^{\mu \dagger} \\
& \gamma^{0} \gamma^{5} \gamma^{0}=-\gamma^{5^{\dagger}}=-\gamma^{5}
\end{aligned}
$$

This representation of the $\gamma$-matrices can be used to relate Weyl spinors to the more familiar four-component spinors. A Dirac spinor $\Psi_{\mathrm{D}}$ consists of two Weyl spinors

$$
\Psi_{\mathrm{D}}=\left(\begin{array}{c}
\chi_{\alpha} \\
\bar{\lambda}^{\dot{\alpha}}
\end{array}\right)
$$

i.e. its hermitian conjugate reads

$$
\bar{\Psi}_{\mathrm{D}} \equiv \Psi_{\mathrm{D}}^{\dagger} \gamma^{0}=\left(\lambda^{\alpha}, \bar{\chi}_{\dot{\alpha}}\right)
$$


The chiral projectors read

$$
P_{\mathrm{R}}=\frac{1}{2}\left(1+\gamma^{5}\right)=\left(\begin{array}{ll}
0 & 0 \\
0 & \mathbb{1}
\end{array}\right), \quad P_{\mathrm{L}}=\frac{1}{2}\left(1-\gamma^{5}\right)=\left(\begin{array}{ll}
\mathbb{1} & 0 \\
0 & 0
\end{array}\right)
$$

i.e. for the Dirac spinor (B.34) one has

$$
\begin{array}{ll}
P_{\mathrm{L}} \Psi_{\mathrm{D}}=\chi_{\alpha}, & P_{\mathrm{R}} \Psi_{\mathrm{D}}=\bar{\lambda}^{\dot{\alpha}}, \\
\bar{\Psi}_{\mathrm{D}} P_{\mathrm{L}}=\lambda^{\alpha}, & \bar{\Psi}_{\mathrm{D}} P_{\mathrm{R}}=\bar{\chi}_{\dot{\alpha}} .
\end{array}
$$

The charge conjugation matrix $C$ is defined by

$$
C=-i \gamma^{2} \gamma^{0}=\left(\begin{array}{cc}
\varepsilon_{\alpha \beta} & 0 \\
0 & \varepsilon^{\dot{\alpha} \dot{\beta}}
\end{array}\right)
$$

and it fulfils the following useful identities

$$
C^{T}=C^{\dagger}=C^{-1}=-C, \quad C^{2}=-1
$$

$C$ intertwines the $\gamma^{\mu}$ and $-\gamma_{\mu}^{T}$ representations of the Dirac algebra

$$
\begin{aligned}
& C \gamma_{\mu} C^{-1}=-\gamma_{\mu}^{T}, \\
& C \gamma_{5} C^{-1}=\gamma_{5}^{T}
\end{aligned}
$$

The charge conjugated Dirac spinor (B.34) then reads

$$
\Psi_{\mathrm{D}}^{\mathrm{C}} \equiv C \bar{\Psi}_{\mathrm{D}}^{T}=\left(\begin{array}{c}
\lambda_{\alpha} \\
\bar{\chi}^{\dot{\alpha}}
\end{array}\right)
$$

i.e. one has

$$
\bar{\Psi}_{\mathrm{D}}=\Psi_{\mathrm{D}}^{\mathrm{C} T} C, \quad \Psi_{\mathrm{D}}=C{\overline{\Psi_{\mathrm{D}}^{\mathrm{C}}}}^{T}, \quad \overline{\Psi_{\mathrm{D}}^{\mathrm{C}}}=\Psi_{\mathrm{D}}^{T} C
$$

On the other hand, a Majorana spinor $\Psi_{\mathrm{M}}$ contains only one Weyl spinor

$$
\Psi_{\mathrm{M}}=\left(\begin{array}{c}
\chi_{\alpha} \\
\bar{\chi}^{\dot{\alpha}}
\end{array}\right)
$$

i.e. eq. (B.43) immediately implies

$$
\Psi_{\mathrm{M}}^{\mathrm{C}}=\Psi_{\mathrm{M}}
$$


In Lagrange densities we can switch from Weyl spinors to four-component spinors and vice versa by means of the following relations

$$
\begin{aligned}
\overline{\Psi_{1}} P_{\mathrm{L}} \Psi_{2} & =\lambda_{1} \chi_{2}, \\
\overline{\Psi_{1}} P_{\mathrm{R}} \Psi_{2} & =\overline{\chi_{1}} \overline{\lambda_{2}}, \\
\overline{\Psi_{1}} P_{\mathrm{L}} \gamma^{\mu} \Psi_{2} & =\lambda_{1} \sigma^{\mu} \overline{\lambda_{2}}=-\overline{\lambda_{2}} \bar{\sigma}^{\mu} \lambda_{1}, \\
\overline{\Psi_{1}} P_{\mathrm{R}} \gamma^{\mu} \Psi_{2} & =\overline{\chi_{1}} \bar{\sigma}^{\mu} \chi_{2}=-\chi_{2} \sigma^{\mu} \overline{\chi_{1}}, \\
\overline{\Psi_{1}} P_{\mathrm{L}} \gamma^{\mu} \gamma^{\nu} \Psi_{2} & =\lambda_{1} \sigma^{\mu} \bar{\sigma}^{\nu} \chi_{2}=\chi_{2} \sigma^{\nu} \bar{\sigma}^{\mu} \lambda_{1}, \\
\overline{\Psi_{1}} P_{\mathrm{R}} \gamma^{\mu} \gamma^{\nu} \Psi_{2} & =\overline{\chi_{1}} \bar{\sigma}^{\mu} \sigma^{\nu} \overline{\lambda_{2}}=\overline{\lambda_{2}} \bar{\sigma}^{\nu} \sigma^{\mu} \overline{\chi_{1}},
\end{aligned}
$$

where $\Psi_{i}(i=1,2)$ is a generic Dirac spinor consisting of two Weyl spinors $\chi_{i}$ and $\overline{\lambda_{i}}$ (cf. eq. (B.34) $)$.

\section{B.3 Superfield Products}

Since products of chiral superfields are again chiral, these products can be conveniently computed in the $y$-basis (2.31). For the products of two or three chiral superfields $\Phi_{i}$ one finds

$$
\begin{aligned}
\Phi_{i}(y) \Phi_{j}(y) & =A_{i}(y) A_{j}(y)+\sqrt{2} \theta\left[\psi_{i}(y) A_{j}(y)+A_{i}(y) \psi_{j}(y)\right] \\
+ & \theta^{2}\left[A_{i}(y) F_{j}(y)+A_{j}(y) F_{i}(y)-\psi_{i}(y) \psi_{j}(y)\right] \\
\Phi_{i}(y) \Phi_{j}(y) \Phi_{k}(y)=A_{i}(y) A_{j}(y) A_{k}(y) & \\
+ & \sqrt{2} \theta\left[\psi_{i}(y) A_{j}(y) A_{k}(y)+\psi_{j}(y) A_{k}(y) A_{i}(y)+\psi_{k}(y) A_{i}(y) A_{j}(y)\right] \\
+ & \theta^{2}\left[F_{i}(y) A_{j}(y) A_{k}(y)+F_{j}(y) A_{k}(y) A_{i}(y)+F_{k}(y) A_{i}(y) A_{j}(y)\right. \\
& \left.-\psi_{i}(y) \psi_{j}(y) A_{k}(y)-\psi_{j}(y) \psi_{k}(y) A_{i}(y)-\psi_{k}(y) \psi_{i}(y) A_{j}(y)\right] .
\end{aligned}
$$

On the other hand, the product of a chiral superfield $\Phi_{j}$ and an antichiral superfield $\Phi_{i}^{\dagger}$ is neither chiral nor antichiral. In terms of the variables $x^{\mu}, \theta$ and $\bar{\theta}$ it reads

$$
\begin{aligned}
\bar{\Phi}_{i}(x) \Phi_{j}(x) & =A_{i}^{*}(x) A_{j}(x)+\sqrt{2}\left(\theta \psi_{j}(x)\right) A_{i}^{*}(x)+\sqrt{2}\left(\bar{\theta} \overline{\psi_{i}}(x)\right) A_{j}(x) \\
+ & \theta^{2} A_{i}^{*}(x) F_{j}(x)+\bar{\theta}^{2} A_{j}(x) F_{i}^{*}(x) \\
+ & \theta^{\alpha} \bar{\theta}^{\dot{\alpha}}\left[-i{\sigma_{\alpha \dot{\alpha}}}^{\mu}\left(A_{i}^{*}(x) \partial_{\mu} A_{j}(x)-A_{j}(x) \partial_{\mu} A_{i}^{*}(x)\right)-2 \overline{\psi_{i}}(x) \psi_{j_{\alpha}}\right]
\end{aligned}
$$




$$
\begin{aligned}
+\theta^{2} \bar{\theta}^{\dot{\alpha}}\left[\frac{-i}{\sqrt{2}} \sigma_{\alpha \dot{\alpha}}^{\mu}\left(A_{i}^{*}(x) \partial_{\mu} \psi_{j}^{\alpha}(x)-\psi_{j}^{\alpha}(x) \partial_{\mu} A_{i}^{*}(x)\right)-\sqrt{2} F_{j}(x) \overline{\psi_{i}} \cdot(x)\right] \\
+\bar{\theta}^{2} \theta^{\alpha}\left[\frac{i}{\sqrt{2}} \sigma_{\alpha \dot{\alpha}}^{\mu}\left(\bar{\psi}_{i}^{\dot{\alpha}}(x) \partial_{\mu} A_{j}(x)-A_{j}(x) \partial_{\mu} \bar{\psi}_{i}^{\dot{\alpha}}(x)\right)+\sqrt{2} F_{i}^{*}(x) \psi_{j \alpha}(x)\right] \\
+\theta^{2} \bar{\theta}^{2}\left[F_{i}^{*}(x) F_{j}(x)-\frac{1}{4} A_{i}^{*}(x) \square A_{j}(x)-\frac{1}{4} A_{j}(x) \square A_{i}^{*}(x)\right. \\
\left.+\frac{1}{2}\left(\partial_{\mu} A_{i}^{*}(x)\right)\left(\partial^{\mu} A_{j}(x)\right)-\frac{i}{2} \partial_{\mu} \overline{\psi_{i}}(x) \bar{\sigma}^{\mu} \psi_{j}(x)+\frac{i}{2} \overline{\psi_{i}}(x) \bar{\sigma}^{\mu} \partial_{\mu} \psi_{j}(x)\right] .
\end{aligned}
$$

Superspace integration discussed in Chapter 2 will project out $F$-terms, i.e. terms proportional to $\theta^{2}$ in chiral superfields and the $D$-term (proportional to $\theta^{2} \bar{\theta}^{2}$ ) in eq. (B.55). These are the terms which can be used to construct SUSY-invariant actions since they transform into a spacetime derivative under SUSY transformations.

When computing $2 \rightarrow 2$ scatterings in superspace one has to evaluate products of four chiral or anti-chiral superfields, with or without covariant derivatives acting on them. In our calculations we need the following two products, which can be computed by successively using eqs. (B.53) and (B.55)

$$
\begin{aligned}
& \int d^{2} \theta d^{2} \bar{\theta} \Phi_{r}\left(x_{1}, \theta, \bar{\theta}\right) \Phi_{s}\left(x_{1}, \theta, \bar{\theta}\right) \frac{1}{4} \frac{D_{2}^{2}}{\square_{2}} \Phi_{i}\left(x_{2}, \theta, \bar{\theta}\right) \Phi_{j}\left(x_{2}, \theta, \bar{\theta}\right)= \\
&=\left[A_{r}\left(x_{1}\right) F_{s}\left(x_{1}\right)+A_{s}\left(x_{1}\right) F_{r}\left(x_{1}\right)-\psi_{r}\left(x_{1}\right) \psi_{s}\left(x_{1}\right)\right] A_{i}\left(x_{2}\right) A_{j}\left(x_{2}\right) \\
&+\left[A_{i}\left(x_{2}\right) F_{j}\left(x_{2}\right)+A_{j}\left(x_{2}\right) F_{i}\left(x_{2}\right)-\psi_{i}\left(x_{2}\right) \psi_{j}\left(x_{2}\right)\right] A_{r}\left(x_{1}\right) A_{s}\left(x_{1}\right) \\
&+\left[\psi_{s}\left(x_{1}\right) A_{r}\left(x_{1}\right)+\psi_{r}\left(x_{1}\right) A_{s}\left(x_{1}\right)\right]\left[\psi_{i}\left(x_{2}\right) A_{j}\left(x_{2}\right)+\psi_{j}\left(x_{2}\right) A_{i}\left(x_{2}\right)\right] \\
& \int d^{2} \theta d^{2} \bar{\theta} \Phi_{r}\left(x_{1}, \theta, \bar{\theta}\right) \Phi_{s}\left(x_{1}, \theta, \bar{\theta}\right) \bar{\Phi}_{i}\left(x_{2}, \theta, \bar{\theta}\right) \bar{\Phi}_{j}\left(x_{2}, \theta, \bar{\theta}\right)= \\
&=\quad-A_{i}^{\dagger}\left(x_{2}\right) A_{j}^{\dagger}\left(x_{2}\right) \square_{1} A_{r}\left(x_{1}\right) A_{s}\left(x_{1}\right) \\
&+ i\left[\overline{\psi_{i}}\left(x_{2}\right) A_{j}^{\dagger}\left(x_{2}\right)+\overline{\psi_{j}}\left(x_{2}\right) A_{i}^{\dagger}\left(x_{2}\right)\right] \bar{\sigma}^{\mu} \partial_{1, \mu}\left[\psi_{s}\left(x_{1}\right) A_{r}\left(x_{1}\right)+\psi_{r}\left(x_{1}\right) A_{s}\left(x_{1}\right)\right] \\
&+\left[A_{r}\left(x_{1}\right) F_{s}\left(x_{1}\right)+A_{s}\left(x_{1}\right) F_{r}\left(x_{1}\right)-\psi_{r}\left(x_{1}\right) \psi_{s}\left(x_{1}\right)\right] \times \\
& \times\left[A_{j}^{\dagger}\left(x_{2}\right) F_{i}^{\dagger}\left(x_{2}\right)+A_{i}^{\dagger}\left(x_{2}\right) F_{j}^{\dagger}\left(x_{2}\right)-\overline{\psi_{j}}\left(x_{2}\right) \overline{\psi_{i}}\left(x_{2}\right)\right]
\end{aligned}
$$

Here we have partially integrated derivatives and dropped total derivatives which do not contribute to the action. 


\section{Appendix $\mathrm{C}$}

\section{Feynman Rules}

In this appendix we present the component field Feynman rules that we have used in the calculations of chapters 1 and 23. Feynman rules for Majorana fermions which yield the correct relative minus signs between different diagrams contributing to a process without explicit recourse to Wick's theorem are proposed in ref. [52]. The basic idea is to introduce a continuous fermion flow, i.e. an arbitrary orientation of each fermion line. Then one can form chains of Dirac matrices by proceeding in a direction opposite to the chosen fermion flow. Relative signs of interfering diagrams are determined like for Dirac fermions, i.e. any permutation of two external fermion lines gives a minus sign. One only needs the Dirac propagator for all fermions. However, one has to introduce two analytical expressions for each vertex involving fermions, corresponding to the two different choices of the fermion flow.

As an example, consider the coupling of a right-handed neutrino to a SM Higgs and lepton doublet. This coupling can be written in two equivalent ways

$$
\bar{N} \lambda_{\nu}^{T} P_{\mathrm{L}}\left(l \epsilon H_{2}\right)=-\left(H_{2} \epsilon \overline{l^{c}}\right) P_{\mathrm{L}} \lambda_{\nu} \bar{N}
$$

corresponding to the two possible choices for the fermion flow. This gives rise to the following equivalent vertices, where the thin arrow denotes the chosen fermion flow
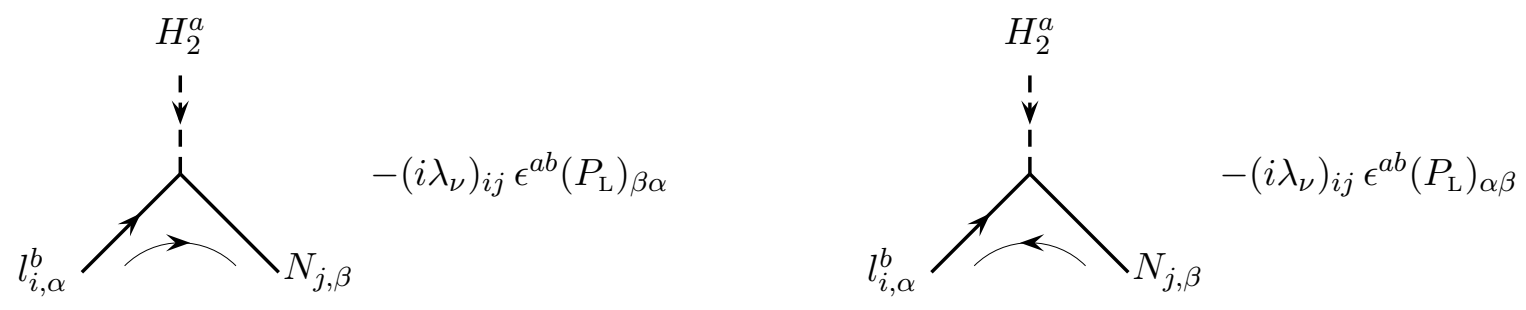
In general, one has spinor structures of the kind

$$
\overline{\psi_{1}} \Gamma \psi_{2}
$$

where the four-component spinors $\psi_{1}$ and $\psi_{2}$ are either Dirac or Majorana fermions, and $\Gamma$ is a product of Dirac $\gamma$-matrices,

$$
\Gamma=1, \gamma^{\mu}, \gamma^{5}, \gamma^{5} \gamma^{\mu}, \sigma^{\mu \nu}
$$

Reverting the fermion flow corresponds to replacing particles by antiparticles, i.e. (C.2) is rewritten in the equivalent form

$$
\overline{\psi_{1}} \Gamma \psi_{2}=\overline{\psi_{2}^{c}} \Gamma^{\prime} \psi_{1}^{c}
$$

where

$$
\Gamma^{\prime}=C \Gamma^{T} C^{-1}=\left\{\begin{array}{rl}
\Gamma & \text { for } \Gamma=1, \gamma^{5}, \gamma^{5} \gamma^{\mu} \\
-\Gamma & \text { for } \Gamma=\gamma^{\mu}, \sigma^{\mu \nu}
\end{array} .\right.
$$

Hence, when stating Feynman rules in the following we can restrict ourselves to one fermion flow. Changing the fermion flow just amounts to replacing $\Gamma$ by $\pm \Gamma$, according to eq. (C.5).

Decomposing the superfield products in the superpotential (3.1) into component fields, we get the Yukawa interactions of a right-handed Majorana neutrino
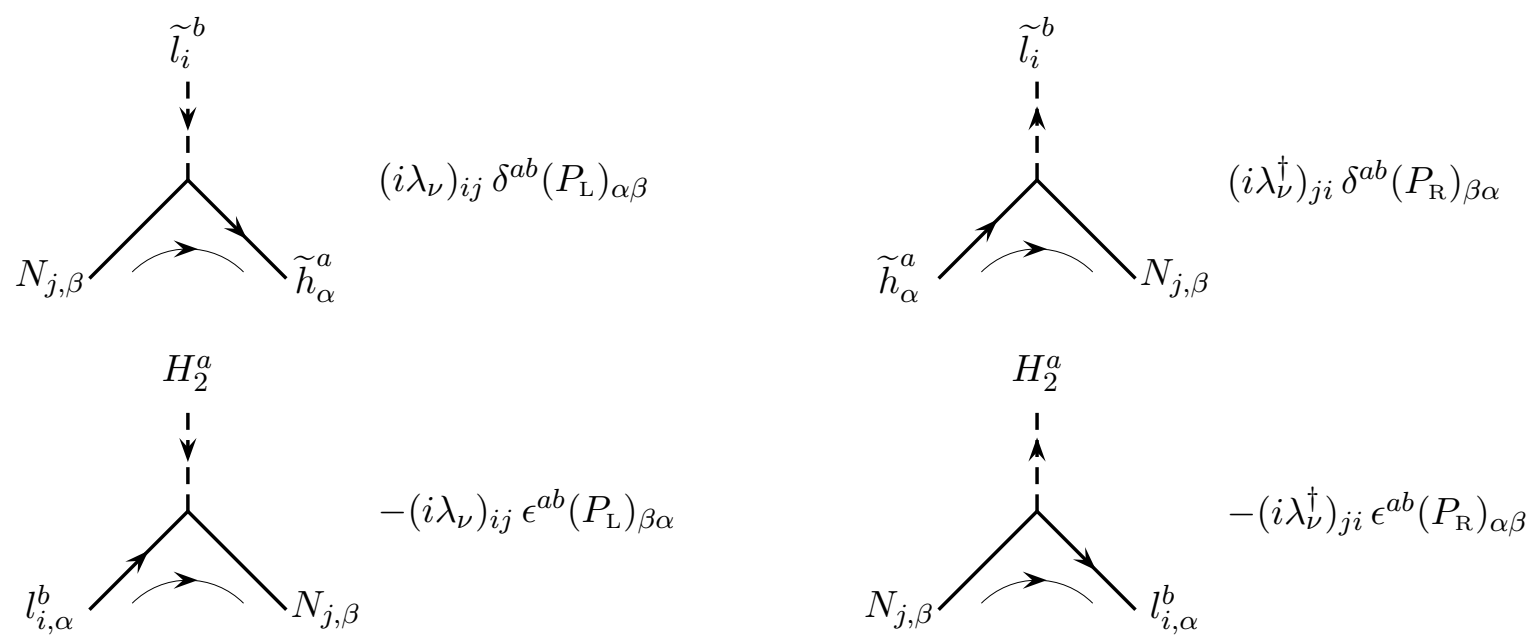

Correspondingly, the interactions of a scalar neutrino are given by
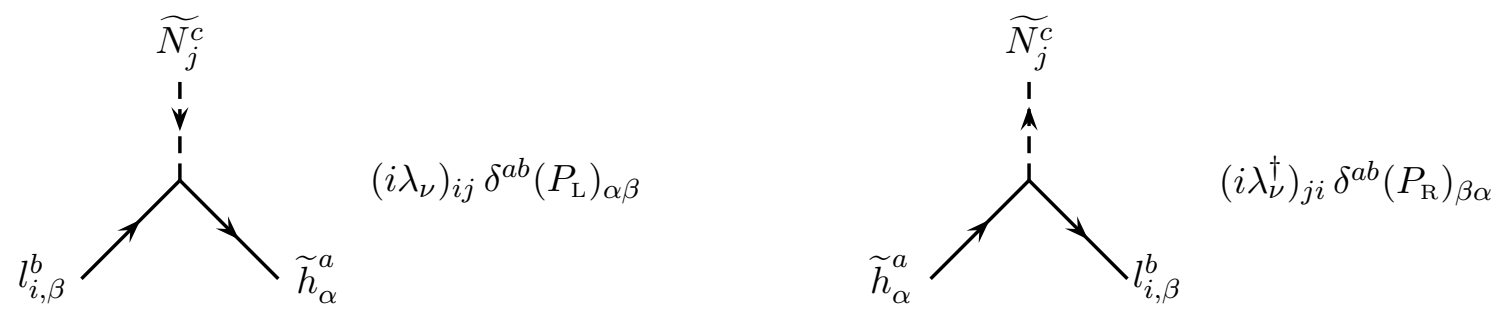
Here we have not specified an explicit fermion flow, since these diagrams have a natural orientation of the fermion lines.

The mass term in the auxiliary neutrino field $F_{N_{i}^{c}}$ (cf. eq. (3.22)) yields trilinear scalar couplings
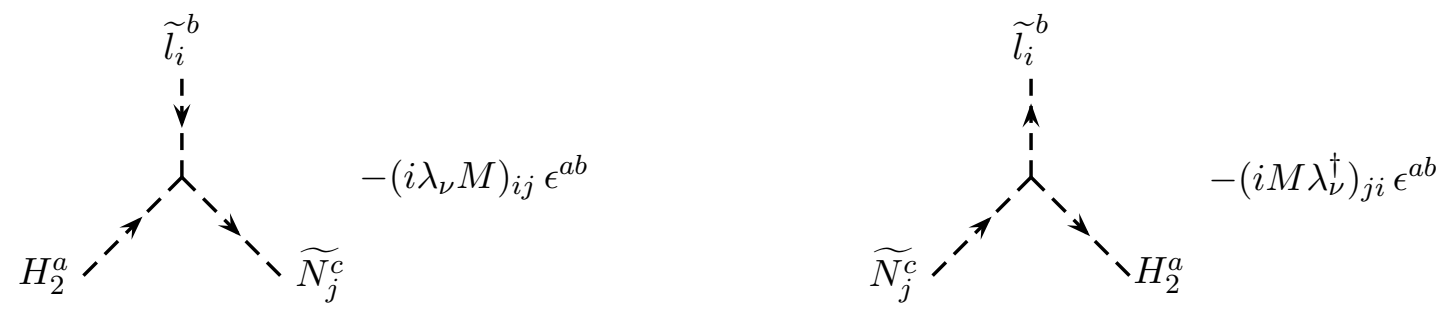

Furthermore, we take into account the following Yukawa couplings of the (s)top
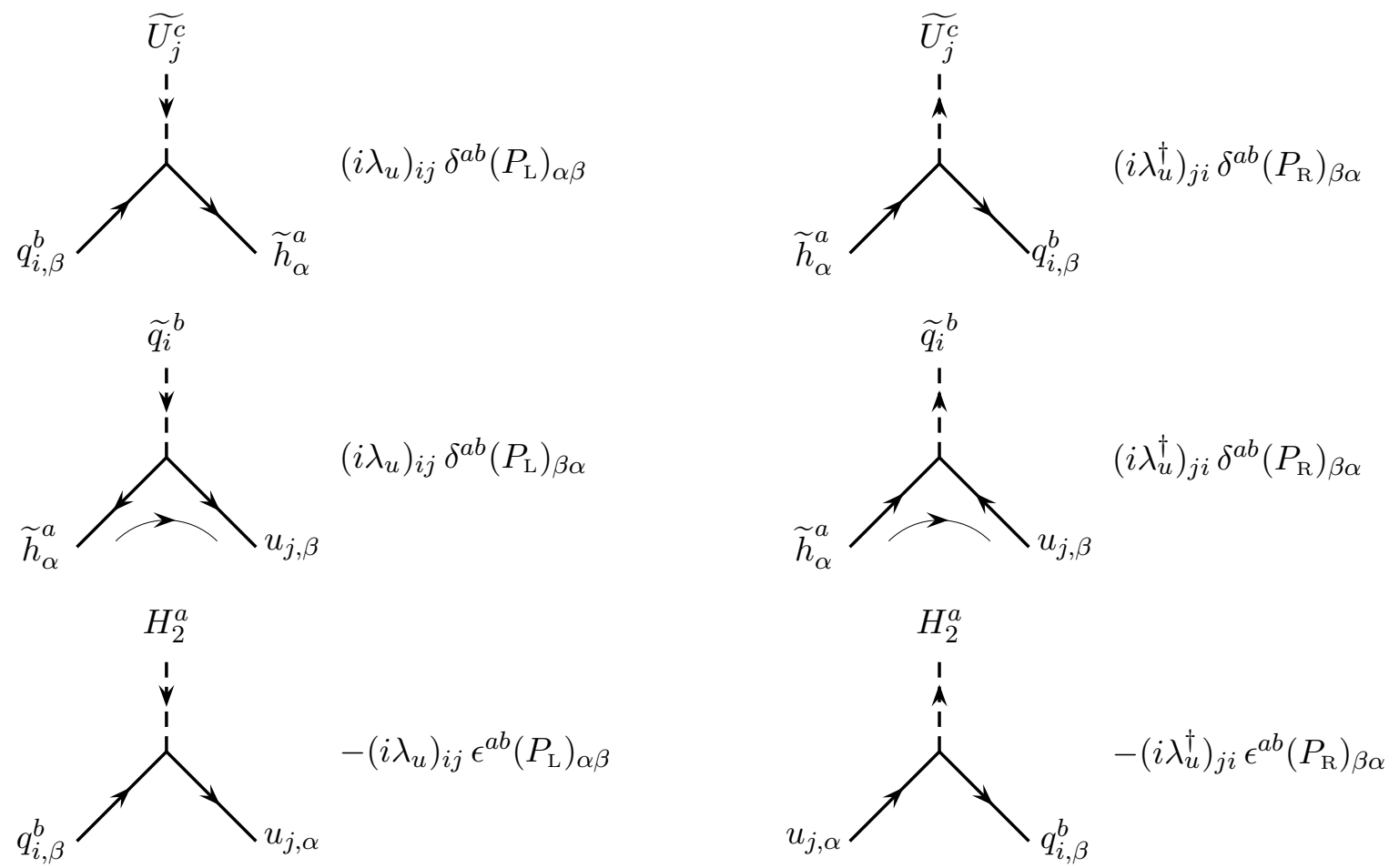

Finally, the scalar potential

$$
\mathcal{V}=\sum_{i} F_{i}^{\dagger} F_{i}, \quad i=H_{1}, H_{2}, Q_{i}, \ldots, N_{i}^{c}
$$

yields quartic scalar couplings involving one or two scalar neutrinos
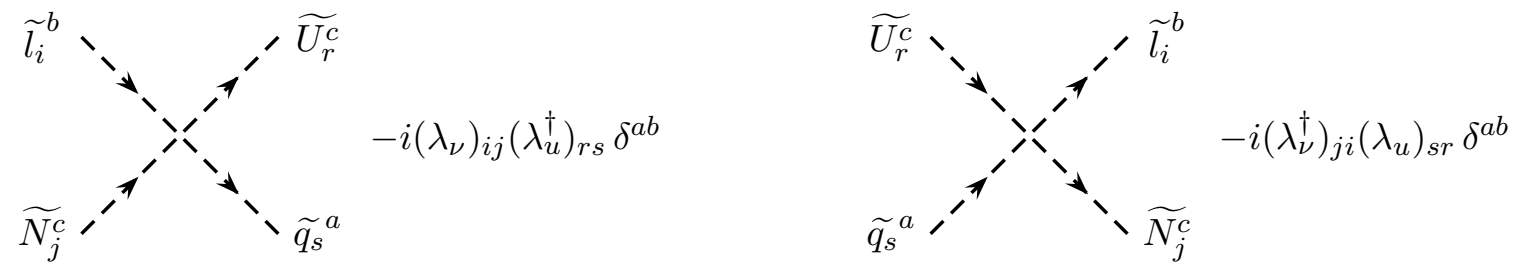


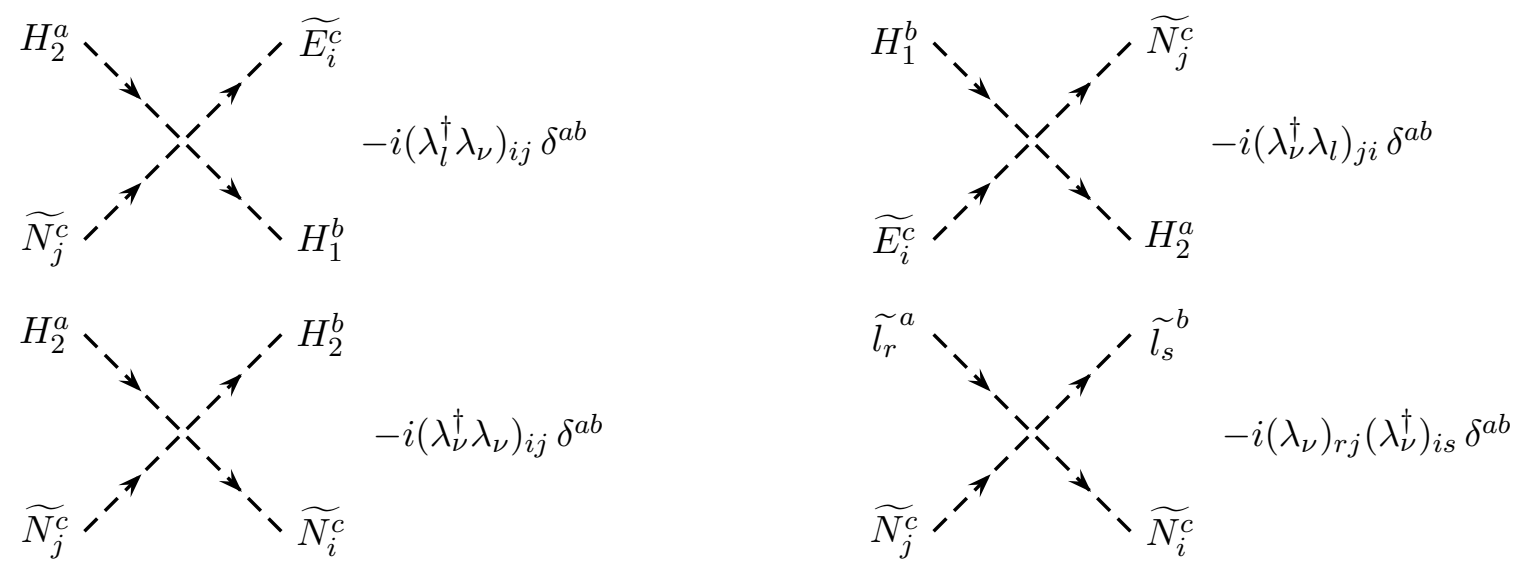

Internal Dirac or Majorana fermion lines are all represented by the usual Dirac propagator

$$
\alpha \stackrel{p}{\longrightarrow} \beta \quad\left(\frac{i}{\not p-m+i \epsilon}\right)_{\beta \alpha} .
$$

Correspondingly, we assign spinors to external fermion lines with orientation

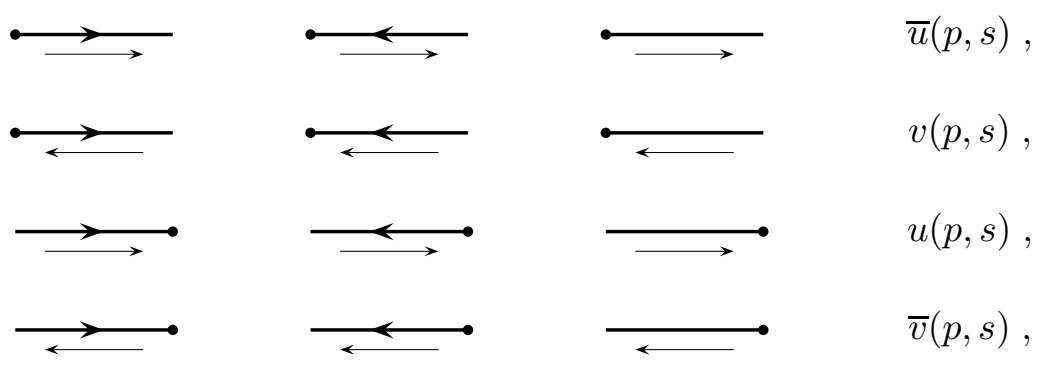

where the momentum $p$ always flows from left to right. The spinors $v$ and $u$ are related by charge conjugation

$$
\begin{aligned}
& v(p, s)=C \bar{u}^{T}(p, s) \quad \Leftrightarrow \quad \bar{u}(p, s)=v^{T}(p, s) C, \\
& u(p, s)=C \bar{v}^{T}(p, s) \quad \Leftrightarrow \quad \bar{v}(p, s)=u^{T}(p, s) C .
\end{aligned}
$$




\section{Appendix D}

\section{Kinetic Theory}

The microscopic evolution of particle densities and asymmetries is governed by a network of Boltzmann equations. In the following we will compile some basic formulae to introduce our notation [2].

\section{D.1 Thermodynamics in the Expanding Universe}

The early universe can be assumed to be spatially homogeneous and isotropic. Hence, it is described by a Robertson-Walker metric

$$
d s^{2}=d t^{2}-R(t)^{2}\left\{\frac{d r^{2}}{1-k r^{2}}+r^{2} d \theta^{2}+r^{2} \sin ^{2} \theta d \phi^{2}\right\}
$$

where $(t, r, \theta, \phi)$ are comoving coordinates and $k= \pm 1,0$ describes the spatial curvature of spacetime. The scale factor $R(t)$, which describes the expansion of the universe, is given by the Friedmann equation

$$
\dot{R}^{2}+k=\frac{8 \pi G}{3} \rho R^{2}
$$

where $\rho$ is the energy density of the universe, and

$$
G=\frac{1}{m_{P l}^{2}}
$$

denotes Newton's constant in units where $\hbar=c=1$, and $m_{P l}=1.2211 \cdot 10^{19} \mathrm{GeV}$ is the Planck mass. Neglecting the curvature term $k$ in the Friedmann equation (D.2), which is a good approximation in the early universe, we get an equation for the Hubble parameter $H$

$$
H \equiv \frac{\dot{R}}{R}=\frac{1}{m_{P l}} \sqrt{\frac{8 \pi \rho}{3}} .
$$


In a radiation dominated universe the energy density reads

$$
\rho=g_{*} \frac{\pi^{2}}{30} T^{4}
$$

where $g_{*}$ is the number of effectively massless degrees of freedom

$$
g_{*}=\sum_{i=\text { Bosons }} g_{i}+\frac{7}{8} \sum_{i=\text { Fermions }} g_{i}
$$

and $g_{i}$ is the number of internal degrees of freedom of the corresponding particle. At temperatures far above the electroweak scale one has $g_{*}=106.75$ in the standard model, and $g_{*}=228.75$ in the MSSM.

Hence, the Hubble parameter in a radiation dominated universe reads

$$
H=\sqrt{\frac{4 \pi^{3} g *}{45}} \frac{T^{2}}{m_{P l}} .
$$

\section{D.2 Boltzmann Equations}

It is usually a good approximation to assume Maxwell-Boltzmann statistics, so that the equilibrium number density of a particle $i$ is given by

$$
n_{i}^{\mathrm{eq}}(T)=\frac{g_{i}}{(2 \pi)^{3}} \int \mathrm{d}^{3} p_{i} f_{i}^{\mathrm{eq}} \quad \text { with } \quad f_{i}^{\mathrm{eq}}\left(E_{i}, T\right)=\mathrm{e}^{-E_{i} / T} .
$$

For a massive particle one finds

$$
n_{i}^{\mathrm{eq}}(T)=\frac{g_{i} T m_{i}^{2}}{2 \pi^{2}} \mathrm{~K}_{2}\left(\frac{m_{i}}{T}\right)
$$

whereas for a massless particle one gets

$$
n_{i}^{\mathrm{eq}}(T)=\frac{g_{i} T^{3}}{\pi^{2}} .
$$

Particle densities can be changed by interactions and by the expansion of the universe. Since we are only interested in the effect of interactions, it is useful to scale out the expansion. This is done by taking the number of particles per comoving volume element, i.e. the ratio of the particle density $n_{i}$ to the entropy density $s$,

$$
Y_{i}=\frac{n_{i}}{s},
$$

as independent variable instead of the number density. In a radiation dominated universe the entropy density reads

$$
s=g_{*} \frac{2 \pi^{2}}{45} T^{3} .
$$


In our case elastic scatterings, which can only change the phase space distributions but not the particle densities, occur at a much higher rate than inelastic processes. Therefore, we can assume kinetic equilibrium, so that the phase space densities are given by

$$
f_{i}\left(E_{i}, T\right)=\frac{n_{i}}{n_{i}^{\mathrm{eq}}} \mathrm{e}^{-E_{i} / T}
$$

In this framework the Boltzmann equation describing the evolution of a particle number $Y_{\psi}$ in an isentropically expanding universe reads 36,13 ,

$$
\begin{aligned}
\frac{\mathrm{d} Y_{\psi}}{\mathrm{d} z}=-\frac{z}{s H\left(m_{\psi}\right)} \sum_{a, i, j, \ldots} & {\left[\frac{Y_{\psi} Y_{a} \ldots}{Y_{\psi}^{\mathrm{eq}} Y_{a}^{\mathrm{eq}} \ldots} \gamma^{\mathrm{eq}}(\psi+a+\ldots \rightarrow i+j+\ldots)\right.} \\
& \left.-\frac{Y_{i} Y_{j} \ldots}{Y_{i}^{\mathrm{eq}} Y_{j}^{\mathrm{eq}} \ldots} \gamma^{\mathrm{eq}}(i+j+\ldots \rightarrow \psi+a+\ldots)\right],
\end{aligned}
$$

where $z=m_{\psi} / T$ and $H\left(m_{\psi}\right)$ is the Hubble parameter at $T=m_{\psi}$. The $\gamma^{\text {eq }}$ are space time densities of scatterings for the different processes. For a decay one finds 13]

$$
\gamma_{D}:=\gamma^{\mathrm{eq}}(\psi \rightarrow i+j+\ldots)=n_{\psi}^{\mathrm{eq}} \frac{\mathrm{K}_{1}(z)}{\mathrm{K}_{2}(z)} \Gamma
$$

where $K_{1}$ and $K_{2}$ are modified Bessel functions and $\Gamma$ is the usual decay width in the rest system of the decaying particle. Neglecting a possible $C P$ violation, one finds the same reaction density for the inverse decay.

The reaction density for a two body scattering reads

$$
\gamma^{\mathrm{eq}}(\psi+a \leftrightarrow i+j+\ldots)=\frac{T}{64 \pi^{4}} \int_{\left(m_{\psi}+m_{a}\right)^{2}}^{\infty} \mathrm{d} s \hat{\sigma}(s) \sqrt{s} \mathrm{~K}_{1}\left(\frac{\sqrt{s}}{T}\right)
$$

where $s$ is the squared centre of mass energy and the reduced cross section $\hat{\sigma}(s)$ for the process $\psi+a \rightarrow i+j+\ldots$ is related to the usual total cross section $\sigma(s)$ by

$$
\hat{\sigma}(s)=\frac{2 \lambda\left(s, m_{\psi}^{2}, m_{a}^{2}\right)}{s} \sigma(s),
$$

where $\lambda$ is the usual kinematical function

$$
\lambda\left(s, m_{\psi}^{2}, m_{a}^{2}\right) \equiv\left[s-\left(m_{\psi}+m_{a}\right)^{2}\right]\left[s-\left(m_{\psi}-m_{a}\right)^{2}\right] .
$$




\section{Appendix E}

\section{Reduced Cross Sections}

In this section we will collect the reduced cross sections for all the $2 \leftrightarrow 2$ and $2 \leftrightarrow 3$ processes that we had discussed in chapter 3. The corresponding reaction densities, which can be calculated analytically in some interesting limiting cases, will be discussed in the next appendix.

\section{E.1 Lepton Number Violating Processes Mediated by Right- Handed Neutrinos}

We have mentioned in the main text that we have to subtract the contributions coming from on-shell (s)neutrinos, i.e. we have to replace the usual propagators by off-shell propagators

$$
\frac{1}{D_{j}(x)}:=\frac{x-a_{j}}{\left(x-a_{j}\right)^{2}+a_{j} c_{j}} \quad \text { and } \quad \frac{1}{\widetilde{D}_{j}(x)}:=\frac{x-a_{j}}{\left(x-a_{j}\right)^{2}+a_{j} \widetilde{c_{j}}}
$$

To begin with, let us specify the reduced cross sections for the reactions depicted in fig. 3.5. For the processes $\widetilde{l}+\overline{\widetilde{h}} \leftrightarrow \widetilde{l}^{\dagger}+\widetilde{h}$ and $l+H_{2} \leftrightarrow \bar{l}+H_{2}^{\dagger}$ one has

$$
\begin{aligned}
& \hat{\sigma}_{N}^{(1)}(x)=\hat{\sigma}_{N}^{(2)}(x)=\frac{1}{2 \pi}\left\{\sum_{j}\left(\lambda_{\nu}^{\dagger} \lambda_{\nu}\right)_{j j}^{2} \frac{a_{j}}{x}\left[\frac{x}{a_{j}}+\frac{x}{D_{j}(x)}+\frac{x^{2}}{2 D_{j}^{2}(x)}-\left(1+\frac{x+a_{j}}{D_{j}(x)}\right) \ln \left(\frac{x+a_{j}}{a_{j}}\right)\right]\right. \\
& +\sum_{\substack{n, j \\
j<n}} \operatorname{Re}\left[\left(\lambda_{\nu}^{\dagger} \lambda_{\nu}\right)_{n j}^{2}\right] \frac{\sqrt{a_{n} a_{j}}}{x}\left[\frac{x}{D_{j}(x)}+\frac{x}{D_{n}(x)}+\frac{x^{2}}{D_{j}(x) D_{n}(x)}\right. \\
& \left.\left.\quad+\left(x+a_{j}\right)\left(\frac{2}{a_{n}-a_{j}}-\frac{1}{D_{n}(x)}\right) \ln \left(\frac{x+a_{j}}{a_{j}}\right)+\left(x+a_{n}\right)\left(\frac{2}{a_{j}-a_{n}}-\frac{1}{D_{j}(x)}\right) \ln \left(\frac{x+a_{n}}{a_{n}}\right)\right]\right\},
\end{aligned}
$$

where $n$ and $j$ are the flavour indices of the neutrinos in the intermediate state. The interference terms with $n \neq j$ are always very small and can safely be neglected. 
The reduced cross section for the process $\widetilde{l}+\overline{\widetilde{h}} \leftrightarrow \bar{l}+H_{2}^{\dagger}$ reads

$$
\begin{aligned}
\hat{\sigma}_{N}^{(3)}(x)= & \frac{1}{2 \pi}\left\{\sum_{j}\left(\lambda_{\nu}^{\dagger} \lambda_{\nu}\right)_{j j}^{2} \frac{a_{j}}{x}\left[\frac{-x}{x+a_{j}}+\frac{x}{D_{j}(x)}+\frac{x^{2}}{2 D_{j}^{2}(x)}+\left(1-\frac{a_{j}}{D_{j}(x)}\right) \ln \left(\frac{x+a_{j}}{a_{j}}\right)\right]\right. \\
& +\sum_{\substack{n, j \\
j<n}} \operatorname{Re}\left[\left(\lambda_{\nu}^{\dagger} \lambda_{\nu}\right)_{n j}^{2}\right] \frac{\sqrt{a_{n} a_{j}}}{x}\left[\frac{x}{D_{j}(x)}+\frac{x}{D_{n}(x)}+\frac{x^{2}}{D_{j}(x) D_{n}(x)}\right. \\
& \left.\left.-a_{j}\left(\frac{2}{a_{n}-a_{j}}+\frac{1}{D_{n}(x)}\right) \ln \left(\frac{x+a_{j}}{a_{j}}\right)-a_{n}\left(\frac{2}{a_{j}-a_{n}}+\frac{1}{D_{j}(x)}\right) \ln \left(\frac{x+a_{n}}{a_{n}}\right)\right]\right\} .
\end{aligned}
$$

The same result is valid for the $C P$ conjugated process.

For the process $l+\overline{\widetilde{h}} \leftrightarrow \widetilde{l}^{\dagger}+H_{2}^{\dagger}$ one finds

$$
\begin{gathered}
\hat{\sigma}_{N}^{(4)}(x)=\frac{1}{2 \pi}\left\{\sum_{j}\left(\lambda_{\nu}^{\dagger} \lambda_{\nu}\right)_{j j}^{2} \frac{a_{j}}{x}\left[\frac{x^{2}}{a_{j}\left(x+a_{j}\right)}+\frac{x^{2}}{\widetilde{D}_{j}^{2}(x)}+\frac{x}{\widetilde{D}_{j}(x)} \ln \left(\frac{x+a_{j}}{a_{j}}\right)\right]\right. \\
+\sum_{\substack{n, j \\
j<n}} \operatorname{Re}\left[\left(\lambda_{\nu}^{\dagger} \lambda_{\nu}\right)_{n j}^{2}\right] \frac{\sqrt{a_{n} a_{j}}}{x}\left[\frac{2 x^{2}}{\widetilde{D_{j}}(x) \widetilde{D_{n}}(x)}+x\left(\frac{2}{a_{n}-a_{j}}+\frac{1}{\widetilde{D_{n}(x)}}\right) \ln \left(\frac{x+a_{j}}{a_{j}}\right)\right. \\
\left.\left.+x\left(\frac{2}{a_{j}-a_{n}}+\frac{1}{\widetilde{D_{j}}(x)}\right) \ln \left(\frac{x+a_{n}}{a_{n}}\right)\right]\right\} .
\end{gathered}
$$

For the scattering $\widetilde{l}+H_{2} \rightarrow \widetilde{l}^{\dagger}+\widetilde{U^{c}}+\widetilde{q}$ and the corresponding $C P$ transformed process we have

$$
\begin{aligned}
\hat{\sigma}_{N}^{(5)}(x) & =\frac{3 \alpha_{u}}{8 \pi^{2}}\left\{\sum_{j}\left(\lambda_{\nu}^{\dagger} \lambda_{\nu}\right)_{j j}^{2} \frac{a_{j}}{x}\left[\frac{x}{a_{j}}+\frac{x}{\widetilde{D_{j}(x)}}+\frac{x^{2}}{\widetilde{D}_{j}^{2}(x)}-\left(1+\frac{x+a_{j}}{\widetilde{D_{j}}(x)}\right) \ln \left(\frac{x+a_{j}}{a_{j}}\right)\right]\right. \\
+ & \sum_{\substack{n, j \\
j<n}} \operatorname{Re}\left[\left(\lambda_{\nu}^{\dagger} \lambda_{\nu}\right)_{n j}^{2}\right] \frac{\sqrt{a_{n} a_{j}}}{x}\left[\frac{x}{\widetilde{D_{j}(x)}}+\frac{x}{\widetilde{D_{n}}(x)}+\frac{x^{2}}{\widetilde{D_{j}(x) \widetilde{D}_{n}(x)}}\right. \\
& \left.\left.+\left(x+a_{j}\right)\left(\frac{2}{a_{n}-a_{j}}-\frac{1}{\widetilde{D_{n}}(x)}\right) \ln \left(\frac{x+a_{j}}{a_{j}}\right)+\left(x+a_{n}\right)\left(\frac{2}{a_{j}-a_{n}}-\frac{1}{\widetilde{D_{j}}(x)}\right) \ln \left(\frac{x+a_{n}}{a_{n}}\right)\right]\right\} .
\end{aligned}
$$

Finally, we have two processes which do not violate lepton number but merely transform leptons into scalar leptons and vice versa. We have the $2 \rightarrow 2$ scattering $l+H_{2} \leftrightarrow \widetilde{l}+\overline{\widetilde{h}}$,

$$
\hat{\sigma}_{N}^{(6)}(x)=\frac{1}{4 \pi} \sum_{j, n}\left|\left(\lambda_{\nu}^{\dagger} \lambda_{\nu}\right)_{n j}\right|^{2} \frac{x^{2}}{D_{j}(x) D_{n}(x)},
$$

and the $2 \rightarrow 3$ process $l+\overline{\widetilde{h}} \leftrightarrow \widetilde{l}+\widetilde{q}^{\dagger}+\widetilde{U}^{\dagger}$,

$$
\hat{\sigma}_{N}^{(7)}(x)=\frac{3 \alpha_{u}}{16 \pi^{2}} \sum_{j, n}\left|\left(\lambda_{\nu}^{\dagger} \lambda_{\nu}\right)_{n j}\right|^{2} \frac{x^{2}}{\widetilde{D_{j}}(x) \widetilde{D_{n}}(x)} .
$$


Let us now come to the $2 \rightarrow 3$ processes shown in fig. 3.6.

For the transition $\widetilde{q}+\widetilde{U^{c}} \rightarrow \widetilde{l}+\widetilde{l}+H_{2}$ the reduced cross section reads

$$
\begin{aligned}
& \hat{\sigma}_{N}^{(8)}(x)=\frac{3 \alpha_{u}}{16 \pi^{2}}\left\{\sum _ { j } ( \lambda _ { \nu } ^ { \dagger } \lambda _ { \nu } ) _ { j j } ^ { 2 } \frac { a _ { j } } { x } \left[-\frac{x}{a_{j}+\widetilde{c_{j}}}+\frac{x-a_{j}}{\sqrt{a_{j} \widetilde{c_{j}}}}\left[\arctan \left(\frac{x-a_{j}}{\sqrt{a_{j} \widetilde{c_{j}}}}\right)+\arctan \left(\sqrt{\frac{a_{j}}{\widetilde{c_{j}}}}\right)\right]\right.\right. \\
& \left.\quad-\ln \left(\frac{\left(x-a_{j}\right)^{2}+a_{j} \widetilde{c_{j}}}{a_{j}^{2}+a_{j} \widetilde{c_{j}}}\right)+\frac{1}{2} \int_{0}^{x} \mathrm{~d} x_{1} \frac{1}{\widetilde{D_{j}}\left(x_{1}\right)} \ln \left(\frac{\left(x-x_{1}-a_{j}\right)^{2}+a_{j} \widetilde{c_{j}}}{a_{j}^{2}+a_{j} \widetilde{c_{j}}}\right)\right] \\
& +2 \sum_{\substack{n, j \\
j<n}} \operatorname{Re}\left[\left(\lambda_{\nu}^{\dagger} \lambda_{\nu}\right)_{n j}^{2}\right] \frac{\sqrt{a_{n} a_{j}}}{x}\left[\frac{1}{2} \int_{0}^{x} \mathrm{~d} x_{1} \frac{1}{\widetilde{D_{n}}\left(x_{1}\right)} \ln \left(\frac{\left(x-x_{1}-a_{j}\right)^{2}+a_{j} \widetilde{c_{j}}}{a_{j}^{2}+a_{j} \widetilde{c_{j}}}\right)\right. \\
& +2 \sqrt{\widetilde{a_{j} \widetilde{c_{j}}}} \frac{x-a_{n}}{\left(a_{j}-a_{n}\right)^{2}}\left[\arctan \left(\frac{x-a_{j}}{\sqrt{a_{j} \widetilde{c_{j}}}}\right)+\arctan \left(\sqrt{\frac{a_{j}}{\widetilde{c_{j}}}}\right)\right]+\frac{x-a_{j}}{a_{j}-a_{n}} \ln \left(\frac{\left(x-a_{j}\right)^{2}+a_{j} \widetilde{c_{j}}}{a_{j}^{2}+a_{j} \widetilde{c_{j}}}\right) \\
& \left.\left.\quad+2 \sqrt{a_{n} \widetilde{c_{n}}} \frac{x-a_{j}}{\left(a_{n}-a_{j}\right)^{2}}\left[\arctan \left(\frac{x-a_{n}}{\sqrt{a_{n} \widetilde{c_{n}}}}\right)+\arctan \left(\sqrt{\frac{a_{n}}{\widetilde{c_{n}}}}\right)\right]+\frac{x-a_{n}}{a_{n}-a_{j}} \ln \left(\frac{\left(x-a_{n}\right)^{2}+a_{n} \widetilde{c_{n}}}{a_{n}^{2}+a_{n} \widetilde{c_{n}}}\right)\right]\right\} .
\end{aligned}
$$

The remaining integral cannot be solved exactly. However, it can be neglected for $x>a_{j}, a_{n}$ and for $x<a_{j}, a_{n}$ it can be approximated by

$$
\begin{aligned}
& \frac{1}{2} \int_{0}^{x} \mathrm{~d} x_{1} \frac{1}{\widetilde{D_{n}}\left(x_{1}\right)} \ln \left(\frac{\left(x-x_{1}-a_{j}\right)^{2}+a_{j} \widetilde{c_{j}}}{a_{j}^{2}+a_{j} \widetilde{c_{j}}}\right) \\
& \quad \approx \ln \left(\frac{a_{j}+a_{n}-x}{a_{j}}\right) \ln \left(\frac{a_{n}-x}{a_{n}}\right)+\operatorname{Sp}\left(\frac{a_{n}}{a_{n}+a_{j}-x}\right)-\operatorname{Sp}\left(\frac{a_{n}-x}{a_{n}+a_{j}-x}\right),
\end{aligned}
$$

where $\operatorname{Sp}(x)$ is the Spence function or dilogarithm

$$
\operatorname{Sp}(x)=\operatorname{Li}_{2}(x)=-\int_{0}^{x} d y \frac{\ln (1-y)}{y}
$$

For the scatterings $\widetilde{q}+\widetilde{U^{c}} \rightarrow \widetilde{l}+\bar{l}+\widetilde{h}, \widetilde{l}^{\dagger}+\widetilde{q} \rightarrow \bar{l}+{\widetilde{U^{c}}}^{\dagger}+\widetilde{h}$ and $\widetilde{l}^{\dagger}+\widetilde{U^{c}} \rightarrow \bar{l}+\widetilde{q}^{\dagger}+\widetilde{h}$ the reduced cross sections are equal,

$$
\begin{aligned}
\hat{\sigma}_{N}^{(9)}(x)=\hat{\sigma}_{N}^{(11)}(x) & =\frac{3 \alpha_{u}}{8 \pi^{2} x}\left\{\sum _ { j } ( \lambda _ { \nu } ^ { \dagger } \lambda _ { \nu } ) _ { j j } ^ { 2 } \left[-\frac{3}{2} x+\frac{1}{2}\left(x-2 a_{j}\right) \ln \left(\frac{\left(x-a_{j}\right)^{2}+a_{j} \widetilde{c_{j}}}{a_{j}^{2}+a_{j} \widetilde{c_{j}}}\right)\right.\right. \\
+ & \left.\frac{1}{2} \sqrt{\frac{a_{j}}{\widetilde{c_{j}}}}\left(x-a_{j}+3 \widetilde{c_{j}}\right)\left[\arctan \left(\frac{x-a_{j}}{\sqrt{a_{j} \widetilde{c_{j}}}}\right)+\arctan \left(\sqrt{\frac{a_{j}}{\widetilde{c_{j}}}}\right)\right]\right]
\end{aligned}
$$




$$
\begin{aligned}
+2 \sum_{\substack{n, j \\
j<n}}\left|\left(\lambda_{\nu}^{\dagger} \lambda_{\nu}\right)_{n j}\right|^{2}\left[-2 x+a_{j} \frac{x-a_{j}}{a_{j}-a_{n}} \ln \left(\frac{\left(x-a_{j}\right)^{2}+a_{j} \widetilde{c_{j}}}{a_{j}^{2}+a_{j} \widetilde{c_{j}}}\right)+a_{n} \frac{x-a_{n}}{a_{n}-a_{j}} \ln \left(\frac{\left(x-a_{n}\right)^{2}+a_{n} \widetilde{c_{n}}}{a_{n}^{2}+a_{n} \widetilde{c_{n}}}\right)\right. \\
+2 \sqrt{a_{j} \widetilde{c_{j}}} \frac{x a_{n}-2 a_{n} a_{j}+a_{j}^{2}}{\left(a_{j}-a_{n}\right)^{2}}\left[\arctan \left(\frac{x-a_{j}}{\sqrt{a_{j}}}\right)+\arctan \left(\sqrt{\frac{a_{j}}{\widetilde{c_{j}}}}\right)\right] \\
\left.\left.+2 \sqrt{a_{n} \widetilde{c_{n}}} \frac{x a_{j}-2 a_{n} a_{j}+a_{n}^{2}}{\left(a_{n}-a_{j}\right)^{2}}\left[\arctan \left(\frac{x-a_{n}}{\sqrt{a_{n} \widetilde{c_{n}}}}\right)+\arctan \left(\sqrt{\frac{a_{n}}{\widetilde{c_{n}}}}\right)\right]\right]\right\} .
\end{aligned}
$$

For the process $\widetilde{l}^{\dagger}+\widetilde{q} \rightarrow \widetilde{l}+{\widetilde{U^{c}}}^{\dagger}+H_{2}$ and similar reactions one gets

$$
\begin{aligned}
& \hat{\sigma}_{N}^{(10)}(x)=\frac{3 \alpha_{u}}{16 \pi^{2}}\left\{\sum _ { j } ( \lambda _ { \nu } ^ { \dagger } \lambda _ { \nu } ) _ { j j } ^ { 2 } \frac { a _ { j } } { x } \left[\frac{x}{a_{j}}-2 \ln \left(\frac{x+a_{j}}{a_{j}}\right)-\ln \left(\frac{\left(x-a_{j}\right)^{2}+a_{j} \widetilde{c_{j}}}{a_{j}^{2}+a_{j} \widetilde{c_{j}}}\right)\right.\right. \\
& \left.+\frac{x-a_{j}}{\sqrt{a_{j} \widetilde{c_{j}}}}\left[\arctan \left(\frac{x-a_{j}}{\sqrt{a_{j} \widetilde{c}_{j}}}\right)+\arctan \left(\sqrt{\frac{a_{j}}{\widetilde{c_{j}}}}\right)\right]+2 \int_{0}^{x} \mathrm{~d} x_{1} \frac{1}{\widetilde{D_{j}}\left(x_{1}\right)}\left[\operatorname{Sp}\left(-\frac{x}{a_{j}}\right)-\operatorname{Sp}\left(-\frac{x_{1}}{a_{j}}\right)\right]\right] \\
& +2 \sum_{\substack{n, j \\
j<n}} \operatorname{Re}\left[\left(\lambda_{\nu}^{\dagger} \lambda_{\nu}\right)_{n j}^{2}\right] \frac{\sqrt{a_{n} a_{j}}}{x}\left[2 \frac{x+a_{j}}{a_{n}-a_{j}} \ln \left(\frac{x+a_{j}}{a_{j}}\right)+2 \frac{x+a_{n}}{a_{j}-a_{n}} \ln \left(\frac{x+a_{n}}{a_{n}}\right)\right. \\
& +\frac{x-a_{j}}{a_{j}-a_{n}} \ln \left(\frac{\left(x-a_{j}\right)^{2}+a_{j} \widetilde{c_{j}}}{a_{j}^{2}+a_{j} \widetilde{c_{j}}}\right)+2 \sqrt{a_{j} \widetilde{c_{j}}} \frac{x-a_{n}}{\left(a_{j}-a_{n}\right)^{2}}\left[\arctan \left(\frac{x-a_{j}}{\sqrt{a_{j} \widetilde{c_{j}}}}\right)+\arctan \left(\sqrt{\frac{a_{j}}{\widetilde{c_{j}}}}\right)\right] \\
& +\frac{x-a_{n}}{a_{n}-a_{j}} \ln \left(\frac{\left(x-a_{n}\right)^{2}+a_{n} \widetilde{c_{n}}}{a_{n}^{2}+a_{n} \widetilde{c_{n}}}\right)+2 \sqrt{a_{n} \widetilde{c_{n}}} \frac{x-a_{j}}{\left(a_{n}-a_{j}\right)^{2}}\left[\arctan \left(\frac{x-a_{n}}{\sqrt{a_{n} \widetilde{c_{n}}}}\right)+\arctan \left(\sqrt{\frac{a_{n}}{\widetilde{c_{n}}}}\right)\right] \\
& \left.+\int_{0}^{x} \mathrm{~d} x_{1}\left[\frac{1}{\widetilde{D_{j}}\left(x_{1}\right)}\left(\operatorname{Sp}\left(-\frac{x}{a_{n}}\right)-\operatorname{Sp}\left(-\frac{x_{1}}{a_{n}}\right)\right)+\frac{1}{\widetilde{D_{n}\left(x_{1}\right)}}\left(\operatorname{Sp}\left(-\frac{x}{a_{j}}\right)-\operatorname{Sp}\left(-\frac{x_{1}}{a_{j}}\right)\right)\right]\right] .
\end{aligned}
$$

The remaining integral can again not be solved exactly. However, it can be approximated by

$$
\begin{aligned}
& \int_{0}^{x} \mathrm{~d} x_{1} \frac{1}{\widetilde{D_{j}}\left(x_{1}\right)}\left[\operatorname{Sp}\left(-\frac{x}{a_{n}}\right)-\operatorname{Sp}\left(-\frac{x_{1}}{a_{n}}\right)\right] \\
& \quad \approx \frac{x}{a_{n}}-\frac{\sqrt{a_{j} \widetilde{c_{j}}}}{a_{n}}\left[\arctan \left(\frac{x-a_{j}}{\sqrt{a_{j} \widetilde{c_{j}}}}\right)+\arctan \left(\sqrt{\frac{a_{j}}{\widetilde{c_{j}}}}\right)\right]-\frac{x-a_{j}}{2 a_{n}} \ln \left(\frac{\left(x-a_{j}\right)^{2}+a_{j} \widetilde{c_{j}}}{a_{j}^{2}+a_{j} \widetilde{c_{j}}}\right)
\end{aligned}
$$

for $x<a_{n}$ and for $x>a_{n}$ it can be neglected.

Finally, we have to compute the $t$ - and $u$-channel processes in fig. 3.7, which give simple contributions. 
For the processes $\widetilde{l}+\widetilde{l} \leftrightarrow \widetilde{h}+\widetilde{h}$ and $l+l \leftrightarrow H_{2}^{\dagger}+H_{2}^{\dagger}$ we get

$$
\begin{aligned}
& \hat{\sigma}_{N}^{(12)}(x)=\hat{\sigma}_{N}^{(13)}(x)= \frac{1}{2 \pi}\left\{\sum_{j}\left(\lambda_{\nu}^{\dagger} \lambda_{\nu}\right)_{j j}^{2}\left[\frac{x}{x+a_{j}}+\frac{a_{j}}{x+2 a_{j}} \ln \left(\frac{x+a_{j}}{a_{j}}\right)\right]\right. \\
&+\sum_{\substack{n, j \\
j<n}} \operatorname{Re}\left[\left(\lambda_{\nu}^{\dagger} \lambda_{\nu}\right)_{n j}^{2}\right] \sqrt{a_{n} a_{j}}\left[\left(\frac{1}{x+a_{n}+a_{j}}+\frac{2}{a_{n}-a_{j}}\right) \ln \left(\frac{x+a_{j}}{a_{j}}\right)\right. \\
&\left.\left.+\left(\frac{1}{x+a_{n}+a_{j}}+\frac{2}{a_{j}-a_{n}}\right) \ln \left(\frac{x+a_{n}}{a_{n}}\right)\right]\right\} .
\end{aligned}
$$

In this order of perturbation theory the same result is valid for the $C P$ transformed processes.

For the scattering $\widetilde{l}+l \leftrightarrow \widetilde{h}+H_{2}^{\dagger}$ one has

$$
\begin{gathered}
\hat{\sigma}_{N}^{(14)}(x)=\frac{1}{2 \pi}\left\{\sum_{j}\left(\lambda_{\nu}^{\dagger} \lambda_{\nu}\right)_{j j}^{2}\left[\frac{x}{x+a_{j}}-\frac{a_{j}}{x+2 a_{j}} \ln \left(\frac{x+a_{j}}{a_{j}}\right)\right]\right. \\
+2 \sum_{\substack{n, j \\
j<n}} \operatorname{Re}\left[\left(\lambda_{\nu}^{\dagger} \lambda_{\nu}\right)_{n j}^{2}\right] \sqrt{a_{n} a_{j}}\left[\left(\frac{1}{x+a_{n}+a_{j}}+\frac{1}{a_{n}-a_{j}}\right) \ln \left(\frac{x+a_{j}}{a_{j}}\right)\right. \\
\left.\left.+\left(\frac{1}{x+a_{n}+a_{j}}+\frac{1}{a_{j}-a_{n}}\right) \ln \left(\frac{x+a_{n}}{a_{n}}\right)\right]\right\} .
\end{gathered}
$$

The $2 \rightarrow 3$ process $H_{2}+\widetilde{q}^{\dagger} \leftrightarrow \widetilde{l}^{\dagger}+\widetilde{l}^{\dagger}+\widetilde{U^{c}}$ gives

$$
\begin{aligned}
& \hat{\sigma}_{N}^{(15)}(x)=\frac{3 \alpha_{u}}{8 \pi^{2}}\left\{\sum _ { j } ( \lambda _ { \nu } ^ { \dagger } \lambda _ { \nu } ) _ { j j } ^ { 2 } \frac { a _ { j } } { x } \left[\frac{x}{a_{j}}-\left(1-\frac{1}{2} \ln \left(\frac{x+2 a_{j}}{a_{j}}\right)\right) \ln \left(\frac{x+a_{j}}{a_{j}}\right)\right.\right. \\
& \left.\quad+\frac{1}{2} \operatorname{Sp}\left(\frac{a_{j}}{x+2 a_{j}}\right)-\frac{1}{2} \operatorname{Sp}\left(\frac{x+a_{j}}{x+2 a_{j}}\right)\right] \\
& +\sum_{\substack{n, j \\
j<n}} \operatorname{Re}\left[\left(\lambda_{\nu}^{\dagger} \lambda_{\nu}\right)_{n j}^{2}\right] \frac{\sqrt{a_{n} a_{j}}}{x}\left[\left(2 \frac{x+a_{j}}{a_{n}-a_{j}}+\ln \left(\frac{x+a_{n}+a_{j}}{a_{n}}\right)\right) \ln \left(\frac{x+a_{j}}{a_{j}}\right)\right. \\
& +\left(2 \frac{x+a_{n}}{a_{j}-a_{n}}+\ln \left(\frac{x+a_{n}+a_{j}}{a_{j}}\right)\right) \ln \left(\frac{x+a_{n}}{a_{n}}\right) \\
& \left.\left.+\operatorname{Sp}\left(\frac{a j}{x+a_{n}+a_{j}}\right)-\operatorname{Sp}\left(\frac{x+a j}{x+a_{n}+a_{j}}\right)+\operatorname{Sp}\left(\frac{a n}{x+a_{n}+a_{j}}\right)-\operatorname{Sp}\left(\frac{x+a n}{x+a_{n}+a_{j}}\right)\right]\right\} .
\end{aligned}
$$

For the related transition $\widetilde{l}+\widetilde{l} \leftrightarrow \widetilde{U^{c}}+\widetilde{q}+H_{2}^{\dagger}$ we have

$$
\hat{\sigma}_{N}^{(16)}(x)=\frac{3 \alpha_{u}}{16 \pi^{2}}\left\{\sum _ { j } ( \lambda _ { \nu } ^ { \dagger } \lambda _ { \nu } ) _ { j j } ^ { 2 } \frac { a _ { j } } { x } \left[\frac{x}{a_{j}}+2 \operatorname{Sp}\left(-\frac{x+a_{j}}{a_{j}}\right)+\frac{\pi^{2}}{6}\right.\right.
$$




$$
\begin{gathered}
\left.-\left(1-2 \ln \left(\frac{x+2 a_{j}}{a_{j}}\right)\right) \ln \left(\frac{x+a_{j}}{a_{j}}\right)\right] \\
+2 \sum_{\substack{n, j \\
j<n}} \operatorname{Re}\left[\left(\lambda_{\nu}^{\dagger} \lambda_{\nu}\right)_{n j}^{2}\right] \frac{\sqrt{a_{n} a_{j}}}{x}\left[\left(\frac{x+a_{j}}{a_{n}-a_{j}}+\ln \left(\frac{x+a_{n}+a_{j}}{a_{n}}\right)\right) \ln \left(\frac{x+a_{j}}{a_{j}}\right)+\operatorname{Sp}\left(-\frac{x+a_{j}}{a_{n}}\right)\right. \\
\left.\left.+\left(\frac{x+a_{n}}{a_{j}-a_{n}}+\ln \left(\frac{x+a_{n}+a_{j}}{a_{j}}\right)\right) \ln \left(\frac{x+a_{n}}{a_{n}}\right)+\operatorname{Sp}\left(-\frac{x+a_{n}}{a_{j}}\right)+\frac{\pi^{2}}{6}+\frac{1}{2} \ln ^{2}\left(\frac{a_{n}}{a_{j}}\right)\right]\right\} .
\end{gathered}
$$

There are some $2 \rightarrow 2$ processes left which do not violate lepton number but simply transform leptons into scalar leptons, like in the process $\widetilde{l}+\bar{l} \leftrightarrow \widetilde{h}+H_{2}$

$$
\begin{aligned}
\hat{\sigma}_{N}^{(17)}(x) & =\frac{1}{2 \pi}\left\{\sum_{j}\left(\lambda_{\nu}^{\dagger} \lambda_{\nu}\right)_{j j}^{2}\left[\frac{-x}{x+a_{j}}+\ln \left(\frac{x+a_{j}}{a_{j}}\right)\right]\right. \\
& \left.+2 \sum_{\substack{n, j \\
j<n}}\left|\left(\lambda_{\nu}^{\dagger} \lambda_{\nu}\right)_{n j}\right|^{2}\left[\frac{a_{j}}{a_{j}-a_{n}} \ln \left(\frac{x+a_{j}}{a_{j}}\right)+\frac{a_{n}}{a_{n}-a_{j}} \ln \left(\frac{x+a_{n}}{a_{n}}\right)\right]\right\},
\end{aligned}
$$

or in the similar process $\widetilde{l}+H_{2}^{\dagger} \leftrightarrow \widetilde{h}+l$

$$
\begin{aligned}
\hat{\sigma}_{N}^{(18)}(x) & =\frac{1}{2 \pi}\left\{\sum_{j}\left(\lambda_{\nu}^{\dagger} \lambda_{\nu}\right)_{j j}^{2}\left[-2+\frac{x+2 a_{j}}{x} \ln \left(\frac{x+a_{j}}{a_{j}}\right)\right]\right. \\
& \left.+2 \sum_{\substack{n, j \\
j<n}}\left|\left(\lambda_{\nu}^{\dagger} \lambda_{\nu}\right)_{n j}\right|^{2}\left[-1+\frac{a_{j}}{x} \frac{x+a_{j}}{a_{j}-a_{n}} \ln \left(\frac{x+a_{j}}{a_{j}}\right)+\frac{a_{n}}{x} \frac{x+a_{n}}{a_{n}-a_{j}} \ln \left(\frac{x+a_{n}}{a_{n}}\right)\right]\right\} .
\end{aligned}
$$

Finally, the last process $l+\widetilde{l}^{\dagger} \leftrightarrow \widetilde{h}+\widetilde{q}^{\dagger}+{\widetilde{U^{c}}}^{\dagger}$ gives

$$
\begin{aligned}
\hat{\sigma}_{N}^{(19)}(x) & =\frac{3 \alpha_{u}}{8 \pi^{2}}\left\{\sum_{j}\left(\lambda_{\nu}^{\dagger} \lambda_{\nu}\right)_{j j}^{2}\left[-2+\frac{x+2 a_{j}}{x} \ln \left(\frac{x+a_{j}}{a_{j}}\right)\right]\right. \\
& \left.+2 \sum_{\substack{n, j \\
j<n}}\left|\left(\lambda_{\nu}^{\dagger} \lambda_{\nu}\right)_{n j}\right|^{2}\left[-1+\frac{a_{j}}{x} \frac{x+a_{j}}{a_{j}-a_{n}} \ln \left(\frac{x+a_{j}}{a_{j}}\right)+\frac{a_{n}}{x} \frac{x+a_{n}}{a_{n}-a_{j}} \ln \left(\frac{x+a_{n}}{a_{n}}\right)\right]\right\} .
\end{aligned}
$$

\section{E.2 Scattering off a Top or a Stop}

For the processes specified in fig. 3.9 the reduced cross sections read

$$
\hat{\sigma}_{t_{j}}^{(0)}=\frac{3 \alpha_{u}}{2}\left(\lambda_{\nu}^{\dagger} \lambda_{\nu}\right)_{j j} \frac{x^{2}-a_{j}^{2}}{x^{2}},
$$




$$
\begin{aligned}
& \hat{\sigma}_{t_{j}}^{(1)}=3 \alpha_{u}\left(\lambda_{\nu}^{\dagger} \lambda_{\nu}\right)_{j j} \frac{x-a_{j}}{x}\left[-\frac{2 x-a_{j}+2 a_{h}}{x-a_{j}+a_{h}}+\frac{x+2 a_{h}}{x-a_{j}} \ln \left(\frac{x-a_{j}+a_{h}}{a_{h}}\right)\right], \\
& \hat{\sigma}_{t_{j}}^{(2)}=3 \alpha_{u}\left(\lambda_{\nu}^{\dagger} \lambda_{\nu}\right)_{j j} \frac{x-a_{j}}{x}\left[-\frac{x-a_{j}}{x-a_{j}+2 a_{h}}+\ln \left(\frac{x-a_{j}+a_{h}}{a_{h}}\right)\right], \\
& \hat{\sigma}_{t_{j}}^{(3)}=3 \alpha_{u}\left(\lambda_{\nu}^{\dagger} \lambda_{\nu}\right)_{j j}\left(\frac{x-a_{j}}{x}\right)^{2}, \\
& \hat{\sigma}_{t_{j}}^{(4)}=3 \alpha_{u}\left(\lambda_{\nu}^{\dagger} \lambda_{\nu}\right)_{j j} \frac{x-a_{j}}{x}\left[\frac{x-2 a_{j}+2 a_{h}}{x-a_{j}+a_{h}}+\frac{a_{j}-2 a_{h}}{x-a_{j}} \ln \left(\frac{x-a_{j}+a_{h}}{a_{h}}\right)\right] .
\end{aligned}
$$

To regularize an infrared divergence in the $t$-channel diagrams we had to introduce a Higgs-mass

$$
a_{h}:=\left(\frac{\mu}{M_{1}}\right)^{2}
$$

In the calculations we have used the value $\mu=800 \mathrm{GeV}$.

The analogous processes involving a scalar neutrino (cf. fig. 3.10) give similar contributions

$$
\begin{aligned}
\hat{\sigma}_{t_{j}}^{(5)} & =\frac{3 \alpha_{u}}{2}\left(\lambda_{\nu}^{\dagger} \lambda_{\nu}\right)_{j j}\left(\frac{x-a_{j}}{x}\right)^{2}, \\
\hat{\sigma}_{t_{j}}^{(6)} & =3 \alpha_{u}\left(\lambda_{\nu}^{\dagger} \lambda_{\nu}\right)_{j j} \frac{x-a_{j}}{x}\left[-2+\frac{x-a_{j}+2 a_{h}}{x-a_{j}} \ln \left(\frac{x-a_{j}+a_{h}}{a_{h}}\right)\right], \\
\hat{\sigma}_{t_{j}}^{(7)} & =3 \alpha_{u}\left(\lambda_{\nu}^{\dagger} \lambda_{\nu}\right)_{j j}\left[-\frac{x-a_{j}}{x-a_{j}+2 a_{h}}+\ln \left(\frac{x-a_{j}+a_{h}}{a_{h}}\right)\right], \\
\hat{\sigma}_{t_{j}}^{(8)} & =3 \alpha_{u}\left(\lambda_{\nu}^{\dagger} \lambda_{\nu}\right)_{j j} \frac{x-a_{j}}{x} \frac{a_{j}}{x} \\
\hat{\sigma}_{t_{j}}^{(9)} & =3 \alpha_{u}\left(\lambda_{\nu}^{\dagger} \lambda_{\nu}\right)_{j j} \frac{a_{j}}{x}\left[-\frac{x-a_{j}}{x-a_{j}+a_{h}}+\ln \left(\frac{x-a_{j}+a_{h}}{a_{h}}\right)\right] .
\end{aligned}
$$

\section{E.3 Neutrino Pair Creation and Annihilation}

With the abbreviations

$$
\begin{aligned}
\lambda_{i j} & =\lambda\left(x, a_{i}, a_{j}\right)=\left[x-\left(\sqrt{a_{i}}+\sqrt{a_{j}}\right)^{2}\right]\left[x-\left(\sqrt{a_{i}}-\sqrt{a_{j}}\right)^{2}\right] \\
\mathrm{L}_{i j} & =\ln \left(\frac{x-a_{i}-a_{j}+\sqrt{\lambda_{i j}}}{x-a_{i}-a_{j}-\sqrt{\lambda_{i j}}}\right)
\end{aligned}
$$

the reduced cross sections for the right-handed neutrino pair creation read

$$
\hat{\sigma}_{N_{i} N_{j}}^{(1)}=\frac{1}{4 \pi}\left\{\left(\lambda_{\nu}^{\dagger} \lambda_{\nu}\right)_{j j}\left(\lambda_{\nu}^{\dagger} \lambda_{\nu}\right)_{i i}\left[-\frac{2}{x} \sqrt{\lambda_{i j}}+\mathrm{L}_{i j}\right]-2 \operatorname{Re}\left[\left(\lambda_{\nu}^{\dagger} \lambda_{\nu}\right)_{j i}^{2}\right] \frac{\sqrt{a_{i} a_{j}}\left(a_{i}+a_{j}\right)}{x\left(x-a_{i}-a_{j}\right)} \mathrm{L}_{i j}\right\},
$$




$$
\begin{aligned}
& \hat{\sigma}_{N_{i} N_{j}}^{(2)}=\frac{1}{4 \pi}\left\{\left(\lambda_{\nu}^{\dagger} \lambda_{\nu}\right)_{j j}\left(\lambda_{\nu}^{\dagger} \lambda_{\nu}\right)_{i i}\left[\frac{2}{x} \sqrt{\lambda_{i j}}+\frac{a_{i}+a_{j}}{x} \mathrm{~L}_{i j}\right]-2 \operatorname{Re}\left[\left(\lambda_{\nu}^{\dagger} \lambda_{\nu}\right)_{j i}^{2}\right] \frac{\sqrt{a_{i} a_{j}}}{x-a_{i}-a_{j}} \mathrm{~L}_{i j}\right\}, \\
& \hat{\sigma}_{N_{i} N_{j}}^{(3)}=\frac{1}{4 \pi}\left\{\left|\left(\lambda_{\nu}^{\dagger} \lambda_{\nu}\right)_{j i}\right|^{2}\left[-\frac{2}{x} \sqrt{\lambda_{i j}}+\mathrm{L}_{i j}\right]-2 \operatorname{Re}\left[\left(\lambda_{\nu}^{\dagger} \lambda_{\nu}\right)_{j i}^{2}\right] \frac{\sqrt{a_{i} a_{j}}\left(a_{i}+a_{j}\right)}{x\left(x-a_{i}-a_{j}\right)} \mathrm{L}_{i j}\right\}, \quad(\mathrm{E} .36) \\
& \hat{\sigma}_{N_{i} N_{j}}^{(4)}=\frac{1}{4 \pi}\left\{\left|\left(\lambda_{\nu}^{\dagger} \lambda_{\nu}\right)_{j i}\right|^{2}\left[\frac{2}{x} \sqrt{\lambda_{i j}}+\frac{a_{i}+a_{j}}{x} \mathrm{~L}_{i j}\right]-2 \operatorname{Re}\left[\left(\lambda_{\nu}^{\dagger} \lambda_{\nu}\right)_{j i}^{2}\right] \frac{\sqrt{a_{i} a_{j}}}{x-a_{i}-a_{j}} \mathrm{~L}_{i j}\right\} .
\end{aligned}
$$

For the scalar neutrinos one has similarly

$$
\begin{aligned}
& \hat{\sigma}_{\widetilde{N_{i}^{c}}(1)}^{\left(N_{j}^{c}\right.}=\frac{1}{4 \pi}\left(\lambda_{\nu}^{\dagger} \lambda_{\nu}\right)_{j j}\left(\lambda_{\nu}^{\dagger} \lambda_{\nu}\right)_{i i}\left[-\frac{2}{x} \sqrt{\lambda_{i j}}+\frac{x-a_{i}-a_{j}}{x} \mathrm{~L}_{i j}\right] \\
& \hat{\sigma}_{\bar{N}_{i}^{c} \widetilde{N}_{j}^{c}}^{(2)}=\frac{1}{4 \pi}\left\{\left(\lambda_{\nu}^{\dagger} \lambda_{\nu}\right)_{j j}\left(\lambda_{\nu}^{\dagger} \lambda_{\nu}\right)_{i i} \frac{2}{x} \sqrt{\lambda_{i j}}-2 \operatorname{Re}\left[\left(\lambda_{\nu}^{\dagger} \lambda_{\nu}\right)_{j i}^{2}\right] \frac{\sqrt{a_{i} a_{j}}}{x} \mathrm{~L}_{i j}\right\}, \\
& \hat{\sigma}_{\widetilde{N}_{i}^{c} \widetilde{N}_{j}^{c}}^{(3)}=\frac{1}{4 \pi}\left|\left(\lambda_{\nu}^{\dagger} \lambda_{\nu}\right)_{j i}\right|^{2}\left[-\frac{2}{x} \sqrt{\lambda_{i j}}+\frac{x-a_{i}-a_{j}}{x} \mathrm{~L}_{i j}\right] \\
& \hat{\sigma}_{\bar{N}_{i}^{c} \widetilde{N}_{j}^{c}}^{(4)}=\frac{1}{4 \pi}\left\{\left|\left(\lambda_{\nu}^{\dagger} \lambda_{\nu}\right)_{j i}\right|^{2} \frac{2}{x} \sqrt{\lambda_{i j}}-2 \operatorname{Re}\left[\left(\lambda_{\nu}^{\dagger} \lambda_{\nu}\right)_{j i}^{2}\right] \frac{\sqrt{a_{i} a_{j}}}{x} \mathrm{~L}_{i j}\right\} .
\end{aligned}
$$

For the diagrams involving one neutrino and one sneutrino (cf. fig. 3.14) one finally has

$$
\begin{aligned}
& \hat{\sigma}_{N_{j} \widetilde{N_{i}^{c}}}^{(1)}=\frac{1}{4 \pi}\left\{\left(\lambda_{\nu}^{\dagger} \lambda_{\nu}\right)_{j j}\left(\lambda_{\nu}^{\dagger} \lambda_{\nu}\right)_{i i} \frac{x+a_{i}-a_{j}}{x} \mathrm{~L}_{i j}-2 \operatorname{Re}\left[\left(\lambda_{\nu}^{\dagger} \lambda_{\nu}\right)_{j i}^{2}\right] \frac{\sqrt{a_{i} a_{j}}}{x} \frac{x+a_{i}-a_{j}}{x-a_{i}-a_{j}} \mathrm{~L}_{i j}\right\}, \\
& \hat{\sigma}_{N_{j} \widetilde{N_{i}^{c}}}^{(2)}=\frac{1}{4 \pi}\left\{\left|\left(\lambda_{\nu}^{\dagger} \lambda_{\nu}\right)_{j i}\right|^{2} \frac{x+a_{i}-a_{j}}{x} \mathrm{~L}_{i j}-2 \operatorname{Re}\left[\left(\lambda_{\nu}^{\dagger} \lambda_{\nu}\right)_{j i}^{2}\right] \frac{\sqrt{a_{i} a_{j}}}{x} \frac{x+a_{i}-a_{j}}{x-a_{i}-a_{j}} \mathrm{~L}_{i j}\right\} .
\end{aligned}
$$




\section{Appendix $\mathrm{F}$}

\section{Reaction Densities}

In general the reaction densities corresponding to the reduced cross sections discussed in appendix $\mathbb{E}$ have to be calculated numerically. However, there exist some interesting limiting cases where one can calculate them analytically. Since thermal averaging of reduced cross sections via eq. (D.16) involves modified Bessel functions, we start by summarizing a few useful formulae for Bessel functions before discussing the reaction densities.

\section{F.1 Bessel functions}

Modified Bessel functions with different indices are related via recursion relations [53],

$$
\begin{aligned}
& x \mathrm{~K}_{\nu-1}(x)+x \mathrm{~K}_{\nu+1}(x)=2 \nu \mathrm{K}_{\nu}(x), \\
& \mathrm{K}_{\nu-1}(x)-\mathrm{K}_{\nu+1}(x)=2 \frac{d}{d x} \mathrm{~K}_{\nu}(x) .
\end{aligned}
$$

For integer index Bessel functions have the following series representation

$$
\begin{aligned}
\mathrm{K}_{n}(x)= & \frac{1}{2} \sum_{k=0}^{n-1}(-1)^{k} \frac{(n-k-1) !}{k !\left(\frac{z}{2}\right)^{n-2 k}}+ \\
& +(-1)^{n+1} \sum_{k=0}^{\infty} \frac{\left(\frac{z}{2}\right)^{n+2 k}}{k !(n+k) !}\left[\ln \left(\frac{x}{2}\right)-\frac{1}{2} \psi(k+1)-\frac{1}{2} \psi(n+k+1)\right]
\end{aligned}
$$

where $\psi$ denotes the derivative of the logarithm of the Gamma function

$$
\psi(x)=\frac{d}{d x} \ln \Gamma(x) .
$$


For integer argument it reads

$$
\psi(n)=-\gamma_{\mathrm{E}}+\sum_{k=1}^{n-1} \frac{1}{k},
$$

where $\gamma_{\mathrm{E}}=0.577216$ is Euler's constant. Hence, the leading terms of the series are given by

$$
\begin{aligned}
& \mathrm{K}_{0}(x)=\ln \left(\frac{2}{x}\right)-\gamma_{\mathrm{E}}+\ldots, \\
& \mathrm{K}_{n}(x)=\frac{(n-1) !}{2}\left(\frac{2}{x}\right)^{n}+\ldots, \quad \text { for } n \geq 1 .
\end{aligned}
$$

The asymptotic expansion of modified Bessel functions reads

$$
\mathrm{K}_{\nu}(x)=\sqrt{\frac{\pi}{2 x}} \mathrm{e}^{-x} \sum_{k=0}^{\infty} \frac{1}{k !(2 x)^{k}} \frac{\Gamma\left(\nu+k+\frac{1}{2}\right)}{\Gamma\left(\nu-k+\frac{1}{2}\right)},
$$

i.e. to leading order all Bessel functions have the same asymptotic behaviour,

$$
\mathrm{K}_{\nu}(x)=\sqrt{\frac{\pi}{2 x}} \mathrm{e}^{-x}+\ldots
$$

Furthermore, when evaluating reaction densities according to eq. (D.16), one has to compute integrals involving Bessel functions. In the following we compile the integrals that we have used in our calculations.

\section{Bessel functions and powers}

$$
\begin{aligned}
& \int_{0}^{\infty} d x x^{\mu} \mathrm{K}_{\nu}(a x)=2^{\mu-1} a^{-\mu-1} \Gamma\left(\frac{1+\mu+\nu}{2}\right) \Gamma\left(\frac{1+\mu-\nu}{2}\right) \\
& \text { for } \operatorname{Re}(1+\mu \pm \nu)>0 \text { and } \operatorname{Re}(a)>0 \\
& \int_{0}^{1} d x x^{\nu+1} \mathrm{~K}_{\nu}(a x)=2^{\nu} a^{-\nu-2} \Gamma(\nu+1)-\frac{1}{a} \mathrm{~K}_{\nu+1}(a) \quad \text { for } \operatorname{Re}(\nu)>-1, \\
& \int_{0}^{1} d x x^{1-\nu} \mathrm{K}_{\nu}(a x)=2^{-\nu} a^{\nu-2} \Gamma(1-\nu)-\frac{1}{a} \mathrm{~K}_{\nu-1}(a) \quad \text { for } \operatorname{Re}(\nu)<1, \\
& \int_{a}^{\infty} d x \mathrm{~K}_{1}(z x)=\frac{1}{z} \mathrm{~K}_{0}(z a) \\
& \int_{a}^{\infty} d x x^{2} \mathrm{~K}_{1}(z x)=\frac{a^{2}}{z} \mathrm{~K}_{2}(z a)
\end{aligned}
$$




$$
\begin{aligned}
& \int_{0}^{\infty} d x x^{2} \mathrm{~K}_{1}(z x)=\frac{2}{z^{3}}, \\
& \int_{a}^{\infty} d x x \mathrm{~K}_{0}(z x)=\frac{a}{z} \mathrm{~K}_{1}(z a), \\
& \int_{0}^{\infty} d x x \mathrm{~K}_{0}(z x)=\frac{1}{z^{2}}, \\
& \int_{a}^{\infty} d x \sqrt{x} \mathrm{~K}_{1}(z \sqrt{x})=2 \frac{a}{z} \mathrm{~K}_{2}(z \sqrt{a}), \\
& \int_{0}^{\infty} d x \sqrt{x} \mathrm{~K}_{1}(z \sqrt{x})=\frac{4}{z^{3}}, \\
& \int_{a}^{\infty} d x \frac{1}{\sqrt{x}} \mathrm{~K}_{1}(z \sqrt{x})=\frac{2}{z} \mathrm{~K}_{0}(z \sqrt{a}) .
\end{aligned}
$$

\section{Bessel functions and logarithms}

$$
\begin{aligned}
& \int_{a}^{\infty} d x x \ln (x) \mathrm{K}_{0}(z x)=\frac{1}{z^{2}} \mathrm{~K}_{0}(z a)+a \ln (a) \frac{1}{z} \mathrm{~K}_{1}(z a), \\
& \int_{0}^{\infty} d x x \ln (x) \mathrm{K}_{0}(z x)=\frac{1}{z^{2}}\left[-\gamma_{\mathrm{E}}+\ln \left(\frac{2}{z}\right)\right], \\
& \int_{a}^{\infty} d x \sqrt{x} \ln \left(\frac{x}{b}\right) \mathrm{K}_{1}(z \sqrt{x})=\left[\frac{8}{z^{3}}+\frac{2 a}{z} \ln \left(\frac{a}{b}\right)\right] \mathrm{K}_{0}(z \sqrt{a}) \\
& \quad+4 \frac{\sqrt{a}}{z^{2}}\left[1+\ln \left(\frac{a}{b}\right)\right] \mathrm{K}_{1}(z \sqrt{a}), \\
& \int_{0}^{\infty} d x \sqrt{x} \ln \left(\frac{x}{b}\right) \mathrm{K}_{1}(z \sqrt{x})=\frac{4}{z^{3}}\left[1-2 \gamma_{\mathrm{E}}+\ln \left(\frac{4}{b z^{2}}\right)\right] .
\end{aligned}
$$

In all these integrals we have always assumed that $a, b$ and $z$ are real and positive. 


\section{F.2 Lepton Number Violating Scatterings}

In the Boltzmann equations we do not need every reaction density $\gamma_{N}^{(i)}, i=1, \ldots, 19$ separately (cf. sect. 4.1). We only have to consider the combined reaction densities

$$
\begin{aligned}
& \gamma_{A}^{\Delta L}=2 \gamma_{N}^{(1)}+\gamma_{N}^{(3)}+\gamma_{N}^{(4)}+\gamma_{N}^{(6)}+\gamma_{N}^{(7)}+2 \gamma_{N}^{(12)}+\gamma_{N}^{(14)}, \\
& \gamma_{B}^{\Delta L}=\gamma_{N}^{(3)}+\gamma_{N}^{(4)}-\gamma_{N}^{(6)}-\gamma_{N}^{(7)}+\gamma_{N}^{(14)}, \\
& \gamma_{C}^{\Delta L}=3 \gamma_{N}^{(9)}+\gamma_{N}^{(17)}+\gamma_{N}^{(18)}+6 \gamma_{N}^{(19)}, \\
& \gamma_{D}^{\Delta L}=4 \gamma_{N}^{(5)}+2 \gamma_{N}^{(8)}+8 \gamma_{N}^{(10)}+3 \gamma_{N}^{(9)}+4 \gamma_{N}^{(15)}+2 \gamma_{N}^{(16)}+\gamma_{N}^{(17)}+\gamma_{N}^{(18)}+6 \gamma_{N}^{(19)} .
\end{aligned}
$$

For low temperatures, i.e. $z \gg 1 / \sqrt{a_{j}}$, the dominant contribution to the integrand of the reaction densities comes from small centre of mass energies, i.e. $x \ll a_{j}$. In this limit the reduced cross sections $\hat{\sigma}_{N}^{(i)}$ for the $(L+\widetilde{L})$ violating or conserving processes behave differently. For the $(L+\widetilde{L})$ violating scatterings $(i=1, \ldots, 5,8,10,12, \ldots, 16)$ one finds

$$
\hat{\sigma}_{N}^{(i)} \propto x \quad \text { for } x \ll a_{j}
$$

while one has

$$
\hat{\sigma}_{N}^{(i)} \propto x^{2} \quad \text { for } x \ll a_{j}
$$

for the $(L+\widetilde{L})$ conserving processes $(i=6,7,9,11,17,18,19)$. In diagrams with an intermediate neutrino this different behaviour is due to the different chiral parts of the fermionic propagator contributing to the scatterings. $(L+\widetilde{L})$ violating processes contain the chirality violating propagators $\sqrt{a_{j}} /\left(x-a_{j}\right)$, whereas $(L+\widetilde{L})$ conserving processes depend on the chirality conserving propagator $\sqrt{x} /\left(x-a_{j}\right)$. For diagrams with an intermediate scalar neutrino the corresponding kinematical factors originate in the couplings of sneutrinos to different initial and final states.

Hence, the reaction densities can be calculated analytically in this limit and one finds

$$
\begin{aligned}
& \gamma_{A}^{\Delta L}=\frac{M_{1}^{4}}{\pi^{5}} \frac{1}{z^{6}}\left\{\sum_{j}\left(\lambda_{\nu}^{\dagger} \lambda_{\nu}\right)_{j j}^{2} \frac{2}{a_{j}}+\sum_{\substack{n, j \\
j<n}} \operatorname{Re}\left[\left(\lambda_{\nu}^{\dagger} \lambda_{\nu}\right)_{n j}^{2}\right] \frac{19}{4 \sqrt{a_{n} a_{j}}}\right\}, \\
& \gamma_{B}^{\Delta L}=\frac{M_{1}^{4}}{\pi^{5}} \frac{1}{z^{6}}\left\{\sum_{j}\left(\lambda_{\nu}^{\dagger} \lambda_{\nu}\right)_{j j}^{2} \frac{1}{2 a_{j}}+\sum_{\substack{n, j \\
j<n}} \operatorname{Re}\left[\left(\lambda_{\nu}^{\dagger} \lambda_{\nu}\right)_{n j}^{2}\right] \frac{7}{4 \sqrt{a_{n} a_{j}}}\right\}, \\
& \gamma_{C}^{\Delta L}=\frac{M_{1}^{4}}{\pi^{5}} \frac{1}{z^{8}}\left\{\sum_{j}\left(\lambda_{\nu}^{\dagger} \lambda_{\nu}\right)_{j j}^{2} \frac{1}{a_{j}^{2}}\left(4+\frac{27 \alpha_{u}}{4 \pi}\right)+\sum_{\substack{n, j \\
j<n}}\left|\left(\lambda_{\nu}^{\dagger} \lambda_{\nu}\right)_{n j}\right|^{2} \frac{1}{a_{j} a_{n}}\left(8+\frac{18 \alpha_{u}}{\pi}\right)\right\},
\end{aligned}
$$




$$
\gamma_{D}^{\Delta L}=\frac{M_{1}^{4}}{\pi^{5}} \frac{\alpha_{u}}{\pi} \frac{1}{z^{6}}\left\{\sum_{j}\left(\lambda_{\nu}^{\dagger} \lambda_{\nu}\right)_{j j}^{2} \frac{153}{32 a_{j}}+\sum_{\substack{n, j \\ j<n}} \operatorname{Re}\left[\left(\lambda_{\nu}^{\dagger} \lambda_{\nu}\right)_{n j}^{2}\right] \frac{147}{16 \sqrt{a_{n} a_{j}}}\right\}
$$

For high temperatures, i.e. $z \ll 1 / \sqrt{a_{j}}$, we can use the asymptotic expansions of the reduced cross sections to compute the reactions densities and we get

$$
\begin{aligned}
& \gamma_{A}^{\Delta L}=\frac{M_{1}^{4}}{64 \pi^{5}} \frac{1}{z^{4}}\left\{\left(13+\frac{3 \alpha_{u}}{4 \pi}\right) \sum_{j}\left(\lambda_{\nu}^{\dagger} \lambda_{\nu}\right)_{j j}^{2}+\sum_{\substack{n, j \\
j<n}} \operatorname{Re}\left[\left(\lambda_{\nu}^{\dagger} \lambda_{\nu}\right)_{n j}^{2}\right] \frac{24 \sqrt{a_{n} a_{j}}}{a_{n}-a_{j}} \ln \left(\frac{a_{n}}{a_{j}}\right)\right. \\
& \left.+\left(2+\frac{3 \alpha_{u}}{2 \pi}\right) \sum_{\substack{n, j \\
j<n}}\left|\left(\lambda_{\nu}^{\dagger} \lambda_{\nu}\right)_{n j}\right|^{2}\right\} \\
& \gamma_{B}^{\Delta L}=\frac{M_{1}^{4}}{64 \pi^{5}} \frac{1}{z^{4}}\left\{\left(3-\frac{3 \alpha_{u}}{4 \pi}\right) \sum_{j}\left(\lambda_{\nu}^{\dagger} \lambda_{\nu}\right)_{j j}^{2}+\sum_{\substack{n, j \\
j<n}} \operatorname{Re}\left[\left(\lambda_{\nu}^{\dagger} \lambda_{\nu}\right)_{n j}^{2}\right] \frac{8 \sqrt{a_{n} a_{j}}}{a_{n}-a_{j}} \ln \left(\frac{a_{n}}{a_{j}}\right)\right. \\
& \left.-\left(2+\frac{3 \alpha_{u}}{2 \pi}\right) \sum_{\substack{n, j \\
j<n}}\left|\left(\lambda_{\nu}^{\dagger} \lambda_{\nu}\right)_{n j}\right|^{2}\right\} \\
& \gamma_{C}^{\Delta L}=\frac{M_{1}^{4}}{32 \pi^{5}} \frac{1}{z^{4}}\left\{\sum_{j}\left(\lambda_{\nu}^{\dagger} \lambda_{\nu}\right)_{j j}^{2}\left[-1-\frac{45 \alpha_{u}}{8 \pi}+\frac{9 \alpha_{u}}{8} \sqrt{\frac{a_{j}}{\widetilde{c_{j}}}}+\left(4+\frac{27 \alpha_{u}}{2 \pi}\right)\left(\ln \left(\frac{2}{z \sqrt{a_{j}}}\right)-\gamma_{\mathrm{E}}\right)\right]\right. \\
& +\sum_{\substack{n, j \\
j<n}}\left|\left(\lambda_{\nu}^{\dagger} \lambda_{\nu}\right)_{n j}\right|^{2}\left[2+\frac{9 \alpha_{u}}{\left(a_{n}-a_{j}\right)^{2}}\left(a_{n} \sqrt{a_{j} \widetilde{c_{j}}}+a_{j} \sqrt{a_{n} \widetilde{c_{n}}}\right)\right. \\
& \left.\left.+\left(8+\frac{36 \alpha_{u}}{\pi}\right)\left(\frac{a_{n}}{a_{n}-a_{j}} \ln \left(\frac{2}{\sqrt{a_{n}} z}\right)+\frac{a_{j}}{a_{j}-a_{n}} \ln \left(\frac{2}{\sqrt{a_{j}} z}\right)-\gamma_{\mathrm{E}}\right)\right]\right\}, \\
& \gamma_{D}^{\Delta L}=\frac{M_{1}^{4}}{32 \pi^{5}} \frac{1}{z^{4}}\left\{\sum_{j}\left(\lambda_{\nu}^{\dagger} \lambda_{\nu}\right)_{j j}^{2}\left[-1+\frac{27 \alpha_{u}}{8 \pi}+\frac{39 \alpha_{u}}{8} \sqrt{\frac{a_{j}}{\widetilde{c_{j}}}}+\left(4+\frac{27 \alpha_{u}}{2 \pi}\right)\left(\ln \left(\frac{2}{z \sqrt{a_{j}}}\right)-\gamma_{\mathrm{E}}\right)\right]\right. \\
& +\sum_{\substack{n, j \\
j<n}}\left|\left(\lambda_{\nu}^{\dagger} \lambda_{\nu}\right)_{n j}\right|^{2}\left[4-8 \gamma_{\mathrm{E}}+\frac{8}{a_{n}-a_{j}}\left(a_{n} \ln \left(\frac{2}{\sqrt{a_{n}} z}\right)-a_{j} \ln \left(\frac{2}{\sqrt{a_{j}} z}\right)\right)\right] \\
& \left.+\frac{3 \alpha_{u}}{\pi} \sum_{\substack{n, j \\
j<n}} \operatorname{Re}\left[\left(\lambda_{\nu}^{\dagger} \lambda_{\nu}\right)_{n j}^{2}\right] \sqrt{a_{n} a_{j}}\left[\frac{7}{2} \frac{1}{a_{n}-a_{j}} \ln \left(\frac{a_{n}}{a_{j}}\right)+\frac{5 \pi}{\left(a_{n}-a_{j}\right)^{2}}\left(\sqrt{a_{j} \widetilde{c_{j}}}+\sqrt{a_{n} \widetilde{c_{n}}}\right)\right]\right\},
\end{aligned}
$$

i.e. these reaction densities are proportional to $T^{4}$ at high temperatures, as expected on purely 
dimensional grounds.

For intermediate temperatures $z \sim 1 / \sqrt{a_{j}}$ the reaction densities have to be computed numerically. This becomes increasingly difficult in the narrow width limit, where $1 / D_{j}(x)$ has two very sharp peaks. However, in the limit $c_{j} \rightarrow 0$ the two peaks in $1 / D_{j}(x)$ cancel each other, since they have a different sign, while the peaks in $1 / D_{j}^{2}(x)$ add up. Therefore, the terms proportional to $1 / D_{j}(x)$ or $1 / D_{j}(x) D_{n}(x)$ with $n \neq j$ can be neglected in the narrow width limit, while $1 / D_{j}^{2}(x)$ can be approximated by a $\delta$-function

$$
\frac{1}{D_{j}^{2}(x)} \approx \frac{\pi}{2 \sqrt{a_{j} c_{j}}} \delta\left(x-a_{j}\right) .
$$

An analogous relation holds for $1 /{\widetilde{D_{j}}}^{2}(x)$.

These relations allow to calculate the contributions from the $s$-channel diagrams to the reaction densities analytically in the limit $c_{j} \rightarrow 0$, while the contributions from the $t$-channel diagrams can easily be evaluated numerically.

\section{F.3 Stop Neutrino Scatterings}

The reaction densities $\gamma_{t_{j}}^{(i)}$ for the interaction of a (s)neutrino with a top or a stop can also be calculated analytically in the limit of high temperatures $z \ll 1 / \sqrt{a_{j}}$. For the $s$-channel processes one finds

$$
\begin{aligned}
& \gamma_{t_{j}}^{(0)}=\frac{3 \alpha_{u} M_{1}^{4}}{64 \pi^{4}}\left(\lambda_{\nu}^{\dagger} \lambda_{\nu}\right)_{j j} a_{j} \frac{\mathrm{K}_{2}\left(z \sqrt{a_{j}}\right)}{z^{2}} \\
& \gamma_{t_{j}}^{(3)}=2 \gamma_{t_{j}}^{(0)}, \quad \gamma_{t_{j}}^{(5)}=\gamma_{t_{j}}^{(0)}
\end{aligned}
$$

For the $t$-channel reaction densities one has analogously

$$
\begin{gathered}
\gamma_{t_{j}}^{(1)}=\frac{3 \alpha_{u} M_{1}^{4}}{8 \pi^{4}}\left(\lambda_{\nu}^{\dagger} \lambda_{\nu}\right)_{j j} \frac{1}{z^{4}}\left[\left(1-\frac{z^{2} a_{j}}{4}\right) \mathrm{K}_{0}\left(z \sqrt{a_{j}}\right)+\frac{z^{2} a_{j}}{4}\left(\ln \left(\frac{a_{j}}{a_{h}}\right)-1\right) \mathrm{K}_{2}\left(z \sqrt{a_{j}}\right)\right], \\
\gamma_{t_{j}}^{(2)}=\frac{3 \alpha_{u} M_{1}^{4}}{8 \pi^{4}}\left(\lambda_{\nu}^{\dagger} \lambda_{\nu}\right)_{j j} \frac{1}{z^{4}}\left[\left(1-\frac{z^{2} a_{j}}{4}\right) \mathrm{K}_{0}\left(z \sqrt{a_{j}}\right)+\frac{z^{2} a_{j}}{4} \ln \left(\frac{a_{j}}{a_{h}}\right) \mathrm{K}_{2}\left(z \sqrt{a_{j}}\right)\right], \\
\gamma_{t_{j}}^{(4)}=2 \gamma_{t_{j}}^{(0)}, \quad \gamma_{t_{j}}^{(6)}=\gamma_{t_{j}}^{(1)}, \quad \gamma_{t_{j}}^{(7)}=\gamma_{t_{j}}^{(2)} .
\end{gathered}
$$

$\gamma_{t_{j}}^{(8)}$ and $\gamma_{t_{j}}^{(9)}$ are several orders of magnitude smaller than the other $\gamma_{t_{j}}^{(i)}$ for small $z$ and can therefore be neglected at high temperatures.

By using the series expansions (F.6) and (F.7), one sees that the processes with a higgsino in the $t$-channel, i.e. $\gamma_{t_{j}}^{(1)}, \gamma_{t_{j}}^{(2)}, \gamma_{t_{j}}^{(6)}$ and $\gamma_{t_{j}}^{(7)}$, behave like $T^{4} \ln \left(T / M_{j}\right)$ at high temperatures, whereas the other reaction densities are proportional to $T^{4}$. 


\section{F.4 Pair Creation and Annihilation of Neutrinos}

In the Boltzmann equations we only need certain combinations of reaction densities which can easily be evaluated for high temperatures

$$
\begin{aligned}
\sum_{k=1}^{2} \gamma_{N_{i} N_{j}}^{(k)}= & \sum_{k=1}^{2} \gamma_{\widetilde{N_{i}^{c}} \widetilde{N_{j}^{c}}}^{(k)}=\gamma_{N_{j} \widetilde{N}_{i}^{c}}^{(1)}= \\
= & \frac{M_{1}^{4}}{16 \pi^{5}} \frac{1}{z^{4}}\left(\lambda_{\nu}^{\dagger} \lambda_{\nu}\right)_{j j}\left(\lambda_{\nu}^{\dagger} \lambda_{\nu}\right)_{i i}\left\{\left[1-\frac{z^{2}}{4}\left(\sqrt{a_{i}}+\sqrt{a_{j}}\right)^{2}\right] \mathrm{K}_{0}\left(z\left(\sqrt{a_{i}}+\sqrt{a_{j}}\right)\right)\right. \\
& \left.+\frac{z^{2}}{4}\left(\sqrt{a_{i}}+\sqrt{a_{j}}\right)^{2}\left[1+\ln \left(2+\frac{a_{i}+a_{j}}{\sqrt{a_{i} a_{j}}}\right)\right] \mathrm{K}_{2}\left(z\left(\sqrt{a_{i}}+\sqrt{a_{j}}\right)\right)\right\} \\
\sum_{k=3}^{4} \gamma_{N_{i} N_{j}}^{(k)}= & \sum_{k=3}^{4} \gamma_{\widetilde{N_{i}^{c}} \widetilde{N}_{j}^{c}}^{(k)}=\gamma_{N_{j} \widetilde{N}_{i}^{c}}^{(2)}= \\
& \frac{M_{1}^{4}}{16 \pi^{5}} \frac{1}{z^{4}}\left|\left(\lambda_{\nu}^{\dagger} \lambda_{\nu}\right)_{j i}\right|^{2}\left\{\left[1-\frac{z^{2}}{4}\left(\sqrt{a_{i}}+\sqrt{a_{j}}\right)^{2}\right] \mathrm{K}_{0}\left(z\left(\sqrt{a_{i}}+\sqrt{a_{j}}\right)\right)\right. \\
& \left.+\frac{z^{2}}{4}\left(\sqrt{a_{i}}+\sqrt{a_{j}}\right)^{2}\left[1+\ln \left(2+\frac{a_{i}+a_{j}}{\sqrt{a_{i} a_{j}}}\right)\right] \mathrm{K}_{2}\left(z\left(\sqrt{a_{i}}+\sqrt{a_{j}}\right)\right)\right\}
\end{aligned}
$$

i.e. these reaction densities are proportional to $T^{4} \ln \left(T /\left(M_{i}+M_{j}\right)\right)$ at high temperatures. 


\section{Bibliography}

[1] E. E. Cummings, 73 poems, (Faber and Faber, London, 1990)

[2] For a discussion and references, see E. W. Kolb and M. S. Turner, The Early Universe, (Addison-Wesley, Redwood City, CA, 1990)

[3] A. D. Sakharov, JETP Letters 5 (1967) 24

[4] For a recent review, see R. H. Brandenberger, Particle Physics Aspects of Modern Cosmology, to be published in Field Theoretical Methods in Fundamental Physics, ed. by Choonkyu Lee, Mineumsa Co. Ltd., Seoul, 1997, Preprint BROWN-HET-1067, hep-ph/9701276

[5] L. Kofman, A. Linde and A. A. Starobinsky, Phys. Rev. D 56 (1997) 3258

I. Zlatev, G. Huey and P. Steinhardt, Phys. Rev. D 57 (1998) 2152

[6] I. Affleck and M. Dine, Nucl. Phys. B 249 (1985) 361

[7] see, e.g., L. Randall, in Electroweak Interactions and Unified Theories, Proc. of the XXXth Rencontres de Moriond, ed. J. Trân Thanh Vân, (Editions Frontières, 1995)

[8] For a review and references, see V. A. Rubakov and M. E. Shaposhnikov, Phys. Usp. 39 (1996) $461 ;$

[9] For a discussion and references, see K. Jansen, Nucl. Phys. B (Proc. Supp.) 47 (1996) 196

[10] V. A. Kuzmin, V. A. Rubakov and M. E. Shaposhnikov, Phys. Lett. B 155 (1985) 36

[11] T. Yanagida, in: Workshop on unified Theories, KEK report 79-18 (1979) p. 95;

M. Gell-Mann et al., in: Supergravity, Proc. of the Stony Brook Workshop 1979, eds. P. van Nieuwenhuizen and D. Freedman (North Holland, Amsterdam, 1979), p. 315

[12] M. Fukugita and T. Yanagida, Phys. Lett. B 174 (1986) 45 
[13] M. A. Luty, Phys. Rev. D 45 (1992) 455

[14] M. Plümacher, Z. Phys. C 74 (1997) 549

[15] W. Buchmüller and M. Plümacher, Phys. Lett. B 389 (1996) 73

[16] B. A. Campbell, S. Davidson and K. A. Olive, Nucl. Phys. B 399 (1993) 111

[17] M. Plümacher, hep-ph/9704231, to appear in Nucl. Phys. B

[18] L. Covi, E. Roulet and F. Vissani, Phys. Lett. B 384 (1996) 169

[19] A. Yu. Ignatev,V. A. Kuzmin and M. E. Shaposhnikov, JETP Lett. 30 (1979) 688;

F. J. Botella and J. Roldan, Phys. Rev. D 44 (1991) 966;

J. Liu and G. Segrè, Phys. Rev. D 48 (1993) 4609; Phys. Rev. D 49 (1994) 1342

[20] M. Flanz, E. A. Paschos and U. Sarkar, Phys. Lett. B 345 (1995) 248; ibid. B 384 (1996) 487 $(\mathrm{E})$;

L. Covi, N. Rius, E. Roulet and F. Vissani, Phys. Rev. D 57 (1998) 93

[21] M. Flanz, E. A. Paschos, U. Sarkar and J. Weiss, Phys. Lett. B 389 (1996) 693;

L. Covi and E. Roulet, Phys. Lett. B 399 (1997) 113;

A. Pilaftsis, Nucl. Phys. B 504 (1997) 61; Phys. Rev. D 56 (1997) 5431

[22] W. Buchmüller and M. Plümacher, Phys. Lett. B 431 (1998) 354

[23] M. Veltman, Physica 29 (1963) 186

[24] E. Roulet, L. Covi and F. Vissani, hep-ph/9712468

[25] S. J. Gates, M. T. Grisaru, M. Roček and W. Siegel, Superspace or One Thousand and One Lessons in Supersymmetry, (Benjamin/Cummings, Reading, MA, 1983)

[26] H. P. Nilles, Phys. Rep. 110 (1984) 1

[27] M. Sohnius, Phys. Rep. 128 (1985) 39

[28] A. Salam and J. Strathdee, Nucl. Phys. B 76 (1974) 477

[29] P. P. Srivastava, Supersymmetry, Superfields and Supergravity, (Adam Hilger, Bristol, 1986)

[30] J. Wess and J. Bagger, Supersymmetry and Supergravity, (Princeton University Press, Princeton, NJ, 1992) 
[31] B. Zumino, Nucl. Phys. B 89 (1975) 535

[32] M. T. Grisaru, W. Siegel and M. Roček, Nucl. Phys. B 159 (1979) 429

[33] J. Iliopoulos and B. Zumino, Nucl. Phys. B 76 (1974) 310

[34] E. Witten, Nucl. Phys. B 188 (1981) 513

[35] S. Dimopoulos and L. Susskind, Phys. Rev. D 18 (1978) 4500

[36] A. D. Dolgov and Ya. B. Zeldovich, Rev. Mod. Phys. 53 (1981) 1;

E. W. Kolb and S. Wolfram, Nucl. Phys. B 172 (1980) 224, ibid. B 195 (1982) 542 (E)

[37] W.-Y. Keung and L. Littenberg, Phys. Rev. D 28 (1983) 1067

[38] S. Yu. Khlebnikov and M. E. Shaposhnikov Nucl. Phys. B 308 (1988) 885

[39] L. E. Ibáñez and F. Quevedo, Phys. Lett. B 283 (1992) 261

[40] W. Buchmüller and D. Wyler, Phys. Lett. B 249 (1990) 458

[41] L. Wolfenstein, Phys. Rev. Lett. 51 (1983) 1945

[42] W. C. Haxton, Annu. Rev. Astron. Astrophys., 33 (1995) 459

[43] L. Wolfenstein, Phys. Rev. D 17 (1978) 2369;

S. P. Mikheyev and A. Y. Smirnov, Nuovo Cim. 9C (1986) 17

[44] E. Witten, Phys. Lett. B 91 (1980) 81;

Z. Berezhiani, in: High Energy Physics and Cosmology, Proc. of the 1995 ICTP Summer School, Trieste, eds. E. Gava, A. Masiero and K. S. Narain (World Scientific, Singapore, 1997), p. 618

[45] T. Moroi, Ph. D. Thesis, hep-ph/9503210

[46] Super-Kamiokande Collaboration, Y. Fukuda et al., hep-ex/9803006;

E. Kearns, Talk at News about SNUS, ITP Workshop, Santa Barbara, Dec. 1997, http://www.itp.ucsb.edu/online/snu/kearns/

[47] H. Murayama and T. Yanagida, Phys. Lett. B 322 (1994) 349;

M. Dine, L. Randall and S. Thomas, Nucl. Phys. B 458 (1996) 291;

G. Lazarides, R. K. Schaefer and Q. Shafi, Phys. Rev. D 56 (1997) 1324 
[48] B. A. Campbell, S. Davidson, J. Ellis and K. A. Olive, Phys. Lett. B 297 (1992) 118;

J. M. Cline, K. Kainulainen and K. A. Olive, Phys. Rev. D 49 (1994) 6394

[49] D. Boyanovsky, I. D. Lawrie and D.-S. Lee, Phys. Rev. D 54 (1996) 4013;

A. Riotto, hep-ph/9712221;

I. Joichi, Sh. Matsumoto and M. Yoshimura, hep-ph/9803201

[50] G. Passarino and M. Veltman, Nucl. Phys. B 160 (1979) 151

[51] A. Denner, Fortschr. Phys. 41 (1993) 307

[52] A. Denner, H. Eck, O. Hahn and J. Küblbeck, Phys. Lett. B 291 (1992) 278; Nucl. Phys. B 387 (1992) 467

[53] I. S. Gradstein and I. M. Ryshik, Tables of Series, Products and Integrals, (Harri Deutsch, Thun, 1981);

A. Erdélyi, W. Magnus, F. Oberhettinger and F. G. Tricomi, Higher Transcendental Functions, vol. II, (McGraw-Hill Book Company, 1953) 


\section{Acknowledgments}

I would like to thank my advisor, W. Buchmüller, who suggested this investigation, for continuous support and a close and fruitful collaboration.

I would also like to acknowledge highly instructive discussions with W. Beenakker, J. Hein, G. Hiller, A. Jakovác, S. Moch, T. Plehn and U. Nierste.

Finally, I would like to thank all my colleagues and friends at DESY for the enjoyable working atmosphere. 